\title{
Hemodialysis vascular access imaging : duplex ultrasound and contrast-enhanced magnetic resonance angiography
}

Citation for published version (APA):

Planken, R. N. (2007). Hemodialysis vascular access imaging : duplex ultrasound and contrast-enhanced magnetic resonance angiography. [Doctoral Thesis, Maastricht University]. Datawyse / Universitaire Pers Maastricht. https://doi.org/10.26481/dis.20070601rp

Document status and date:

Published: 01/01/2007

DOI:

10.26481/dis.20070601rp

Document Version:

Publisher's PDF, also known as Version of record

Please check the document version of this publication:

- A submitted manuscript is the version of the article upon submission and before peer-review. There can be important differences between the submitted version and the official published version of record.

People interested in the research are advised to contact the author for the final version of the publication, or visit the DOI to the publisher's website.

- The final author version and the galley proof are versions of the publication after peer review.

- The final published version features the final layout of the paper including the volume, issue and page numbers.

Link to publication

\footnotetext{
General rights rights.

- You may freely distribute the URL identifying the publication in the public portal. please follow below link for the End User Agreement:

www.umlib.nl/taverne-license

Take down policy

If you believe that this document breaches copyright please contact us at:

repository@maastrichtuniversity.nl

providing details and we will investigate your claim.
}

Copyright and moral rights for the publications made accessible in the public portal are retained by the authors and/or other copyright owners and it is a condition of accessing publications that users recognise and abide by the legal requirements associated with these

- Users may download and print one copy of any publication from the public portal for the purpose of private study or research.

- You may not further distribute the material or use it for any profit-making activity or commercial gain

If the publication is distributed under the terms of Article 25fa of the Dutch Copyright Act, indicated by the "Taverne" license above, 
Hemodialysis vascular access imaging

Duplex ultrasound and contrast-enhanced magnetic resonance angiography 
(C) Robrecht Nils Planken, Maastricht 2006

All rights reserved. No part of this book may be translated or reproduced in any form by photo, photoprint, microfilm or any other means without written permission from the author.

ISBN 978-90-5278-624-7

Cover illustration: Luca Antiga \& Robrecht Nils Planken

Streamlines representing flow patterns within a radio-cephalic arteriovenous fistula. The geometry was derived from an actual CE-MRA scan, while streamlines were generated using computational fluid dynamics.

Design and lay-out: Robrecht Nils Planken

Publisher: Datawyse, Universitaire Pers Maastricht 


\title{
Hemodialysis vascular access imaging
}

\author{
Duplex ultrasound and contrast-enhanced \\ magnetic resonance angiography
}

Proefschrift ter verkrijging van de graad van doctor aan de Universiteit Maastricht, op gezag van de Rector Magnificus Prof. Mr. G.P.M.F. Mols volgens het besluit van het College van Decanen, in het openbaar te verdedigen op vrijdag 1 juni 2007 om 12:00 uur, door Robrecht Nils Planken geboren op 17 februari 1978, te 's Gravenhage.

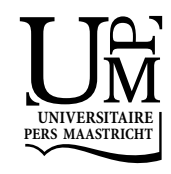


Promotores

Prof. Dr. P.J.E.H.M. Kitslaar

Prof. Dr. J.M.A. van Engelshoven

Co-promotores

Dr. J.H.M. Tordoir

Dr. T. Leiner

Beoordelingscommissie

Prof. Dr. Ir. A.P.G. Hoeks (voorzitter)

Dr. L.E.M. Duijm

Prof. Dr. K.M.L. Leunissen

Prof. Dr. Ir. F.N. van de Vosse

Financial support by the Dutch Kidney Foundation for the publication of this thesis is gratefully acknowledged.

Additional financial support was granted by: Philips Medical Systems, Vital Images, Edwards Lifesciences, Cordis a Johnson \& Johnson company, Fresenius Medical Care, Boston Scientific Benelux, WL Gore \& associates, Guerbet Nederland, Siemens Nederland, Toshiba Medical Systems Nederland, Baxter, Pie Medical Benelux, Transonic Systems Europe. 




\section{Contents}

Preface

Chapter 1 Introduction

Chapter 2 Current techniques for assessment of upper extremity vasculature prior to hemodialysis vascular access creation

Chapter 3 Forearm cephalic vein cross-sectional area changes at incremental congestion pressures: towards a standardized and reproducible vein mapping protocol

Chapter 4 Diameter measurements of the forearm cephalic vein prior to vascular access creation in end-stage renal disease patients: graduated pressure cuff versus tourniquet dilatation

Chapter 5 Magnetic resonance angiographic assessment of upper extremity vessels prior to vascular access creation: feasibility and accuracy

Chapter 6 Contrast-enhanced magnetic resonance angiography for prediction of hemodialysis vascular access early failure and nonmaturation: a prospective multi-center study

Chapter 7 Accessory veins and radial cephalic arteriovenous fistula nonmaturation: a prospective analysis using contrast-enhanced magnetic resonance angiography

Chapter 8 Stenosis detection in forearm hemodialysis arteriovenous fistulae by multiphase contrast-enhanced magnetic resonance angiography: preliminary experience

Chapter 9 General discussion, future perspectives and conclusions

Chapter 10 Samenvatting (summary in Dutch)

Curriculum vitae 

Preface 


\section{Preface}

The Dutch academic degree of Doctorandus (equivalent to a Master of Science degree) literally means: "He who should become a doctor". Starting my PhDproject was a conscious choice to take up the challenges of academia in an attempt to contribute to the rapidly evolving science. I was triggered by curiosity about the unknown and driven by ambition and conviction. I wanted to enter the world of science by obtaining the $\mathrm{PhD}$ degree.

This thesis is the result of a three-year research project during which curiosity, ambition, care and friendship took a central place. Throughout this project hard work was compensated for with a lot of serious fun and relaxation. I completed exciting journeys through the world of science as well as awesome trips around the globe.

This book is not the result of my individual effort alone. It is the result of teamwork. The team I am writing about is a special team and consists of friends, colleagues and family. These people mean a lot to me and deserve a special word of gratitude.

First of all, I would like to thank the many patients and volunteers who participated in the various studies that I completed for this thesis. I am grateful to your trust in me and to your willingness helping me in my attempt to improve patient care by generating scientific evidence.

During the past three years, I was supported by my promotores professor Kitslaar, professor van Engelshoven and my co-promotores doctor Tordoir and doctor Leiner. Professor Kitslaar, I highly appreciate your support and believe in me. It was you who excited my interest in hemodynamics and vascular surgery during my optional course in vascular surgery in my second year of medical school. Thank you for giving me the opportunity to explore my ideas under your supervision. Professor van Engelshoven, you are a generous person and a stimulating head of your department. You are an example to me. I would like to thank you for your involvement, hospitality and especially for encouraging me to start writing a research grant after finishing my master thesis. Doctor Tordoir, dear Jan, it was a privilege to work under your daily supervision. Your international name and fame in the field of vascular access surgery were of great help getting me in touch with people from all over the world. During the past three years you have given me all the space that I needed to expand my 
knowledge and to become involved in science. Your tolerance and support for some recalcitrant hypotheses meant a lot to me. Thank you. Doctor Leiner, dear Tim, after we first met during my interns I knew that our collaboration could lead to something precious. We did not only succeed in starting up trials and writing papers. You also became a friend. You are a born scientist and a source of inspiration. I learned a lot from you. I highly appreciate your devotion and valuable input in my work. I will always remember our scans during inhospitable times in the hospital while we had a lot of fun when other people tend to sleep.

I gratefully acknowledge all members of the reading committee for their time and effort: professor Hoeks, doctor Duijm, professor Leunissen and professor van de Vosse.

I would like to thank all the people who were involved in this multi-disciplinary project from the departments of surgery, radiology, nephrology, KEMTA and vascular lab (Maastricht University Hospital, Maastricht), department of Biophysics (Maastricht University, Maastricht), department of surgery, radiology, nephrology and vascular lab (Catharina Hospital Eindhoven), department of Cardiovascular Biomechanics (Biomedical Engineering, Eindhoven University of Technology, Eindhoven), Cardiovascular Mechanics and Biofluid Dynamics Research Unit (Ghent University, Gent, Belgium) and the Laboratory of Biomedical Technologies (Mario Negri Institute, Bergamo, Italy).

During my daily work, I very much enjoyed the company of room-mates, friends and colleagues, who were not all necessarily directly involved in this project. I think it is, however, impossible to thank you all personally in this preface. I do would like to thank you all because you often made my day by helping me get out of trouble or just being there, listening to my stupid ideas, my complaints or just making fun with me and making my stay in Maastricht pleasantly.

Paranimfen Robbert Nijenhuis and Luca Antiga, having you both next to me during my public defense means a lot to me. Dear Robbert, it was a pleasure having you as a close colleague and it is a privilege having you as a friend since our second year at medical school. Dear Luca, I will always remember our trip to Berlin. It was the perfect combination of geeky talk about vascular access, imaging, future plans and serious fun. You are an exceptional person and valuable 


\section{Preface}

friend and I am looking forward to our future collaborations and meetings accompanied by culinary delights.

In addition to all my friends (e.g. De Sneeuwkoningen) and colleagues, I would like to express my appreciation and gratitude to my family. Dear Aad en Joke (or vice versa naturally), I highly appreciate your unconditional interest and support in me during my whole life and particular during the years that I spent in Maastricht. You have been there for me when I needed you and you have let me go whenever necessary. Thank you for being great parents to me. Dear Karel, you are a special brother. Not because you are my only one but because of the person you are. I have learned a lot from you by observation and turned it into my profit. I admire your precision and animation in your work and I look forward to your public defense. Dear Beatrijs, my love, how on earth can I thank you? It makes me smile when I think of all the years that we spent together and I look forward to the years to come. Thank you for being who you are and for being yourself with me. 


Chapter 1

Introduction 


\section{Chapter 1}

\section{End-stage renal disease \& renal replacement therapy}

Progressive loss of renal function results in chronic renal failure. This can be defined as an abnormally low glomerular filtration rate $(<25 \mathrm{~mL} / \mathrm{min})$. The glomerular filtration rate can be determined indirectly from serum creatinine levels. Chronic renal failure is a severe illness and may lead to hypertension, uremia, hyperkalemia, anemia, edema, hyperphosphatemia, hypocalcemia, hyperparathyroidism and renal osteodystrophy which can be accompanied by symptoms of lethargy, malaise, cardiac arrhythmias, fatigue, and shortness of breath. If untreated, the disease will lead to death within days to weeks, depending on residual renal function. Chronic renal failure that leads to severe illness, which requires renal transplantation or some form of renal replacement therapy (peritoneal dialysis or hemodialysis), is called end-stage renal disease (ESRD).

\section{Facts \& figures}

In the year 2004, the number of ESRD patients was 1,783,000 world wide, 437,000 and 11,002 in Europe and The Netherlands, respectively. Because of a shortage of donor kidneys, the majority of ESRD-patients are treated by renal replacement therapy, e.g. hemodialysis (69\%) and peritoneal dialysis (8\%). The number of ESRD-patients increases continuously and in particular the number of patients older 65 years. The fraction of patients receiving a renal transplant decreases with age. Conversely, the percentage of patients being treated by hemodialysis increases with age. Over the next few years the number of ESRDpatients is expected to increase by aproximately $5 \%$ per year reaching over $3,000,000$ world-wide by 2010 . Because of the chronic nature of the disease this increase will be accompanied by a large increase in healthcare related costs (1$3)$.

\section{Hemodialysis treatment principles}

Hemodialysis is a method to remove waste products as potassium, urea and free water from blood. To enable hemodialysis, a system of tubes connects the patient to a semi-permeable membrane with dialysis fluid running on the other side. The blood passes the semi-permeable membrane and the cleansed blood is then returned via the circuit back into the bloodstream of the patient as shown in 
figure 1. Ultrafiltration is accomplished by increasing the hydrostatic pressure of the blood in the dialysis circuit resulting in a pressure gradient that drives the water ecxess across the memebrane. The dialysis process is very efficient, allowing treatment to be carried out intermittently, usually three times a week.

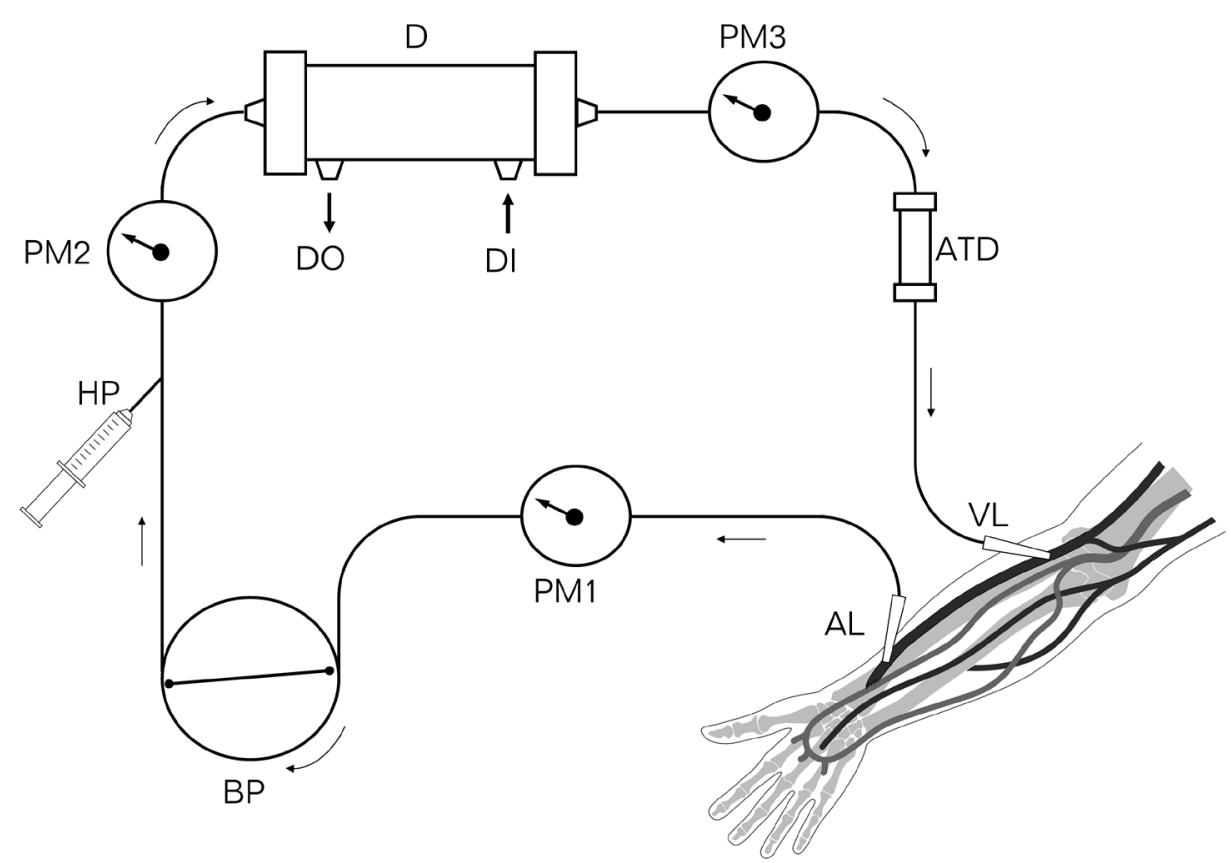

Figure 1: Schematic representation of the hemodialysis circuit. The patient is connected to the dialyzer by two cannulas which are connected to an arterial line and a venous line. Blood is removed from the patient for cleansing in the dialyzer and thereafter returned to the patient. Legend: $A L=$ arterial line, $\mathrm{VL}=$ venous line, $\mathrm{PM} 1=$ arterial pressure monitor, $\mathrm{PM} 2=$ dialyzer pressure monitor, $\mathrm{PM} 3=$ venous pressure monitor, $\mathrm{BP}=$ blood pump, $\mathrm{HP}=$ heparine pump to prevent blood clotting, $\mathrm{D}$ $=$ dialyzer, $\mathrm{DI}=$ dialysate inflow, $\mathrm{DO}=$ dialysate outflow, $\mathrm{ATD}=$ air trap and detector. 


\section{Chapter 1}

\section{Vascular access for hemodialysis}

A functioning vascular access is a prerequisite for successful hemodialysis treatment. There are three modes to gain vascular access: an autogenous arteriovenous fistula (AVF), an arteriovenous graft (AVG), and a central venous catheter (CVC). The type of access chosen for an individual patient is influenced by factors such as the expected time course of the patient's renal failure and the condition of the vasculature (4-7).

\section{Autogenous arteriovenous fistula}

The AVF is the preferred access method and is made by connecting an artery with a vein by a surgically created anastomosis. This anastomosis bypasses the capillary bed and directly connects the high pressure arterial system to the low pressure and low resistant venous system, which initiates the maturation process. A very high blood flow high will develop within minutes to hours after creation of the arteriovenous anastomosis. The draining vein will dilate due to the increase in flow and subsequent increase in venous intravascular pressure. Both flow increase and venous dilatation are crucial for successful AVF maturation to obtain a functioning hemodialysis vascular access. Venous dilatation facilitates repetitive cannulation with large caliber needles and the high flow rates enable adequate extra-corporal perfusion of the dialyser for effective hemodialysis treatment. AVFs are usually created in the non-dominant arm, and may be situated at the level of the wrist (the 'snuffbox' fistula'), the forearm, usually a radiocephalic AVF, in which the radial artery is anastomosed to the cephalic vein as shown in figure 2. Alternatively an AVF can be created in the elbow, usually a brachiocephalic fistula, where the brachial artery is anastomosed to the cephalic vein. The maturation process, venous dilatation and flow rate increase, generally takes about a few weeks. During dialysis, two needles are inserted into the venous side of the fistula, one to draw blood from the patient and one to return the blood to the patient. However, single needle dialysis is also possible, being less effective though. The advantages of AVFs over other types of vascular accesse are the lower infection rate and a lower incidence of thrombosis. The main limitation of AVFs is the chance of inadequate blood flow increase or inadequate venous dilatation after creation, resulting in an AVF which cannot be used for hemodialysis treatment (4-8). 


\section{Arteriovenous graft}

The AVG is much like the AVF in most respects, except that a graft, often made of a synthetic material, is used to join the artery and vein. An AVG should only be created when the patient's native vasculature does not permit AVF creation. AVGs mature faster than AVF and may be used within hours to days after creation. However, AVGs are at high risk for developing stenosis at the sites of arterial and venous anastomosis and within the graft. Stenosis development causes flow decline and subsequently thrombosis of the AVG. Due to the use of foreign material, AVGs are at greater risk for becoming infected when compared to AVFs. In figure 2 a schematic representation of a forearm loop AVG is shown (4-7).

\section{Central venous catheter}

The central venous catheter generally consists of a double lumen plastic catheter which is inserted into a large vein (usually the vena cava, via the internal jugular vein, the subclavian vein or the femoral vein) to allow large volumes of blood to be rapidly withdrawn from the arterial lumen, to go into the dialysis circuit, and to be returned via the venous lumen. Blood flow rates are usually lower compared to a well functioning AVF or AVG. Aside from infection, central vein stenosis is a serious problem associated with CVCs. This can lead to severe venous congestion in the area drained by the affected vein, in particular after creation of an ipsilateral AVF or AVG. Therefore CVCs are mainly used for immediate access in patients in need of urgent dialysis or for patients who are deemed likely to recover from acute renal failure in addition to patients with ESRD waiting for a permanent vascular access (4-7). 


\section{Chapter 1}
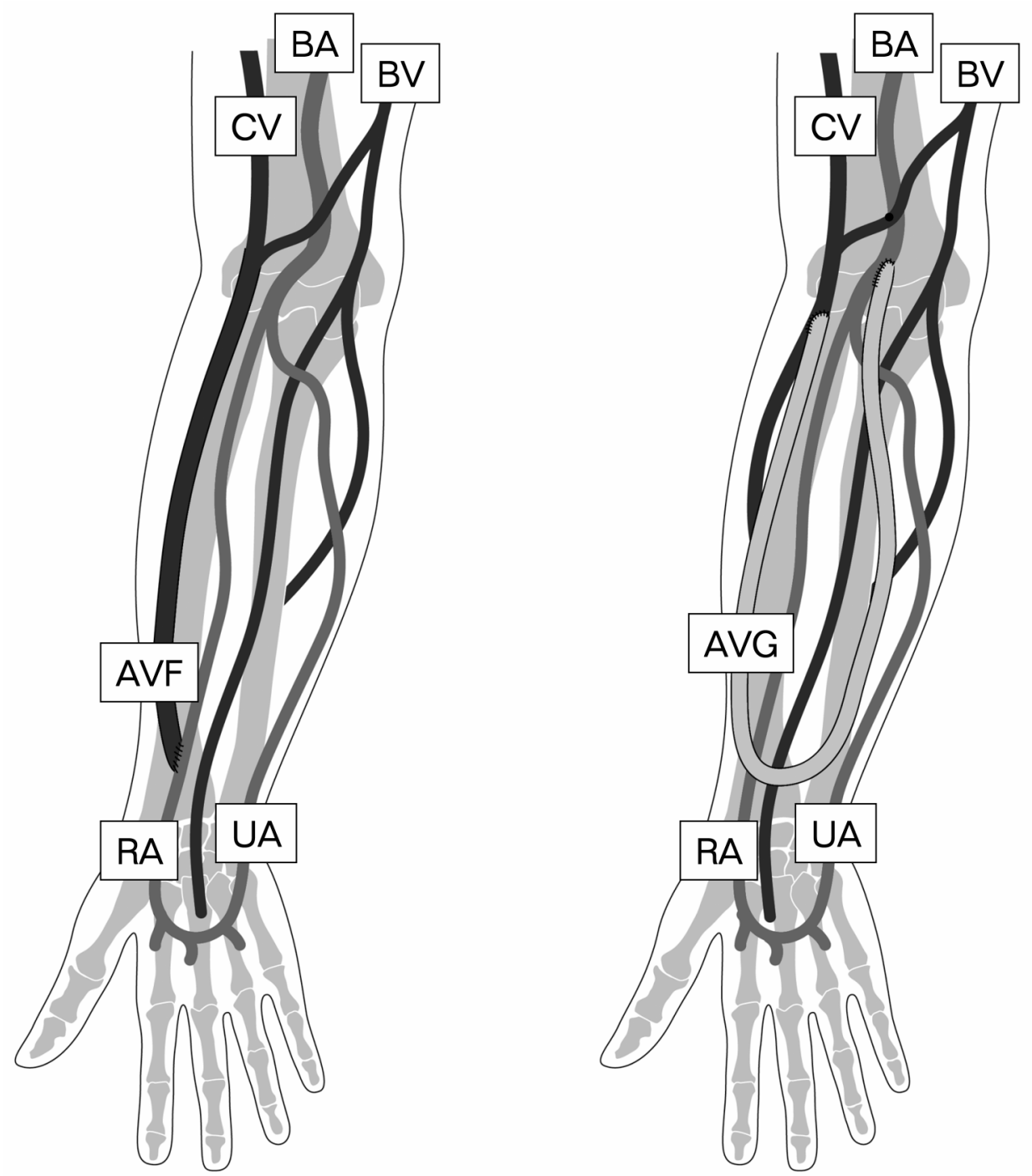

Figure 2: Schematic drawing of an autogenous radiocephalic AVF (left) and a prosthetic forearm loop AVG (right). Legend: $B A=$ brachial artery, $R A=$ radial artery, $U A=$ ulnar artery, $C V=$ cephalic vein, $\mathrm{BV}=$ basilic vein, $\mathrm{AVF}=$ arteriovenous fistula, anastomosis of radial artery (side) to cephalic vein (end) at the wrist, $A V G=$ arteriovenous loop-graft anastomosed to brachial artery and cephalic vein at the antecubital crease). 


\section{Tailoring the vascular access type to the patient}

When hemodialysis treatment is anticipated, it has to be decided which type of vascular access is best for the patient. Several health aspects such as the condition of the cardiovascular system, co-morbidities and life expectancy are of importance (6). The AVF is the first choice vascular access because of its superior long-term performance when compared to CVCs and AVGs. AVGs are associated with approximately five times more interventions to keep the access functioning, leading to higher vascular access related morbidity, mortality and healthcare costs, when compared to AVFs (7, 9-27). Guidelines all over the world recommend creation of AVFs instead of inserting CVCs or AVGs $(4,6,7)$. However, the clinical success of AVFs is jeopardized by non-maturation after initial creation (28-35). Non-maturation can be defined as an AVF being inadequate for hemodialysis access use due to insufficient flow increase or venous distension postoperatively. Non-maturation has been reported to be associated with patient characteristics (i.e. female gender, older age, diabetes, obesity, cardiovascular morbidity, previous vascular access procedures, previous limb or thorax surgery and radiation therapy) and preoperative arterial and venous characteristics such as static parameters (i.e. diameter) and dynamic (i.e. flow, flow velocity, compliance, resistive index and response to hyperemia or congestion) (6, 32, 36-39). Furthermore the presence of venous stenosis and venous side branches may also jeopardize AVF maturation due to poor venous outflow or disadvantageous flow distributions, respectively $(6,7,35,40,41)$. Despite the application of preoperative assessment, however, non-maturation rates remain high up to $50 \%$ (28-35).

\section{The current thesis}

The following chapters address the clinical value of diagnostic modalities used for preoperative assessment of upper extremity vessels with respect to access creation and maturation. Furthermore, the clinical value of contrast-enhanced magnetic resonance angiography as part of a vascular access surveillance program for detection of flow-limiting stenoses is assessed. 


\section{Chapter 1}

\section{Aims of this thesis are}

1. To review the current diagnostic work-up of ESRD-patients prior to vascular access creation and the parameters used to predict and prevent nonmaturation of newly created AVFs.

2. To determine duplex ultrasound forearm superficial venous diameter measurement reproducibility and the effect of venous congestion on diameter and diameter measurement reproducibility.

3. To assess the feasibility of a contrast-enhanced magnetic resonance angiography protocol for selective assessment of the upper extremity arteries and veins in a single exam.

4. To prospectively determine the value of contrast-enhanced magnetic resonance angiography for prediction of vascular access early failure and non-maturation.

5. To prospectively determine the clinical significance of accessory veins in the light of RC-AVF non-maturation.

6. To assess the diagnostic performance of multiphase contrast-enhanced magnetic resonance angiography for detection of flow limiting stenosis in surgically created AVFs and AVGs. 


\section{Overview of this thesis}

Chapter 2 reviews the diagnostic work-up prior to hemodialysis vascular access creation regarding the available diagnostic modalities for upper extremity vessel assessment. The relation between venous congestion pressures and forearm superficial venous cross sectional area shape and size is addressed in chapter 3 . Thereafter the effect of venous congestion methods on forearm superficial venous diameter measurement reproducibility is assessed in chapter 4. Chapter 5 reports the feasibility of a contrast-enhanced magnetic resonance angiography protocol for the assessment of upper extremity arteries and veins prior to vascular access. A prospective analysis of preoperatively determined duplex ultrasonography and contrast-enhanced magnetic resonance angiography parameters in relation to clinical outcome is reported in chapter 6 . The clinical value of accessory veins for prediction of RC-AVF non-maturation is determined in chapter 7. The diagnostic performance of multiphase contrast-enhanced magnetic resonance angiography for the detection of flow limiting stenoses, compared to digital subtraction angiography as standard of reference, is reported in chapter 8. Chapter 9 includes a general discussion, future perspective and conclusion of this thesis. Chapter 10 summarizes this thesis in Dutch.

The research for this thesis was performed at the departments of surgery, radiology and nephrology at Maastricht University Hospital and Cardiovascular Research Institute Maastricht (CARIM), in collaboration with the departments of surgery, radiology and nephrology at Catharina Ziekenhuis Eindhoven, The Netherlands. The project was funded by grant P189 of the Maastricht University Hospital Profileringsfonds. 


\section{Chapter 1}

\section{References}

1. Moeller S, Gioberge S, Brown G. ESRD patients in 2001: global overview of patients, treatment modalities and development trends. Nephrol Dial Transplant 2002; 17:2071-2076.

2. Grassmann A, Gioberge S, Moeller S, Brown G. ESRD patients in 2004: global overview of patient numbers, treatment modalities and associated trends. Nephrol Dial Transplant 2005; 20:2587-2593.

3. Meichelboeck W. ESRD 2005 - A Worldwide Overview - Facts, Figures and Trends. In: Mickley $V$, ed. 4th International Congress of the Vascular Access Society (VAS). Berlin, Germany: Blood Purification, Karger, 2005; 227-261.

4. Clinical practice guidelines for vascular access. Am J Kidney Dis 2006; 48 Suppl 1:S176-247.

5. Wilson SE. Vascular Access: Principles and Practice. St Louis, Missouri: Mosby - Year Book, Inc., 1996.

6. Tordoir JH, Mickley V. European guidelines for vascular access: clinical algorithms on vascular access for haemodialysis. Edtna Erca J 2003; 29:131-136.

7. III. NKF-K/DOQI Clinical Practice Guidelines for Vascular Access: update 2000. Am J Kidney Dis $2001 ; 37: S 137-181$.

8. Strandness DE, Sumner DS. Hemodynamics for Surgeons. New York: Grune \& Stratton, 1975.

9. Anel RL, Yevzlin AS, Ivanovich P. Vascular access and patient outcomes in hemodialysis: questions answered in recent literature. Artif Organs 2003; 27:237-241.

10. Besarab A, Brouwer D. Improving arteriovenous fistula construction: Fistula firs initiative. Hemodialysis International 2004; 8:199-206.

11. Brescia MJ, Cimino JE, Appel K, Hurwich BJ. Chronic hemodialysis using venipuncture and a surgically created arteriovenous fistula. N Engl J Med 1966; 275:1089-1092.

12. Dhingra RK, Young EW, Hulbert-Shearon TE, Leavey SF, Port FK. Type of vascular access and mortality in U.S. hemodialysis patients. Kidney Int 2001; 60:1443-1451.

13. Harland RC. Placement of permanent vascular access devices: surgical considerations. Adv Ren Replace Ther 1994; 1:99-106.

14. Konner K. The initial creation of native arteriovenous fistulas: surgical aspects and their impact on the practice of nephrology. Semin Dial 2003; 16:291-298.

15. Konner K, Nonnast-Daniel B, Ritz E. The arteriovenous fistula. J Am Soc Nephrol 2003; 14:1669-1680.

16. Lee $\mathrm{H}$, Manns $\mathrm{B}$, Taub $\mathrm{K}$, et al. Cost analysis of ongoing care of patients with end-stage renal disease: the impact of dialysis modality and dialysis access. Am J Kidney Dis 2002; 40:611-622.

17. Nassar GM, Ayus JC. Infectious complications of the hemodialysis access. Kidney Int 2001; 60:1-13.

18. Pisoni RL, Young EW, Dykstra DM, et al. Vascular access use in Europe and the United States: results from the DOPPS. Kidney Int 2002; 61:305-316. 
19. Polkinghorne KR, McDonald SP, Atkins RC, Kerr PG. Vascular access and all-cause mortality: a propensity score analysis. J Am Soc Nephrol 2004; 15:477-486.

20. Rayner HC, Pisoni RL, Bommer J, et al. Mortality and hospitalization in haemodialysis patients in five European countries: results from the Dialysis Outcomes and Practice Patterns Study (DOPPS). Nephrol Dial Transplant 2004; 19:108-120.

21. Sands J, Perry M. Where are all the AV fistulas? Semin Dial 2002; 15:146-148.

22. Taylor G, Gravel D, Johnston L, Embil J, Holton D, Paton S. Prospective surveillance for primary bloodstream infections occurring in Canadian hemodialysis units. Infect Control Hosp Epidemiol 2002; 23:716-720.

23. Woods JD, Port FK. The impact of vascular access for haemodialysis on patient morbidity and mortality. Nephrol Dial Transplant 1997; 12:657-659.

24. Xue JL, Dahl D, Ebben JP, Collins AJ. The association of initial hemodialysis access type with mortality outcomes in elderly Medicare ESRD patients. Am J Kidney Dis 2003; 42:1013-1019.

25. Staramos DN, Lazarides MK, Tzilalis VD, Ekonomou CS, Simopoulos CE, Dayantas JN. Patency of autologous and prosthetic arteriovenous fistulas in elderly patients. Eur J Surg 2000; 166:777781.

26. Kherlakian GM, Roedersheimer LR, Arbaugh JJ, Newmark KJ, King LR. Comparison of autogenous fistula versus expanded polytetrafluoroethylene graft fistula for angioaccess in hemodialysis. Am J Surg 1986; 152:238-243.

27. Leapman SB, Boyle M, Pescovitz MD, Milgrom ML, Jindal RM, Filo RS. The arteriovenous fistula for hemodialysis access: gold standard or archaic relic? Am Surg 1996; 62:652-656; discussion 656-657.

28. Huber TS, Ozaki CK, Flynn TC, et al. Prospective validation of an algorithm to maximize native arteriovenous fistulae for chronic hemodialysis access. J Vasc Surg 2002; 36:452-459.

29. Allon M, Robbin ML. Increasing arteriovenous fistulas in hemodialysis patients: problems and solutions. Kidney Int 2002; 62:1109-1124.

30. Patel ST, Hughes J, Mills JL, Sr. Failure of arteriovenous fistula maturation: an unintended consequence of exceeding dialysis outcome quality Initiative guidelines for hemodialysis access. J Vasc Surg 2003; 38:439-445; discussion 445.

31. Miller PE, Tolwani A, Luscy CP, et al. Predictors of adequacy of arteriovenous fistulas in hemodialysis patients. Kidney Int 1999; 56:275-280.

32. Mihmanli I, Besirli K, Kurugoglu S, et al. Cephalic vein and hemodialysis fistula: surgeon's observation versus color Doppler ultrasonographic findings. J Ultrasound Med 2001; 20:217222.

33. Robbin ML, Gallichio MH, Deierhoi MH, Young CJ, Weber TM, Allon M. US vascular mapping before hemodialysis access placement. Radiology 2000; 217:83-88.

34. Tordoir JH, Rooyens P, Dammers R, van der Sande FM, de Haan M, Yo TI. Prospective evaluation of failure modes in autogenous radiocephalic wrist access for haemodialysis. Nephrol Dial Transplant 2003; 18:378-383. 


\section{Chapter 1}

35. Ascher E, Gade $P$, Hingorani $A$, et al. Changes in the practice of angioaccess surgery: impact of dialysis outcome and quality initiative recommendations. J Vasc Surg 2000; 31:84-92.

36. Konner K, Hulbert-Shearon TE, Roys EC, Port FK. Tailoring the initial vascular access for dialysis patients. Kidney Int 2002; 62:329-338.

37. Malovrh M. Non-invasive evaluation of vessels by duplex sonography prior to construction of arteriovenous fistulas for haemodialysis. Nephrol Dial Transplant 1998; 13:125-129.

38. Malovrh M. Native arteriovenous fistula: preoperative evaluation. Am J Kidney Dis 2002; 39:1218-1225.

39. Zeebregts C, van den Dungen J, Bolt A, Franssen C, Verhoeven E, van Schilfgaarde R. Factors predictive of failure of Brescia-Cimino arteriovenous fistulas. Eur J Surg 2002; 168:29-36.

40. Turmel-Rodrigues L, Mouton A, Birmele B, et al. Salvage of immature forearm fistulas for haemodialysis by interventional radiology. Nephrol Dial Transplant 2001; 16:2365-2371.

41. Beathard GA, Arnold P, Jackson J, Litchfield T. Aggressive treatment of early fistula failure. Kidney Int 2003; 64:1487-1494. 




\section{Chapter 2}

Current techniques for assessment of upper extremity vasculature prior to hemodialysis vascular access creation

R.N. Planken, J.H.M. Tordoir, L.E.M. Duijm, M.W. de Haan, T. Leiner

European Radiology: in press 


\begin{abstract}
Vascular access problems lead to increased patient morbidity and mortality and place a large burden on care facilities, manpower and costs. Autogenous arteriovenous fistulas (AVFs) are preferred over arterio-venous grafts (AVGs) because of a lower incidence of vascular access related complications. An aggressive increase in the utilization of AVFs, however, results in an increased incidence of AVF early failure and non-maturation. Increasing evidence suggests that routine preoperative assessment results in an increased utilization of functioning AVF by better selection of adequate vessels. To date, the reproducibility and standardization of assessment protocols are lacking and assessment of a single morphological parameter has not enabled adequate prediction of postoperative AVF function for individual patients. In this paper we provide an overview of available diagnostic modalities and parameters that potentially enable better selection of adequate vessels for successful AVF creation.
\end{abstract}




\section{Introduction}

End-stage renal disease (ESRD), defined as complete or near complete renal failure, is an increasingly important medical problem, ultimately requiring hemodialysis in the vast majority of patients. The incidence and prevalence of end-stage renal disease (ESRD) as well as the number of patients requiring hemodialysis have sharply risen over the past few years. In 2004, an estimated 1.22 million patients were on hemodialysis world-wide, representing a $20 \%$ increase in three years since $2001(1,2)$.

A well functioning vascular access is the cornerstone of hemodialysis treatment in ESRD patients and can be achieved by either insertion of a central venous catheter or by surgical creation of an arteriovenous fistula or graft. The access of first choice is the autogenous arteriovenous fistula (AVF) because it has better long-term performance and patency rate when compared to arteriovenous graft (AVG) or central venous catheter (CVC). Furthermore, AVFs have lower vascular access related morbidity, mortality and healthcare costs compared to AVGs and CVCs (3-19). AVGs require about five times more therapeutic interventions compared to AVFs, to keep the access functioning (20-22).

In order to reduce long-term vascular access related complications, the Dialysis Outcome Quality Initiative (K-DOQI) and Good Nephrological Practice guidelines advocate an all-AVF policy, i.e. at least $70 \%$ of all newly created accesses should consist of autogenous AVFs $(3,4,23)$. However the major drawback of AVFs creation is the relatively high frequency of early thrombosis - up to $10-20 \%$ of all newly created vascular accesses thrombose within the first week after creation and non-maturation $(24,25)$. Non-maturation is defined as an AVF being inadequate for hemodialysis due to insufficient flow or insufficient venous distension within months after creation. Causes of non-maturation are thought to be the use of tiny vessels in addition to arterial inflow or venous outflow stenoses or occlusions (25-29). Furthermore, the presence of large caliber side branches may also jeopardize AVF maturation due to a disadvantageous flow distribution $(4,30,31)$. Different studies have reported AVF non-maturation rates within the first months after creation from $5 \%$ up to $54 \%(28,32-37)$. 
In order to increase the number of mature and functional AVFs, adequate history taking, physical examination and preoperative assessment of upper extremity vessels are important to potentially prevent AVF early failure and non-maturation $(3,4,23,36)$. Increasingly, arterial and venous diameters as well as the presence and location of preexisting atherosclerotic occlusive disease and venous stenoses, occlusions and side-branches are used to guide the choice of fistula type and location. Consequently, interest has risen in preoperative imaging of upper extremity vessels. The goal of preoperative imaging is assessment of vessel caliber and identification of sites where arteries and veins are of suboptimal quality for access purposes.

The current review will provide the radiologist with an overview of the clinical role and relative merits and shortcomings of physical examination, duplex ultrasonography (DUS), digital subtraction angiography (DSA) and contrastenhanced magnetic resonance angiography (CE-MRA) in the preoperative workup of patients awaiting surgical creation of a vascular access for hemodialysis.

\section{History taking and physical examination}

Underlying any pre-surgical workup is a thorough history and physical examination. Females, elderly patients and patients suffering from diabetes mellitus, obesity, cardiovascular morbidity, and patients with a history of previous vascular access procedures (CVC, AVF and AVG) as well as previous limb and thoracic surgery or radiation therapy, are at increased risk of AVF non-maturation (23). Physical examination is an important and sometimes valuable tool in the work-up of patients awaiting vascular access surgery. Skin lesions, local infections, generalized dermatological problems and scars may indicate poor chance of successful AVF creation at standard locations and should therefore be addressed.

\section{Arterial assessment}

Signs of peripheral ischemia such as arm claudication, secondary Raynaud's phenomenon and necrosis are important indicators of arterial occlusive disease with increased risk of AVF malfunction and ischemic complications postoperatively (23). Vigorousness of arterial pulsations in the brachial (just proximal to the elbow), radial and ulnar arteries (just proximal to the wrist) are reasonable 
proxy measures for the quality of the arterial tree, although no information about palmar arch patency can be obtained by palpation of pulsations only. In addition to palpation of arterial pulsations, many clinicians still use the Allen test, introduced in 1929 as a simple and reliable test to assess the patency of the palmar arches of the hand (38). The Allen test relies on prompt return of color to the blanched hand upon release of a tightly clenched fist and concomitant release of compression of radial and ulnar arteries to indicate patent palmar arches. However, McGregor found that even in the presence of ulnar artery occlusion, perfusion of the entire hand was immediately redirected via the radial artery in cases in which the Allen test suggested a non-patent radial artery (39). The Allen test, furthermore, shows poor clinical performance for detection of palmar arch occlusion when compared to duplex ultrasonography (sensitivity $28.6 \%$ [95\% Cl: 3.7-71.0\%]; specificity 96.6\% [95\% Cl: 88.3-99.6\%]; PPV 50\% [95\% Cl: 6.893.2\%] and NPV 91.9\% [Cl: 82.2\%-97.3\%]) (40). Therefore, the use of the Allen test in the workup prior to vascular access creation remains controversial and should be considered of little, if any, clinical value.

Another frequently used method to assess upper extremity arteries are bilateral blood pressure measurements. A difference of $>20 \mathrm{mmHg}$ or an arm-arm index $<0.9$ are indicative for the presence of proximal arterial pathology (23).

\section{Venous assessment}

The superficial venous system should be assessed before and after application of a proximal tourniquet to determine venous compliance. Venous continuity, caliber, compliance, compressibility and the presence of accessory veins determine the suitability of each vein for AVF use. Small caliber veins, venous obstructions, low compliance and the presence of larger accessory veins are associated with higher non-maturation rates $(3,4,23)$. Furthermore, arm swelling or the presence of collateral veins is indicative for the presence of a central venous obstruction. However, absence of arm swelling or the absence of visible collateral veins does not imply the absence of a central venous obstruction. In case of abnormal findings during physical examination further diagnostic procedures are indicated (23).

Although physical examination can be a clinically valuable preoperative diagnostic tool, it should be recognized that it is challenging and of limited value in obese 
patients $(28,41)$. Malovrh et al. found that physical examination failed to identify suitable vessels for AVF creation in over half of all patients undergoing dialysis access surgery ( $n=62 / 116 ; 54 \%$ ) (25). The results of physical examination should therefore be interpreted with caution because of low sensitivity, specificity, positive predictive value and negative predictive values of $40 \%, 81 \%$, $50 \%$ and $74 \%$ for prediction of AVF function, respectively (42).

\section{Duplex ultrasonography}

DUS enables assessment of vessel parameters such as patency, diameter, flow and flow-velocities. The application of DUS enables better depiction of adequate vessels for AVF creation that may not be detected by physical examination, especially in obese patients $(25,28,41,43)$. Multiple studies have found that the application of DUS resulted in changes in surgical procedure in $31 \%$ of cases, changes in site of exploration (9.6\%), a decrease in unsuccessful explorations (11\% to $0 \%$ ), and an increase in the relative number of AVFs created (as opposed to other types of access; $64 \%$ vs $34 \%$ ), and a decrease in non-maturation rates (38\% to $8.3 \%$ and $66 \%$ to $46 \%$ ), when compared to the use of physical examination alone $(28,33,44-46)$. However, reported cut-off values of various DUS-derived parameters (i.e. diameter, flow, flow-velocities, compliance and resistive index) are inconsistent. An aggressive approach to conform to the allAVF policy may therefore still result in increased early failure and non-maturation rates if based on a single DUS-derived parameter $(47,48)$. It remains to be established wether a combination of parameters might enable better prediction of vascular access function and minimize early failure and non-maturation rates.

\section{Arterial assessment}

Preoperative DUS examination should include assessment of the arteries from the infraclavicular subclavian artery down to the radial and ulnar arteries at the wrist (23). The exact course and continuity as well as the presence of stenoses should be addressed because patients with arterial stenosis are thought to be at increased risk for developing hand and finger ischemia after AVF creation due to steal phenomena (23). For detection of stenoses ( $>50 \%$ luminal reduction) in the upper extremity arterial system, DUS has a sensitivity and specificity of $90.9 \%$ and $100 \%$ for the subclavian artery, $93.3 \%$ and $100 \%$ for upper arm arteries, 
$88.6 \%$ and $98.7 \%$ for forearm arteries and for the arteries of the hand $70 \%$ and $100 \%$, respectively $(49,50)$.

Another important morphological parameter, apart from the presence of arterial stenosis is arterial diameter. Arterial diameters smaller than 1.5 to $3.0 \mathrm{~mm}$ have been associated with increased non-maturation rates of AVFs $(27,42,45,46,51$ 53). Malovrh et al. found that an arterial cut-off diameter $>1.6 \mathrm{~mm}$ may result in a sensitivity of $93 \%$ and specificity of $68 \%$ with positive - and negative predictive values of $86 \%$ and $74 \%$ respectively, for the prediction of successful AVF maturation (25). In other studies different cut-off values have been suggested, $<1.5 \mathrm{~mm}$ (27), >1.6 mm (42), >2.0 mm (46), $2.75 \mathrm{~mm}$ (52) and $3.0 \mathrm{~mm}$ (53). Preoperative DUS arterial diameters measurements correlate well with operative diameter measurements although they reflect a slight overestimation (27). DUS has been reported to be highly accurate for diameter measurement of vessels $>1.5 \mathrm{~mm}$, with quantitative measurements possible to a resolution of $0.5 \mathrm{~mm}$ (54).

In addition to static diameter measurements, several other dynamic DUS-derived preoperative parameters such as radial artery flow and peak systolic velocities before or during reactive hyperemia have been reported to be valuable predictors of AVF maturation. In figure 1, radial artery flow velocity changes due to fist clenching and reactive hyperemia are shown. Absence of change or a diminished change in radial artery flow-velocity is associated with a higher risk of vascular access early failure and non-maturation. Lockhart et al., however, found that arterial diameters, resistance indices and peak systolic velocities had only little, if any, predictive value for AVF outcome (48).

An important caveat of the reported clinical value of DUS derived parameters is the difference in results within and between research groups with respect to cutoff values and to the predictive value of the aforementioned parameters $(25,52$, $55,56)$. 

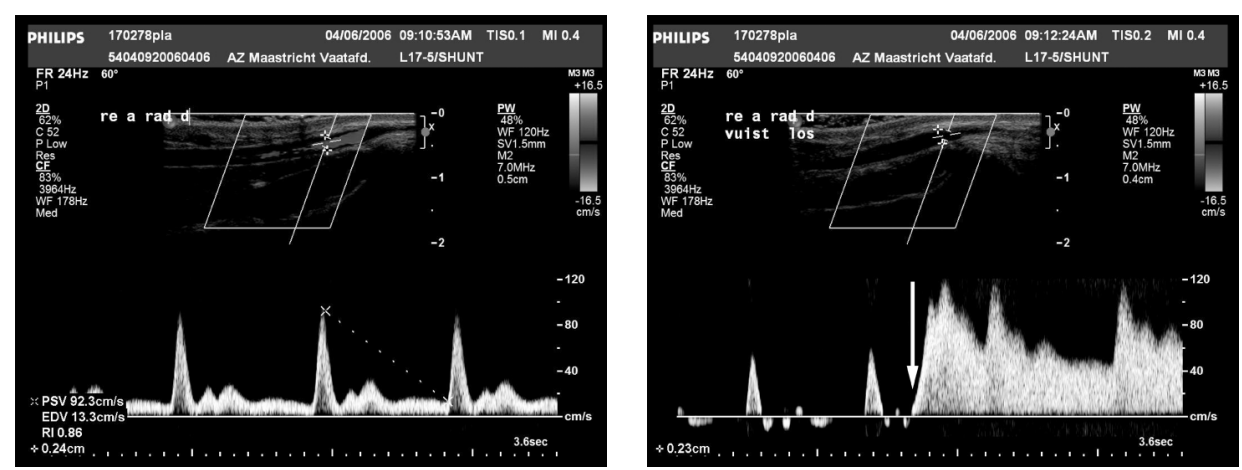

Figure 1: Duplex ultrasound assessment of radial artery flows-velocitiesat rest in a healthy volunteer (left). Ratial artery flow velocities in the same person during fist clenching and during hyperemia after the fist clench has been released (right). The arrow indicates the moment of fist clench release.

\section{Venous assessment}

The superficial venous system of the upper extremity is easily assessable by DUS and results in detection of more veins compared to physical examination alone (28, 41, 43). Furthermore, it also allows for assessment of local hemodynamics, such as subclavian vein flow. A typical example of Doppler signal changes of the subclavian vein during deep inspiration in a healthy volunteer is shown in figure 2 . The absence of changes in venous Doppler signal due to deep inspiration or loss of venous compressibility is another important finding indicative for local venous stenosis or occlusion.

Preoperative detection of stenoses and obstructions is important to avoid unsuccessful surgical explorations for vascular access creation (23). Nack et al. reported a DUS sensitivity, specificity, positive -, and negative predictive value of $81 \%, 90 \%, 90 \%$ and $78 \%$ respectively, for detection of venous stenosis, thrombi and occlusions when compared to DSA (57).

The clinical value of upper extremity DUS for detection of venous abnormalities is lower for proximal compared to distal veins (sensitivity and specificity: proximal veins $63 \%$ and $97 \%$, distal veins $94 \%$ and $97 \%$ ) (50). These results are in agreement with the results of Nack et al. who reported different DUS sensitivities for detection of abnormalities in the subclavian vein (79\%), innominate vein $(75 \%)$, and superior vena cava (33\%), when compared to DSA (57). 


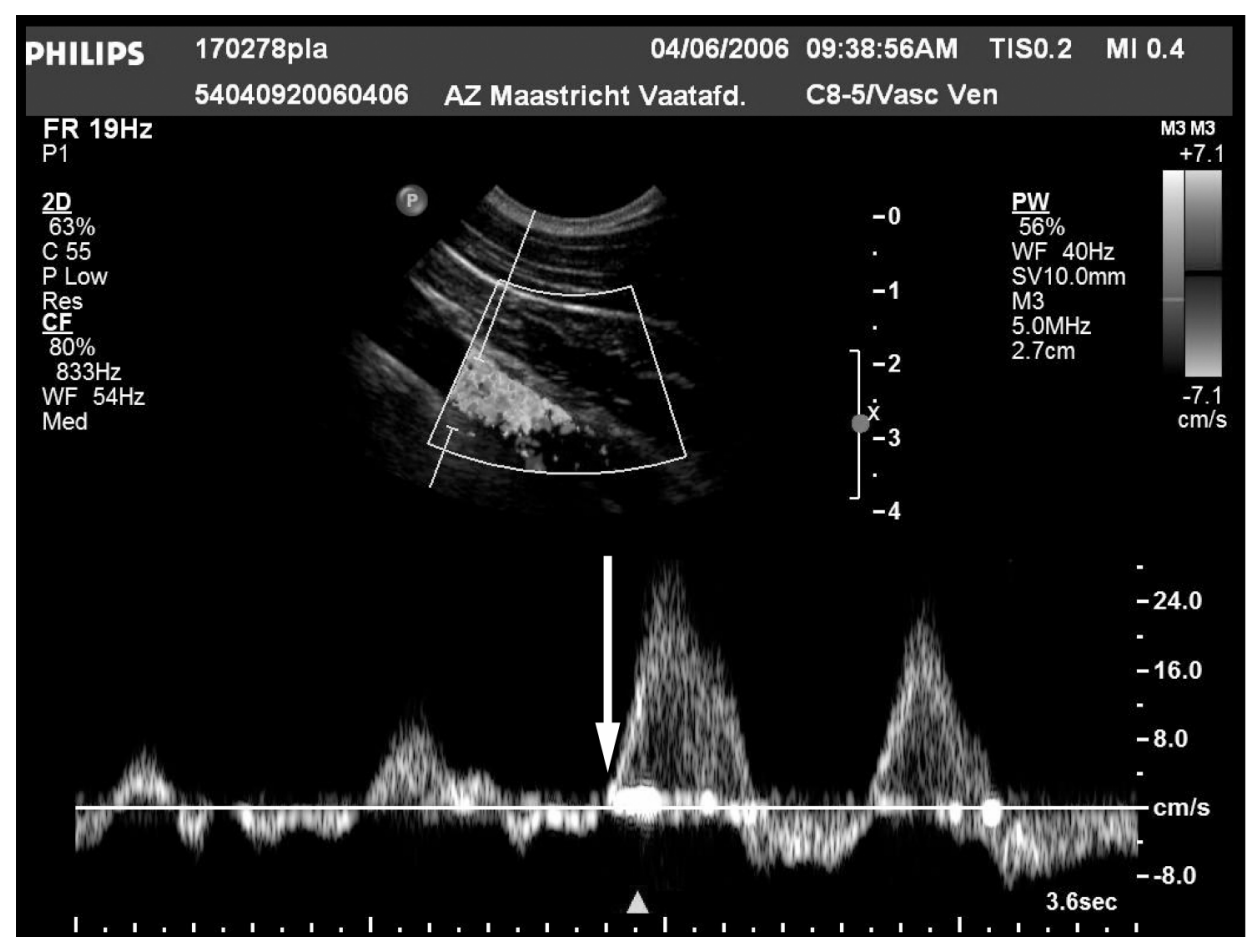

Figure 2: The effect of deep inspiration on subclavian vein flow-velocities assessed with duplex ultrasound. The arrow indicates the start of deep inspiration. Absence or diminished subclavian vein flow-velocities or no change in flow-velocities due to deep inspiration is associated with a higher risk of vascular access early failure and non-maturation.

As is the case for arteries, DUS derived venous diameters have also been reported as an important parameter to predict vascular access outcome. For assessment of venous diameter, a proximally applied cuff should be used to induce venous dilatation for better appreciation of 'maximum' or 'true' venous diameter $(3,4,23)$. Venous diameters smaller than 1.6 to $2.6 \mathrm{~mm}$ have been associated with AVF failure $(25,36,42,45,46,51,58)$. This range may be partially explained by differences in vein mapping protocols because only few authors reported the measurement conditions and the methods that they used to achieve venous dilatation. Furthermore, DUS venous diameter measurements are observer-dependent with an inter-observer variation of $0.5 \mathrm{~mm}$ (59). Recently, Planken et al. have demonstrated that superficial forearm vein diameter measurements vary over time with a coefficient of variation of $27 \%$ (60). In addition, forearm superficial venous diameter measurement reproducibility 


\section{Chapter 2}

depends on the applied venous congestion pressure. Best reproducibility is achieved at venous congestion pressures $>40 \mathrm{mmHg}$ (61). Other research groups, however, suggested that the contiguous length of non-diseased vein $>10 \mathrm{~cm}$ and not diameter of the superficial vein was predictive for the AVF function $(52,62)$.

Apart from venous diameter, some authors have found an association between the presence and size of venous side branches and AVF non-maturation. Wong et al. suggested that a side branch $<5 \mathrm{~cm}$ away from the planned anastomosis may impair AVF function, whereas Beathard et al. have stressed the importance of the size of the venous side branches. In these studies non-maturation was more likely in the event of a large venous side branch $(31,42)$. Turmel-Rodrigues et al., in contrast, state that venous side branches are of no importance and only come into play in the presence of a venous outflow stenosis (30).

Dynamic parameters to characterize upper extremity veins include flow and velocity measurements as well as assessment of flow-velocity changes due to respiratory maneuvers (23). The capacity of superficial veins to dilate due to venous congestion (also known as compliance) has been reported to be higher within a group of patients in whom AVF creation was successful compared to patients with AVF that failed to mature (diameter increase $48 \%$ versus $12 \%$ at a congestion pressure of $50 \mathrm{mmHg}$ ) (25). Forearm superficial venous compliance measurements are, however, poorly reproducible due to the poor reproducibility of venous diameters at low venous congestion pressures (61). The clinical value of forearm superficial venous compliance measurements is therefore considered of little if any use.

The preoperative DUS exam of both arteries and veins has a sensitivity, specificity, positive predictive value and negative predictive value of $20-44 \%$, 94$100 \%, 60-100 \%$ and $73-81 \%$ for prediction of the successful AVF maturation (42, 52).

Although preoperative assessment with DUS can lead to an increase in the number of AVFs created instead of AVGs or CVCs, AVF non-maturation rates still remain worrisome at present, preoperative assessment of single parameters does not enable adequate prediction of AVF function. Furthermore, the lack of standardized protocols limits the clinical value of the reported parameters. An 
important and impassible limitation of DUS is its shortcoming in evaluating calcified vessel segments or segments covered by bony structures (central thoracic, subclavian - and brachiochephalic arteries and veins for example).

\section{Digital subtraction angiography}

Digital subtraction angiography (DSA), using x-ray techniques and iodinated contrast-media is considered the standard of reference for assessment of upper extremity arteries and veins. The in-plane resolution as acquired with conventional or digital subtraction angiography is far superior to other imaging modalities like DUS or magnetic resonance angiography (MRA). A drawback however, is that acquired data are 2-dimensional (2D) projections of the complex three-dimensional (3D) vascular network in the upper extremity. This is an important limitation, especially in case of non-circular vessels and asymmetrical abnormalities that may not be appreciated in standard anterior-posterior and lateral projections. Therefore analysis is limited by the number and quality of the acquired projections. Furthermore, the clinical applicability in the work-up of endstage renal disease patients is limited due to the use of iodinated nephrotoxic contrast-media that may lead to temporary or permanent deterioration of residual renal function in up to $20 \%$ of the cases (63). Further deterioration of renal function should be avoided because loss of residual renal function is associated with higher morbidity and mortality rates $(64,65)$. Another important reason to avoid the use of iodinated contrast media is the chance of renal function recovery, even after initiation of hemodialysis therapy. In addition, residual kidney functions such as secretion of organic acids and various endocrine functions cannot be provided by dialysis and should therefore be preserved as long as possible (64). An additional obvious drawback of DSA is the invasive nature of the examination, especially for the depiction of the arterial system.

Geoffroy et al. reported on the safety and clinical performance of intra-arterial digital subtraction angiografphy (IA-DSA) using gadolinium chelates in ESRD patients and concluded that they were well tolerated with minimal impairment of renal function (mean $3 \%,<12 \%$ in all cases) (63). However, others reported serious complications associated with the off-label use of available gadolinium chelates at doses exceeding $0.3 \mathrm{mmol} / \mathrm{kg}$ (66-69). The high osmolality of contrast media is a pathogenetic factor in contrast induced nephropathy (CIN) 


\section{Chapter 2}

that potentially leads to further renal function impairment. CIN is more likely in patients with pre-existing impaired renal function (66, 67, 69, 70). Gadolinium chelates are hypertonic and the osmolality of commercially available gadolinium chelates is 2 to 7 times that of plasma $(66,69)$. To prevent CIN due to hypertonicity the dose should not exceed $0.3 \mathrm{mmol} / \mathrm{kg}$. Patients with older age, lower baseline creatinine clearance, diabetic nephropathy and low haemoglobin and albumin levels are at increased risk for developing gadolinium induced CIN, with a reported incidence between 0 and $11 \%(63,70)$.

Another alternative to iodinated contrast-media and gadolinium chelates is carbon dioxide $\left(\mathrm{CO}_{2}\right)$ gas injection (63). However, $\mathrm{CO}_{2}$ injections cause pain, and the $\mathrm{CO}_{2}$ contrast technique leads to stenosis grade overestimation and is therefore a less accurate method compared to IA-DSA and MRA (63). Other reasons to be prudent with the use of $\mathrm{CO}_{2}$ contrast are serious complications such as brain gas embolism during $\mathrm{CO}_{2}$ infusion for supradiaphragmatic arterial depiction and pulmonary embolism or acute cardiac arrest during upper extremity venography (63).

\section{Arterial assessment}

Upper extremity arteriography is traditionally performed by intra-arterial injection of contrast-media. Arterial access can be achieved by femoral or brachial puncture. In patients with ESRD the brachial artery approach is generally avoided because it can be painful, it may jeopardize distal perfusion and thereby maturation and function of a vascular access created distal to the puncture site. Drawbacks of arterial cannulation in general are puncture-related complications like hematoma, false aneurysms, dissection, infections and embolism with an overall incidence up to $11 \%(71-73)$. Thrombosis of the brachial artery is a serious complication due to brachial punctures for catheter access with a reported incidence of up to $7 \%$ (71-75). Contralateral venous injection of contrast-media is a less invasive alternative with fewer complications. However, in order to achieve sufficient arterial enhancement, this technique requires a high contrast dose which is not favorable in end-stage renal disease patients due to the potential further deterioration of residual renal function. 


\section{Venous assessment}

Central venous stenosis and obstruction occur frequently and often asymptomatic after central venous catheter insertion or placement of pacemaker leads. Imaging of the subclavian -, innominate - and superior caval veins prior to vascular access creation is important because $40 \%$ of patients with a history of central venous catheters have central venous stenosis or obstruction (76). Examples of central venous obstructions due to pacemaker wires and central venous catheter use are shown in figures 3 and 4.

Venography by cannulation of an ipsilateral dorsal hand vein allows imaging of the entire cephalic or basilic veins from the hand up to the confluence of the basilic and brachial veins into the subclavian vein. Although the superficial veins of the upper extremity are connected to each other at multiple levels, the puncture site
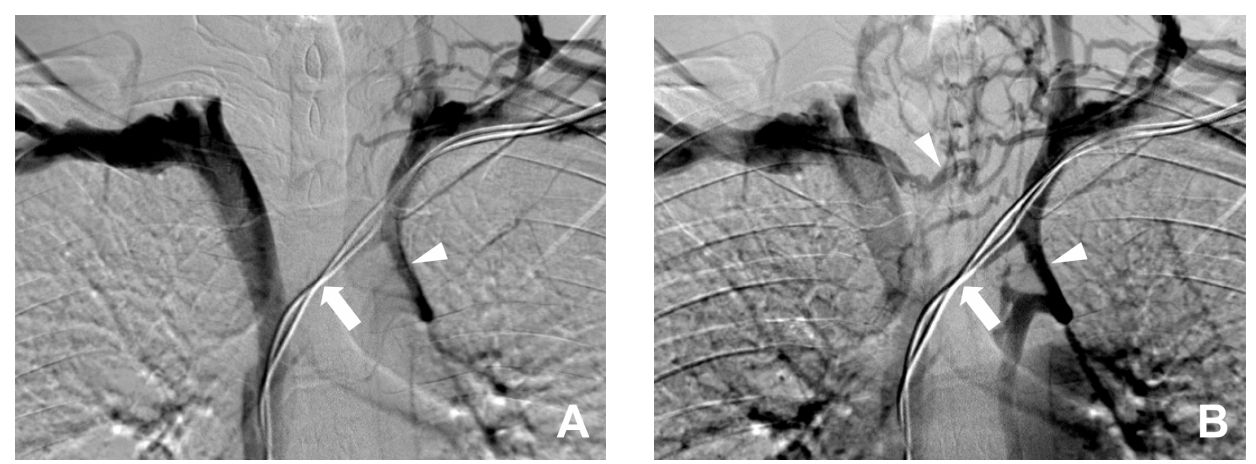

Figure 3: Two consecutive images obtained by digital subtraction angiography of a central venous obstruction (arrow) due to pacemaker wires. Arrowheads point at collateral draining veins.
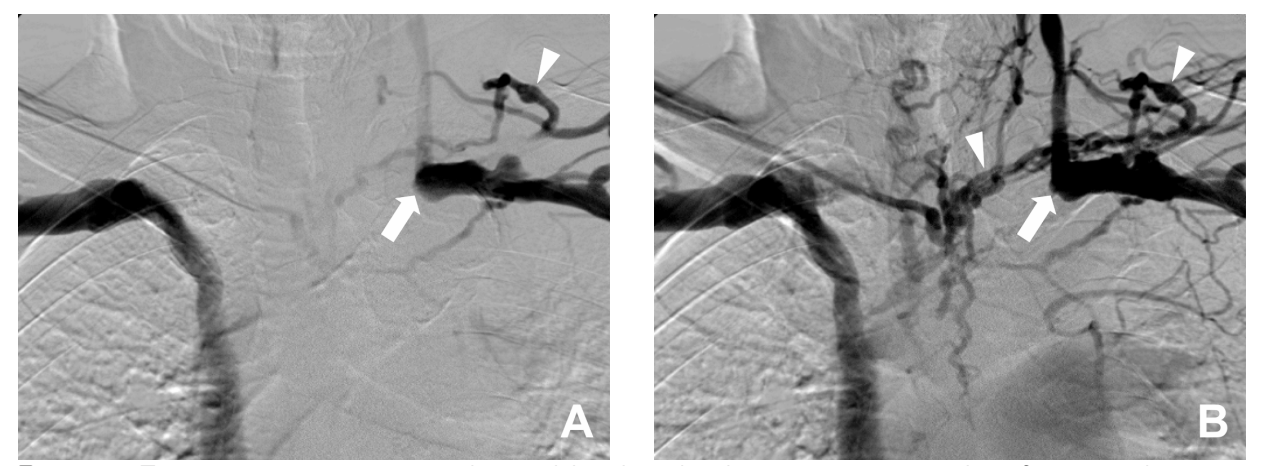

Figure 4: Two consecutive images obtained by digital subtraction angiography of a central venous obstruction (arrow) due to central venous catheter use. Arrowheads point at collateral draining veins. 
will limit venous opacification to the draining vein of the puncture site only. In our experience, the use of a proximal tourniquet inflated to $60 \mathrm{mmHg}$ enables depiction of collateral veins and improves assessment of venous diameter because of dilatation. Good inter-observer correlation coefficients have been reported for assessment of venous quality prior to access creation using conventional $\mathrm{X}$-ray venography techniques and gadolinium chelates as contrast media (kappa values, cephalic vein: forearm 0.65, upper arm 0.88; basilic vein: forearm 0.72; upper arm 0.64). Opacification of upper extremity veins is generally adequate although opacification of central veins can remain problematic, even after administration of increased contrast volumes (63).

\section{Magnetic resonance angiography}

Ongoing technical improvements have made magnetic resonance angiography (MRA) an important and valuable imaging modality in recent years in the preoperative workup of patients with ESRD. With the advent of contrastenhanced MRA techniques the role of non-enhanced time-of-flight (TOF) MRA has declined as it is prone to artifacts and stenoses are frequently overestimated (77). Because of this reason we only discuss contrast-enhanced MRA (CE-MRA).

CE-MRA protocols enable image acquisition with higher spatial (sub-millimeter voxel-size) and temporal resolutions ( $<20$ seconds per dynamic scan) with good to excellent image quality $(78,79)$. In CE-MRA, arterial and venous images are acquired in the coronal or sagittal plane during injection of contrast media. For arterial imaging $0.2-0.3 \mathrm{mmol} / \mathrm{kg} 0.5 \mathrm{M}$ extracellular gadolinium chelate contrast medium is injected in a contralateral antecubital or dorsal hand vein. For venous imaging, diluted $(1: 15)$ contrast media is injected in an ipsilateral dorsal hand vein.

Because intra-thoracic vessels are prone to movement during respiration, patients should hold their breath for about 15-20 seconds depending on the temporal and spatial resolutions and other technical factors related to system performance. Contrast medium is injected at speeds up to $3.0 \mathrm{~mL} / \mathrm{s}$, followed by $25 \mathrm{~mL}$ of saline flush. Spatial resolution in recent reports is typically in the order of $1.0 \times 1.0 \times 1.2 \mathrm{~mm}^{3}$ (cranio-caudal / frequency direction $\times$ left-right / phaseencoding directions $x$ antero-posterior / slice direction) (78). Using this approach for imaging, upper extremity arteries and veins can be visualized with high 

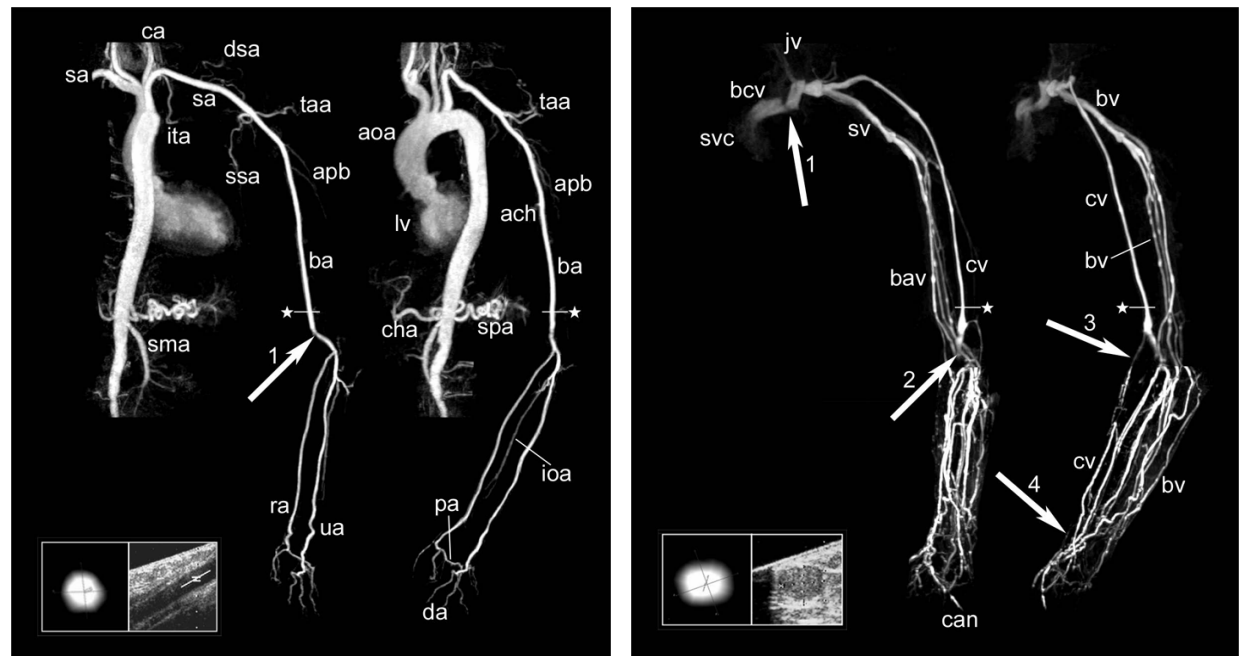

Figure 5: Example of selective CE-MRA of upper extremity arteries (left) and veins (right), by contralateral and ipsipateral injection of contrast media, respectively. Images of the entire upper extremity vasculature were acquired in four consecutive phases. First distal arteries (forearm), second proximal arteries (upper arm and thorax), third proximal veins (upper arm and thorax) and fourth distal veins (forearm). Legend: Iv = left ventricle, aa = aortic arch, sa = subclavian artery, $\mathrm{cca}=$ left common carotid artery, ita $=$ internal thoracic artery, dsa $=$ dorsal scapular artery, ataa $=$ acromial branch of the thoracoacromial artery, pbtaa $=$ posterior branch of the thoracoacromial artery, pba $=$ profunda brachial artery, ba $=$ brachial artery, aioa $=$ anterior interosseous artery, ra $=$ radial artery, ua $=$ ulnar artery, $\mathrm{pa}=$ palmar arch, da $=$ digital arteries, cha $=$ common hepatic artery, spa $=$ splenic artery, $\mathrm{sma}=$ superior mesenteric arteryLegend: $\mathrm{svc}=$ superior vena cava, $\mathrm{bcv}=$ brachiocephalic vein, $\mathrm{ijv}=$ internal jugular vein, $\mathrm{sv}=$ subclavian vein, axv = axillary vein, $\mathrm{cv}=$ cephalic vein, $\mathrm{bv}=$ brachial vein, bav = basilic vein, can = intravenous cannula for contrast media injection.

accuracy (78). Because the average upper extremity length of an adult is about $70-80 \mathrm{~cm}$, depicting the entire upper extremity requires imaging of at least 2 fields-of-view because of the limited MR-bore length. An example of a preoperative CE-MR arteriogram and venogram of an ESRD-patient is shown in figure 5 .

Synchronization of peak arterial or venous contrast concentration with sampling of central k-space profiles is very important in order to obtain selective imaging of arteries or veins, respectively (80). This is typically done by performing a timing sequence with 1-2 $\mathrm{mL}$ contrast medium prior to the contrast-enhanced acquisition, or by the use of real-time bolus monitoring software. With the latter technique the entire contrast bolus is injected and simultaneously monitored by either the operator or the MR scanner, and when sufficient enhancement is present in the target vessel, MR fluoroscopy sequence is aborted and the three- 


\section{Chapter 2}

dimensional CE-MRA acquisition is started. In order to improve both spatial and temporal resolution multi-element surface coils can be used to enable parallel imaging techniques (81). Apart from their parallel imaging capabilities these coils greatly improve vessel-to-background contrast.

\section{Arterial assessment}

Timing of contrast-media injection in relation to acquisition is crucial to achieve optimal vessel opacification in first pass arterial imaging. Wentz et al. reported good results using CE-MRA for imaging digital arteries using timed arterial compression to stop arterial flow and thereby enable longer scan time of a smaller field of view to allow increased spatial resolution without venous enhancement due to the required increase in scan times (79). Planken et al. reported a multi phase approach using multiple dynamic scans that resulted in good to excellent subjective image quality images of upper extremity arteries (78). However, assessment of the arterial palmar arch and digital arteries is cumbersome with the currently acquired spatial resolutions.

The introduction of blood pool contrast media enables higher resolution scans due to longer acquisition times for assessment of the palmar arch and digital arteries. In figure 6, an ultra high spatial resolution CE-MRA acquisition of the palmar arch and proximal digital arteries using a blood pool agent (Vasovist, Schering, Berlin, Germany) is shown.

CE-MRA is believed to be highly accurate for detection of arterial stenoses and obstructions. A typical example of a subclavian artery stenosis depicted by both CE-MRA and DSA is shown in figure 7. To date, however, no data about the accuracy and reproducibility for detection of thoracic and upper extremity arterial stenoses are available. 


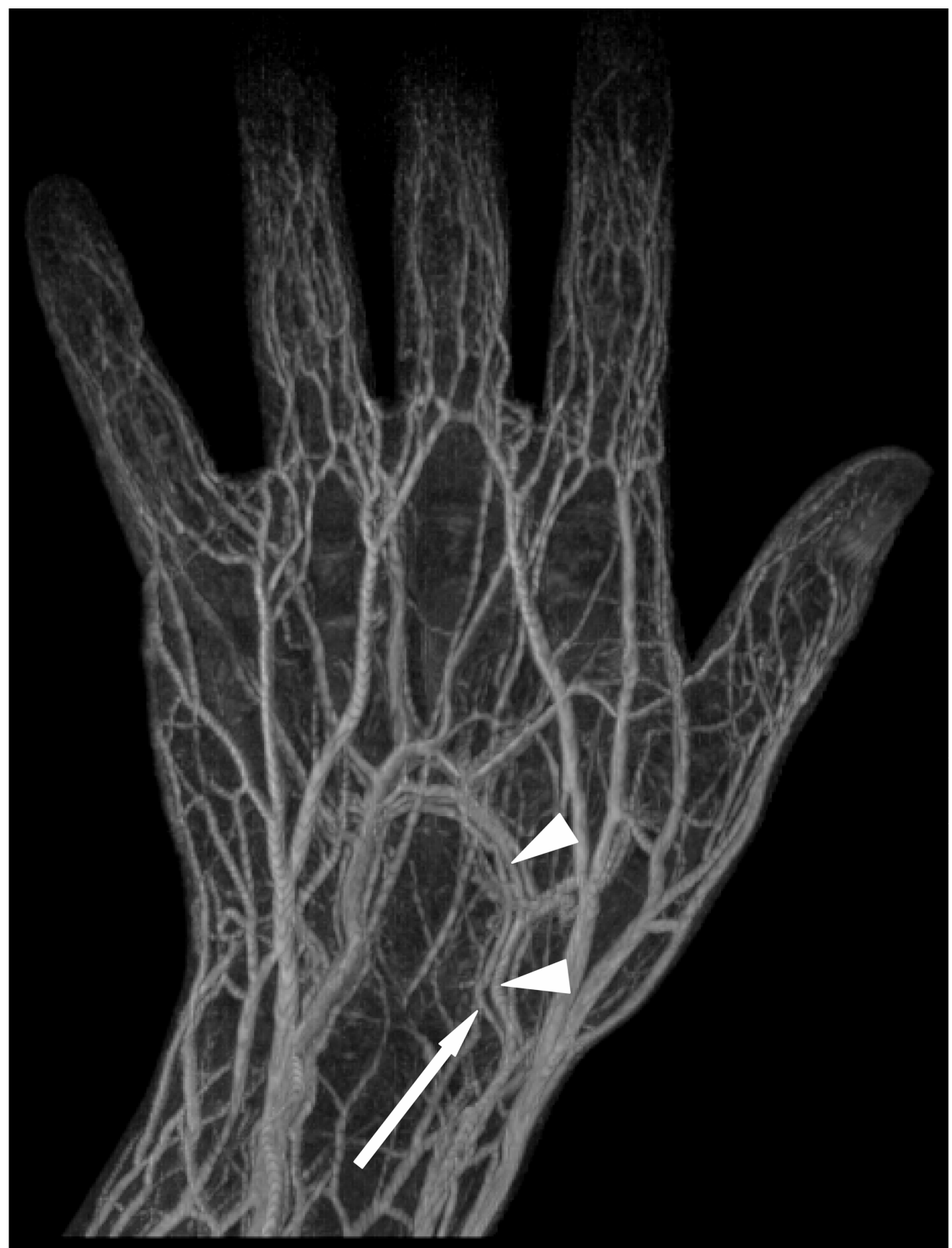

Figure 6: Ultra high resolution (acquired voxel size: $0.4 \times 0.4 \times 0.4 \mathrm{~mm}^{3}$ ) steady state CE-MRA of the arterial palmar arch and the proximal digital arteries, 45 seconds after injection of a blood pool agent. Although, both arteries and veins are equally opacified during steady state and separation of arteries and veins can be hazardous, the arterial palmar arch (arrowheads) can be identified as well as accompanying veins (arrow). 

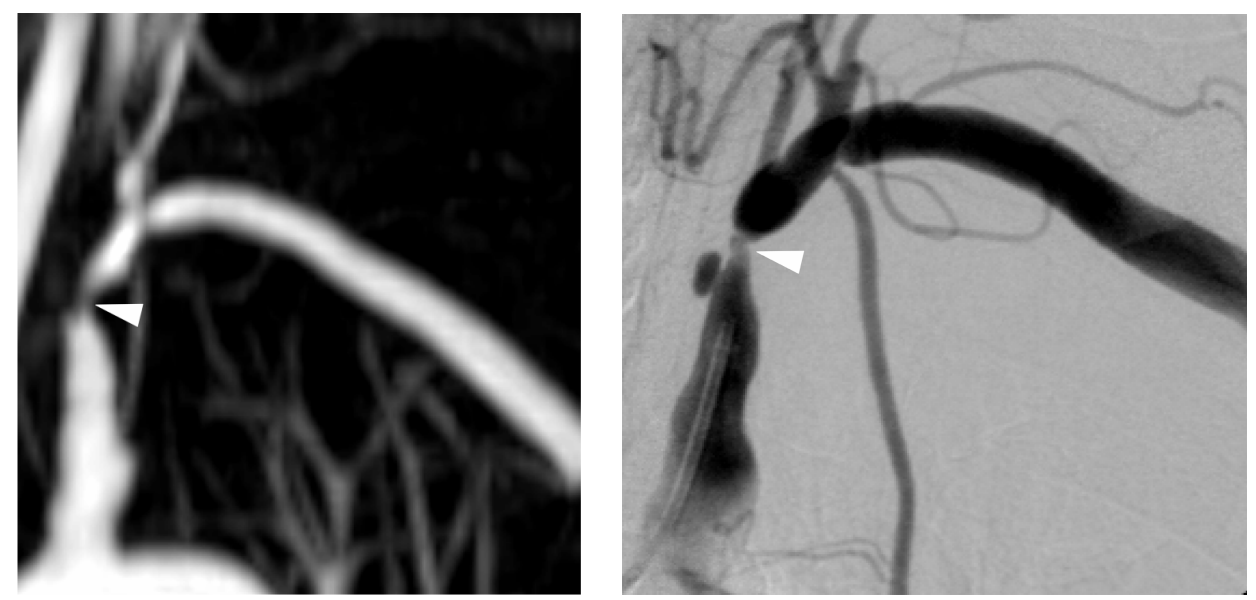

Figure 7: Typical example of a subclavian artery stenosis, initially detected by CE-MRA (left) and confirmed by DSA (right). The arrowhead indicates the location of the stenosis.

\section{Venous assessment}

Reported CE-MR venography techniques use either direct injection of diluted contrast-media ( $1: 15$ to $1: 25 \mathrm{~mL}$ of gadolinium chelate in saline solution) in the ipsilateral extremity or contralateral intravenous injection of non-diluted contrastmedia, and acquisition during delayed venous enhancement after initial arterial first pass (82-84). Both techniques have their strengths and weaknesses. Direct venography yields better vessel opacification with lower contrast dose as compared to the contralateral injection approach. Examples of a central venous stenosis and obstruction by direct MR venography are shown in figure 8 . However, direct injection without use of a proximal cuff may lead to selective opacification of veins in contrast to the contralateral injection approach that will result in opacification of all veins, albeit with much lower vessel to background contrast. On the other hand, timing is difficult when choosing the contralateral injection approach. To increase vessel to background contrast, subtraction techniques can be applied. However, application of subtraction techniques might, especially in central venous imaging, result in artifacts due to respiratory movements (84-87). Contrast-enhanced MR venography using delayed venous enhancement techniques showed poor performance for detection of central venous stenosis and occlusions (sensitivity and specificity $50 \%$ and $80 \%)(88,89)$. Direct CE-MR venography is easy to perform, well tolerated and highly accurate for detection of venous stenosis and obstructions in the 

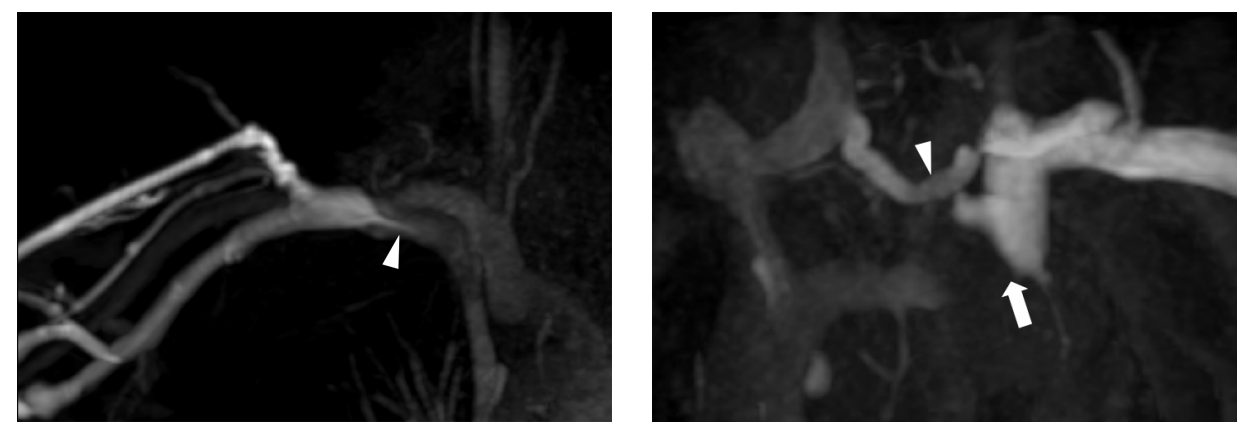

Figure 8 Example of a direct MR venogram of a patient with a central venous stenosis (left). The arrowhead points at the venous outflow stenosis. Example of a brachio-cephalic vein occlusion in another patient who had multiple central venous catheters (right). The arrow points at the obstructed brachio-cephalic vein in the direct CE-MR venogram. The arrowhead indicates a draining collateral vein.

upper extremity and central veins (82-84, 90-92). Furthermore, direct CE-MR venography diameter measurements are more accurate compared to duplex ultrasonography when using surgical measurements as standard of reference (78). Because of the lower contrast dose and better vessel opacification, direct CE-MR venography seems to be the method of first choice.

Although CE-MRA is a promising and attractive modality for imaging the upper extremity arteries and veins prior to vascular access creation, there are only sparse data about the clinical value in the work-up prior to vascular access creation. Future studies are needed to determine the clinical significance of adding CE-MRA to the preoperative work up prior to vascular access creation.

\section{Safety of Gadolinium containing contrast}

The most widely used gadolinium chelates for CE-MRA purposes are gadopentate dimeglumine (Magnevist, Schering, Berlin, Germany), gadoterate dimeglumine (Dotarem, Guerbet, Aulnay, France), gadodiamide (Omniscan, GE Health, Oslo, Norway) and gadoteridol (ProHance, Bracco Diagnostics, Milan, Italy). The total incidence of adverse events related to gadolinium use for CEMRA appears to be less than $5 \%$. The incidence of any single adverse event is approximately $1 \%$, or lower. By far the most common events are nausea, headache, and emesis (66). When used intravenously, no detectable nephrotoxicity has been reported and the rates of adverse events are extremely low (93-95). 
Recently concerns have arisen regarding the accumulation of free gadolinium in patients with renal failure (66). During the last decade approximately 200 cases of nephrogenic systemic fibrosis (NSF), previously known as nephrogenic fibrosing dermopathy, have been reported worldwide (96). The reported clinical signs and symptoms of NSF are subacute progressive swelling of extremities followed by more proximal involvement and sever skin induration, pain, muscle restlessness and loss of skin flexibility. NSF can lead to serious physical disability and wheelchair requirement (96). The incidence of NSF is low and the pathophysiology is unknown (96). To date, only gadolinium in the form of gadodiamide (Omniscan) has been reported to be associated with NSF.

Because of these reported adverse events and possible serious complication, prevention of dehydration and dialysis after gadodiamide injection are recommended as prophylactic measures.

\section{Summary and conclusions}

History taking and physical examination are cheap, readily available and sometimes valuable diagnostic tools to assess non-obese ESRD-patients prior to hemodialysis access creation. According to current guidelines, additional diagnostic modalities are indicated in case of suspected poor arterial or venous vessel quality, or in case of suspected central venous obstruction. The ultimate goal of preoperative assessment is to completely prevent non-maturation by optimal selection of the site of anastomosis and by identification and treatment of preexisting lesions before vascular access creation. However, even with the use of available diagnostic tools, non-maturation rates remain unacceptably high and standardized protocols for preoperative DUS, DSA or CE-MRA are lacking. Reported cut-off values for studied parameters are inconsistent which might be explained by differences between research groups in measurement protocols and differences in the parameters measured. The phenomenon of non-maturation remains poorly understood. To date, assessment of a single morphological or functional parameter has not enabled adequate prediction of postoperative AVF function for individual patients. To further unravel causes of AVF non-maturation more research is necessary. Furthermore, determination of measurement reproducibility for each parameter and each diagnostic modality is necessary before a standardized preoperative diagnostic approach can be implemented. 


\section{References}

1. Grassmann A, Gioberge S, Moeller S, Brown G. ESRD patients in 2004: global overview of patient numbers, treatment modalities and associated trends. Nephrol Dial Transplant 2005; 20:2587-2593.

2. Moeller S, Gioberge S, Brown G. ESRD patients in 2001: global overview of patients, treatment modalities and development trends. Nephrol Dial Transplant 2002; 17:2071-2076.

3. III. NKF-K/DOQI Clinical Practice Guidelines for Vascular Access: update 2000. Am J Kidney Dis $2001 ; 37: S 137-181$.

4. Clinical practice guidelines for vascular access. Am J Kidney Dis 2006; 48 Suppl 1:S176-247.

5. Anel RL, Yevzlin AS, Ivanovich P. Vascular access and patient outcomes in hemodialysis: questions answered in recent literature. Artif Organs 2003; 27:237-241.

6. Besarab A, Brouwer D. Improving arteriovenous fistula construction: Fistula firs initiative. Hemodialysis International 2004; 8:199-206.

7. Brescia MJ, Cimino JE, Appel K, Hurwich BJ. Chronic hemodialysis using venipuncture and a surgically created arteriovenous fistula. N Engl J Med 1966; 275:1089-1092.

8. Dhingra RK, Young EW, Hulbert-Shearon TE, Leavey SF, Port FK. Type of vascular access and mortality in U.S. hemodialysis patients. Kidney Int 2001; 60:1443-1451.

9. Harland RC. Placement of permanent vascular access devices: surgical considerations. Adv Ren Replace Ther 1994; 1:99-106.

10. Konner K, Nonnast-Daniel B, Ritz E. The arteriovenous fistula. J Am Soc Nephrol 2003; 14:1669-1680.

11. Lee H, Manns B, Taub K, et al. Cost analysis of ongoing care of patients with end-stage renal disease: the impact of dialysis modality and dialysis access. Am J Kidney Dis 2002; 40:611 -622.

12. Nassar GM, Ayus JC. Infectious complications of the hemodialysis access. Kidney Int 2001; 60:1-13.

13. Pisoni RL, Young EW, Dykstra DM, et al. Vascular access use in Europe and the United States: results from the DOPPS. Kidney Int 2002; 61:305-316.

14. Polkinghorne KR, McDonald SP, Atkins RC, Kerr PG. Vascular access and all-cause mortality: a propensity score analysis. J Am Soc Nephrol 2004; 15:477-486.

15. Rayner HC, Pisoni RL, Bommer J, et al. Mortality and hospitalization in haemodialysis patients in five European countries: results from the Dialysis Outcomes and Practice Patterns Study (DOPPS). Nephrol Dial Transplant 2004; 19:108-120.

16. Sands J, Perry M. Where are all the AV fistulas? Semin Dial 2002; 15:146-148.

17. Taylor G, Gravel D, Johnston L, Embil J, Holton D, Paton S. Prospective surveillance for primary bloodstream infections occurring in Canadian hemodialysis units. Infect Control Hosp Epidemiol 2002; 23:716-720. 


\section{Chapter 2}

18. Woods JD, Port FK. The impact of vascular access for haemodialysis on patient morbidity and mortality. Nephrol Dial Transplant 1997; 12:657-659.

19. Xue JL, Dahl D, Ebben JP, Collins AJ. The association of initial hemodialysis access type with mortality outcomes in elderly Medicare ESRD patients. Am J Kidney Dis 2003; 42:1013-1019.

20. Staramos DN, Lazarides MK, Tzilalis VD, Ekonomou CS, Simopoulos CE, Dayantas JN. Patency of autologous and prosthetic arteriovenous fistulas in elderly patients. Eur J Surg 2000; 166:777 781.

21. Kherlakian GM, Roedersheimer LR, Arbaugh JJ, Newmark KJ, King LR. Comparison of autogenous fistula versus expanded polytetrafluoroethylene graft fistula for angioaccess in hemodialysis. Am J Surg 1986; 152:238-243.

22. Leapman SB, Boyle M, Pescovitz MD, Milgrom ML, Jindal RM, Filo RS. The arteriovenous fistula for hemodialysis access: gold standard or archaic relic? Am Surg 1996; 62:652-656; discussion 656-657.

23. Tordoir JH, Mickley $\mathrm{V}$. European guidelines for vascular access: clinical algorithms on vascular access for haemodialysis. Edtna Erca J 2003; 29:131-136.

24. Rooijens PP, Tordoir JH, Stijnen T, Burgmans JP, Smet de AA, Yo TI. Radiocephalic wrist arteriovenous fistula for hemodialysis: meta-analysis indicates a high primary failure rate. Eur $\mathrm{J}$ Vasc Endovasc Surg 2004; 28:583-589.

25. Malovrh M. Native arteriovenous fistula: preoperative evaluation. Am J Kidney Dis 2002; 39:1218-1225.

26. Konner K, Hulbert-Shearon TE, Roys EC, Port FK. Tailoring the initial vascular access for dialysis patients. Kidney Int 2002; 62:329-338.

27. Malovrh M. Non-invasive evaluation of vessels by duplex sonography prior to construction of arteriovenous fistulas for haemodialysis. Nephrol Dial Transplant 1998; 13:125-129.

28. Mihmanli I, Besirli K, Kurugoglu S, et al. Cephalic vein and hemodialysis fistula: surgeon's observation versus color Doppler ultrasonographic findings. J Ultrasound Med 2001; 20:217222.

29. Zeebregts C, van den Dungen J, Bolt A, Franssen C, Verhoeven E, van Schilfgaarde R. Factors predictive of failure of Brescia-Cimino arteriovenous fistulas. Eur J Surg 2002; 168:29-36.

30. Turmel-Rodrigues L, Mouton A, Birmele B, et al. Salvage of immature forearm fistulas for haemodialysis by interventional radiology. Nephrol Dial Transplant 2001; 16:2365-2371.

31. Beathard GA, Arnold P, Jackson J, Litchfield T. Aggressive treatment of early fistula failure. Kidney Int 2003; 64:1487-1494.

32. Huber TS, Ozaki CK, Flynn TC, et al. Prospective validation of an algorithm to maximize native arteriovenous fistulae for chronic hemodialysis access. J Vasc Surg 2002; 36:452-459.

33. Allon M, Robbin ML. Increasing arteriovenous fistulas in hemodialysis patients: problems and solutions. Kidney Int 2002; 62:1109-1124. 
34. Miller PE, Tolwani A, Luscy $\mathrm{CP}$, et al. Predictors of adequacy of arteriovenous fistulas in hemodialysis patients. Kidney Int 1999; 56:275-280.

35. Patel ST, Hughes J, Mills JL, Sr. Failure of arteriovenous fistula maturation: an unintended consequence of exceeding dialysis outcome quality Initiative guidelines for hemodialysis access. J Vasc Surg 2003; 38:439-445; discussion 445.

36. Ascher E, Gade P, Hingorani A, et al. Changes in the practice of angioaccess surgery: impact of dialysis outcome and quality initiative recommendations. J Vasc Surg 2000; 31:84-92.

37. Tordoir JH, Rooyens P, Dammers R, van der Sande FM, de Haan M, Yo TI. Prospective evaluation of failure modes in autogenous radiocephalic wrist access for haemodialysis. Nephrol Dial Transplant 2003; 18:378-383.

38. Allen EV. Thromboangiitis obliterans: methods of diagnosis of chronic occlusive arterial lesions distal tho the wrist with illustrative cases. Am J Med Sci 1929; 2:1-8.

39. McGregor $A D$. The Allen test--an investigation of its accuracy by fluorescein angiography. J Hand Surg [Br] 1987; 12:82-85.

40. Ruengsakulrach P, Brooks M, Hare DL, Gordon I, Buxton BF. Preoperative assessment of hand circulation by means of Doppler ultrasonography and the modified Allen test. $J$ Thorac Cardiovasc Surg 2001; 121:526-531.

41. Vassalotti JA, Falk A, Cohl ED, Uribarri J, Teodorescu V. Obese and non-obese hemodialysis patients have a similar prevalence of functioning arteriovenous fistula using pre-operative vein mapping. Clin Nephrol 2002; 58:211-214.

42. Wong V, Ward R, Taylor J, Selvakumar S, How TV, Bakran A. Factors associated with early failure of arteriovenous fistulae for haemodialysis access. Eur J Vasc Endovasc Surg 1996; 12:207-213.

43. Katz ML, Comerota AJ, DeRojas J, Bowman G, Czeredarczuk M, White JV. B-mode imaging to determine the suitability of arm veins for primary arteriovenous fistulae. The Journal of Vascular Technology 1987; 11:172-174.

44. Allon M, Bailey R, Ballard R, et al. A multidisciplinary approach to hemodialysis access: prospective evaluation. Kidney Int 1998; 53:473-479.

45. Robbin ML, Gallichio MH, Deierhoi MH, Young CJ, Weber TM, Allon M. US vascular mapping before hemodialysis access placement. Radiology 2000; 217:83-88.

46. Silva MB, Jr., Hobson RW, 2nd, Pappas PJ, et al. A strategy for increasing use of autogenous hemodialysis access procedures: impact of preoperative noninvasive evaluation. J Vasc Surg 1998; 27:302-307; discussion 307-308.

47. Robbin ML, Chamberlain NE, Lockhart ME, et al. Hemodialysis arteriovenous fistula maturity: US evaluation. Radiology 2002; 225:59-64.

48. Lockhart ME, Robbin ML, Allon M. Preoperative sonographic radial artery evaluation and correlation with subsequent radiocephalic fistula outcome. J Ultrasound Med 2004; 23:161-168; quiz 169-171. 


\section{Chapter 2}

49. Wittenberg G, Landwehr P, Moll R, Tschammler A, Buschmann B, Krahe T. [Interobserver variability of dialysis shunt flow measurements using color coated duplex sonography]. Rofo 1993; 159:375-378.

50. Wittenberg G, Schindler T, Tschammler A, Kenn W, Hahn D. [Value of color-coded duplex ultrasound in evaluating arm blood vessels--arteries and hemodialysis shunts]. Ultraschall Med 1998; 19:22-27.

51. Mendes RR, Farber MA, Marston WA, Dinwiddie LC, Keagy BA, Burnham SJ. Prediction of wrist arteriovenous fistula maturation with preoperative vein mapping with ultrasonography. $J$ Vasc Surg 2002; 36:460-463.

52. Lemson MS, Leunissen KM, Tordoir JH. Does pre-operative duplex examination improve patency rates of Brescia-Cimino fistulas? Nephrol Dial Transplant 1998; 13:1360-1361.

53. Leblanc M, Saint-Sauveur E, Pichette V. Native arterio-venous fistula for hemodialysis: What to expect early after creation? The Journal of Vascular Access 2003; 4:39-44.

54. Trager S, Pignataro M, Anderson J, Kleinert JM. Color flow Doppler: imaging the upper extremity. J Hand Surg [Am] 1993; 18:621-625.

55. Yerdel MA, Kesenci M, Yazicioglu KM, Doseyen Z, Turkcapar AG, Anadol E. Effect of haemodynamic variables on surgically created arteriovenous fistula flow. Nephrol Dial Transplant 1997; 12:1684-1688.

56. Wall LP, Gasparis A, Callahan S, van Bemmelen P, Criado E, Ricotta J. Impaired hyperemic response is predictive of early access failure. Ann Vasc Surg 2004; 18:167-171.

57. Nack TL, Needleman L. Comparison of Duplex Ultrasound and Contrast Venography for Evaluation of Upper Extremity Venous Disease. The Journal Of Vascular Technology 1992; 16:69-73.

58. Brimble KS, Rabbat Ch G, Treleaven DJ, Ingram AJ. Utility of ultrasonographic venous assessment prior to forearm arteriovenous fistula creation. Clin Nephrol 2002; 58:122-127.

59. Lees TA, Manzo R, Strandness DE, Appleton D. Observer Variation in the Measurement of Venous Diameters Using Duplex Scanning. The Journal Of Vascular Technology 1994; 18:177180.

60. Planken RN, Keuter XH, Hoeks AP, et al. Diameter measurements of the forearm cephalic vein prior to vascular access creation in end-stage renal disease patients: graduated pressure cuff versus tourniquet vessel dilatation. Nephrol Dial Transplant 2006; $21: 802-806$.

61. Planken RN, Keuter XH, Kessels AG, Hoeks AP, Leiner T, Tordoir JH. Forearm cephalic vein cross-sectional area changes at incremental congestion pressures: towards a standardized and reproducible vein mapping protocol. J Vasc Surg 2006; 44:353-358.

62. Allon M, Lockhart ME, Lilly RZ, et al. Effect of preoperative sonographic mapping on vascular access outcomes in hemodialysis patients. Kidney Int 2001; 60:2013-2020.

63. Geoffroy O, Tassart M, Le Blanche AF, et al. Upper extremity digital subtraction venography with gadoterate meglumine before fistula creation for hemodialysis. Kidney Int 2001; 59:1491 1497. 
64. Jansen MA, Hart AA, Korevaar JC, Dekker FW, Boeschoten EW, Krediet RT. Predictors of the rate of decline of residual renal function in incident dialysis patients. Kidney Int 2002; 62:10461053.

65. Merkus MP, Jager KJ, Dekker FW, de Haan RJ, Boeschoten EW, Krediet RT. Predictors of poor outcome in chronic dialysis patients: The Netherlands Cooperative Study on the Adequacy of Dialysis. The NECOSAD Study Group. Am J Kidney Dis 2000; 35:69-79.

66. Shellock FG, Kanal E. Safety of magnetic resonance imaging contrast agents. J Magn Reson Imaging 1999; 10:477-484.

67. Sam AD, 2nd, Morasch MD, Collins J, Song G, Chen R, Pereles FS. Safety of gadolinium contrast angiography in patients with chronic renal insufficiency. J Vasc Surg 2003; 38:313-318.

68. Nyman U, Elmstahl B, Leander P, Nilsson M, Golman K, Almen T. Are gadolinium-based contrast media really safer than iodinated media for digital subtraction angiography in patients with azotemia? Radiology 2002; 223:311-318; discussion 328-319.

69. Nyman U, Golman K. Regarding "Safety of gadolinium contrast angiography in patients with chronic renal insufficiency". J Vasc Surg 2004; 40:204; author reply 204.

70. Ergun I, Keven K, Uruc I, et al. The safety of gadolinium in patients with stage 3 and 4 renal failure. Nephrol Dial Transplant 2006; 21:697-700.

71. Heenan SD, Grubnic S, Buckenham TM, Belli AM. Transbrachial arteriography: indications and complications. Clin Radiol 1996; 51:205-209.

72. Grollman JH, Jr., Marcus R. Transbrachial arteriography: techniques and complications. Cardiovasc Intervent Radiol 1988; 11:32-35.

73. Watkinson AF, Hartnell GG. Complications of direct brachial artery puncture for arteriography: a comparison of techniques. Clin Radiol 1991; 44:189-191.

74. Pepine CJ, Von Gunten C, Hill JA, et al. Percutaneous brachial catheterization using a modified sheath and new catheter system. Cathet Cardiovasc Diagn 1984; 10:637-642.

75. Rath J, Ganschow US, Kelm M, et al. [Duplex ultrasound risk stratification of percutaneous puncture of the brachial artery for diagnostic and interventional coronary angiography]. Z Kardiol 1998; 87:249-257.

76. Surratt RS, Picus D, Hicks ME, Darcy MD, Kleinhoffer M, Jendrisak M. The importance of preoperative evaluation of the subclavian vein in dialysis access planning. AJR Am J Roentgenol 1991; 156:623-625.

77. Meaney JF. Magnetic resonance angiography of the peripheral arteries: current status. Eur Radiol 2003; 13:836-852.

78. Planken RN, Tordoir JHM, de Haan MW, Backes WH, van Engelshoven JMA, Leiner T. Contrast Enhanced-MR Angiography of Upper Extremity Arteries and Veins Prior to Vascular Access Surgery. In: Mickley V, ed. 4th International Congress of the Vascular Access Society (VAS). Berlin: Karger, 2005; 227-261. 


\section{Chapter 2}

79. Wentz KU, Frohlich JM, von Weymarn C, Patak MA, Jenelten R, Zollikofer CL. High-resolution magnetic resonance angiography of hands with timed arterial compression (tac-MRA). Lancet 2003; 361:49-50.

80. Maki JH, Chenevert TL, Prince MR. Three-dimensional contrast-enhanced MR angiography. Top Magn Reson Imaging 1996; 8:322-344.

81. Weiger M, Pruessmann KP, Kassner A, et al. Contrast-enhanced 3D MRA using SENSE. J Magn Reson Imaging 2000; 12:671-677.

82. Ruehm SG. MR venography. Eur Radiol 2003; 13:229-230.

83. Ruehm SG, Zimny K, Debatin JF. Direct contrast-enhanced 3D MR venography. Eur Radiol $2001 ; 11: 102-112$.

84. Shinde TS, Lee VS, Rofsky NM, Krinsky GA, Weinreb JC. Three-dimensional gadoliniumenhanced MR venographic evaluation of patency of central veins in the thorax: initial experience. Radiology 1999; 213:555-560.

85. Kroencke TJ, Taupitz M, Arnold R, Fritsche L, Hamm B. Three-dimensional gadolinium-enhanced magnetic resonance venography in suspected thrombo-occlusive disease of the central chest veins. Chest 2001; 120:1570-1576.

86. Lebowitz JA, Rofsky NM, Krinsky GA, Weinreb JC. Gadolinium-enhanced body MR venography with subtraction technique. AJR Am J Roentgenol 1997; 169:755-758.

87. Oxtoby JW, Widjaja E, Gibson KM, Uzoka K. 3D gadolinium-enhanced MRI venography: evaluation of central chest veins and impact on patient management. Clin Radiol 2001; 56:887894.

88. Haire WD, Lynch TG, Lund GB, Lieberman RP, Edney JA. Limitations of magnetic resonance imaging and ultrasound-directed (duplex) scanning in the diagnosis of subclavian vein thrombosis. J Vasc Surg 1991; 13:391-397.

89. Baarslag HJ, Van Beek EJ, Reekers JA. Magnetic resonance venography in consecutive patients with suspected deep vein thrombosis of the upper extremity: initial experience. Acta Radiol 2004; 45:38-43.

90. Li W, David V, Kaplan R, Edelman RR. Three-dimensional low dose gadolinium-enhanced peripheral MR venography. J Magn Reson Imaging 1998; 8:630-633.

91. Goyen M, Barkhausen J, Kuehl H, et al. [Contrast-enhanced 3D MR venography of central thoracic veins: preliminary experience]. Rofo Fortschr Geb Rontgenstr Neuen Bildgeb Verfahr 2001; 173:356-361.

92. Thornton MJ, Ryan R, Varghese JC, Farrell MA, Lucey B, Lee MJ. A three-dimensional gadolinium-enhanced MR venography technique for imaging central veins. AJR Am J Roentgenol 1999; 173:999-1003.

93. Cochran ST, Bomyea K, Sayre JW. Trends in adverse events after IV administration of contrast media. AJR Am J Roentgenol 2001; 176:1385-1388.

94. Prince MR, Arnoldus C, Frisoli JK. Nephrotoxicity of high-dose gadolinium compared with iodinated contrast. J Magn Reson Imaging 1996; 6:162-166. 
95. Tombach B, Bremer C, Reimer $\mathrm{P}$, et al. Renal tolerance of a neutral gadolinium chelate (gadobutrol) in patients with chronic renal failure: results of a randomized study. Radiology 2001; 218:651-657.

96. Marckmann P, Skov L, Rossen K, et al. Nephrogenic systemic fibrosis: suspected causative role of gadodiamide used for contrast-enhanced magnetic resonance imaging. J Am Soc Nephrol 2006; 17:2359-2362. 



\section{Chapter 3}

Forearm cephalic vein cross-sectional area changes at incremental congestion pressures: towards a reproducible and standardized vein mapping protocol

R.N. Planken, X.H.A. Keuter, A.G.H. Kessels, A.P.G. Hoeks, T. Leiner, J.H.M. Tordoir

Journal of Vascular Surgery 2006: 44(2) 353-358 


\section{Abstract}

Objectives: Duplex ultrasonography (DUS) assessment of superficial forearm veins is frequently used prior to hemodialysis arteriovenous fistula (AVF) creation. However, there is no standardized preoperative DUS protocol. Purpose of this study was to assess B-mode image analysis reproducibility and reproducibility of repeated forearm superficial venous diameter measurements on different days at different venous congestion pressures (VCP).

Materials and methods: Diameters were determined using B-mode ultrasonography in 10 healthy male volunteers on days 1 and 14 at incremental VCP values ( 10 to $80 \mathrm{mmHg}$ ). The local medical ethics committee approved the study and all subjects were required to sign informed consent prior to inclusion in the study. Intra- and inter-observer agreement was assessed for B-mode image analysis by calculating interclass correlation coefficients (ICC). Reproducibility of repeated diameter measurements (maximum and minimum diameter at days 1 and 14), cross-sectional area (CSA) size increase and shape change due to incremental VCP were determined by calculating ICC values.

Results: Analysis of intra-observer agreement of B-mode image interpretation yielded ICC values of 0.97 ( $95 \% \mathrm{Cl} 0.94-0.99)$ and 0.97 (95\% Cl 0.96-0.99) for determination of maximum and minimum diameters, respectively. Inter-observer agreement analysis yielded ICC values of 0.95 (95\% Cl 0.92-0.97) and 0.96 (95\% $\mathrm{Cl}$ 0.96-0.99) for determination of maximum and minimum diameters, respectively. Reproducibility of repeated diameter measurements on days 1 and 14 improved substantially at incremental VCP values, with best reproducibility at VCP $>40 \mathrm{mmHg}$. Repeated determination of CSA size increase and shape change due to VCP increase from 10 to $80 \mathrm{mmHg}$ yielded ICC values of 0.49 (95\% Cl 0.19-1.00) and 0.09 (95\% Cl 0.00-0.92), respectively. Maximum and minimum diameters as well as CSA size increased significantly $(P<0.01)$ due to VCP increase during both sessions. CSA shape changed significantly $(P<0.01)$ due to VCP increase during both sessions.

Conclusions: Diameter measurements on B-mode images are largely observer independent. Superficial venous CSA shape is non-circular and CSA size depends on VCP. Both maximum and minimum venous diameters should be determined at VCP $>40 \mathrm{mmHg}$ to attain best reproducibility. Further studies are needed to determine whether a standardized preoperative vein mapping protocol can reduce AVF non-maturation rates. 


\section{Introduction}

Duplex ultrasonography (DUS) is frequently used for assessment of superficial arm veins prior to hemodialysis arteriovenous fistula (AVF) creation. At present, however, there is no standardized preoperative imaging protocol. Considering the persistently high AVF failure rates this is alarming (1). Accurate determination of venous diameter is important as this parameter has been demonstrated to correlate with long term AVF patency (2-6). Determination of venous diameter is not straightforward because superficial venous cross sectional area (CSA) is pressure dependent and non-circular in shape. This pressure dependence is infrequently mentioned but may have important consequences for preoperative diameter measurements.

Reported vein mapping protocols differ by venous congestion method, and the exact venous congestion pressure (VCP) used. Also the use of additional maneuvers (tapping, arm dangling, immersion in warm water, patient movement and positioning) to enable measurement of 'true' or 'maximum' venous diameter is inconsistent in literature $(7,8)$. Furthermore, there is no consensus on how exactly venous diameter should be measured: i.e. in anterior-posterior or medialto-lateral orientation. This is important because superficial venous cross sectional area (CSA) is not circular but elliptical in shape, even at higher VCP $(>65 \mathrm{mmHg})$ $(7,8)$. The CSA eccentricity can be expressed by the eccentricity ratio (ER) which is defined as the quotient of the maximum and minimum diameters at a given location. At present, the relationship between VCP and reproducibility of diameter measurements is unknown.

Another parameter used for preoperative prediction of vascular access function is forearm superficial venous diameter change over a range of different VCP (i.e. compliance) (3). To our knowledge, there are also no studies that have investigated the reproducibility of forearm superficial venous compliance measurements.

The purpose of the current study was to assess the reproducibility of B-mode image analysis and to assess the reproducibility of repeated forearm superficial venous diameter measurements on different days, and at different venous congestion pressures (VCP). 


\section{Chapter 3}

\section{Materials and methods}

\section{Subjects and study design}

Ten healthy male volunteers (mean age 26, range 25-28 years, mean BMI 22.4; range 18.6-26.8) participated in the study. None of the subjects had a reported history of previous intravenous accesses or indwelling catheter use. To determine method reproducibility, all subjects were assessed on days 1 and 14, at the same time of day, and under standardized conditions. All B-mode images were acquired by a single observer with two years experience in superficial vein mapping. Before each session, subjects were instructed to fast and abstain from exercise, nicotine, caffeine and alcohol in the 12 hours preceding the examination. The local medical ethics committee approved the study and all subjects were required to sign informed consent prior to inclusion in the study.

\section{Experimental setup and assessment of venous diameter}

A transverse B-mode image of the cephalic vein, $5 \mathrm{~cm}$ proximal to the radial head, was acquired using an Ultramark ultrasound scanner with a $38 \mathrm{~mm} 10 \mathrm{MHz}$ linear probe (HDI 9, ATL Inc., Bothel, WA) in each subject. All measurements were performed between 8:15 am and 11:00 am in a temperature-controlled room (range $22-24^{\circ} \mathrm{C}$ ). Total session time was 25 minutes for patient positioning and image acquisition. Subjects were in supine position with the arm next to the body at heart level. After a 15 minute acclimatization period, a proximal cuff for VCP application was inflated in incremental steps of $10 \mathrm{mmHg}$, each lasting 1 minute, and starting at $0 \mathrm{mmHg}$. Maximum applied VCP was $80 \mathrm{mmHg}$. One minute after each incremental VCP step, a B-mode image was recorded and stored in tagged image file format, resulting in 9 images per subject per session. Because resting intravenous pressure (at $\mathrm{VCP}=0 \mathrm{mmHg}$ ) is known to vary, these data were not included in the analysis (8).

Systolic, diastolic and mean upper extremity arterial blood pressures were recorded from the contralateral extremity (Dinamap Vital Signs Monitor 1846, Critikon Inc., Tampa, FL) to determine blood pressure differences between day 1 and 14 . 


\section{Image analysis}

\section{Reproducibility of B-mode image analysis}

Two observers, blinded for VCP and each other's results analyzed all B-mode images for each subject and each session in random order. For determination of intra-observer variation, the images were read again by the first observer two weeks after the first reading. To reduce recall bias in the evaluation, image order was scrambled in comparison to the first reading.

To determine venous CSA size and shape, all B-mode images were loaded into Matlab (Release 12.1, The MathWorks Inc., Natick, MA). Both observers measured the maximum and minimum diameters in each image. CSA area was subsequently calculated with the following formula:

$$
\mathrm{CSA}=\mathrm{D}_{1} * \mathrm{D}_{2} * \pi / 4
$$

(D1 = maximum diameter and D2 = minimum diameter)

\section{Reproducibility of repeated superficial venous measurements}

To determine intra-subject variation in venous diameters, CSA shape and CSA shape changes between different days, maximum diameter, minimum diameter and eccentricity ratios (ER) were calculated at each incremental VCP. Subsequently, these parameters were plotted as function of VCP values. Dynamic venous characteristics, i.e. percentage CSA size increase and ER decrease due to VCP increase from 10 to $80 \mathrm{mmHg}$, were determined for each subject on days 1 and 14, using the data obtained by observer 1 .

\section{Statistical analysis}

All statistical analyses were performed using SPSS statistical software for Windows (SPSS release 11.0.1, SPSS Inc, Chicago, IL) and STATA statistical software (STATA release 8, StataCorp LP, College Station, TX).

A paired sample Student's t-test was used to determine differences in systolic, diastolic and mean blood pressures between imaging sessions.

To assess intra- and inter-observer agreement of diameter measurements using B-mode images, interclass correlation coefficients were calculated on subject level for the findings on day 1 . In addition, reproducibility of repeated maximum 


\section{Chapter 3}

and minimum diameter measurements as well as CSA increase and ER decrease between days 1 and 14 were also determined by calculating ICC values. Confidence intervals for ICC values were computed using a bootstrap resampling technique with patient as resampling cluster (9). ICC values range between 0 and 1 , with values approaching 1 indicating stronger agreement among readers.

Differences in maximum and minimum diameters between VCP of 10 and 80 $\mathrm{mmHg}$ were assessed by a paired samples t-test. For all comparisons, the level of significance was set to $P<0.05$.

\section{Results}

All measurements were acquired successfully. There were no significant differences between sessions in contralateral upper arm arterial systolic (120.2 \pm 8.6 vs $117.3 \pm 9.4 \mathrm{mmHg})$, diastolic $(67.3 \pm 6.6$ vs $68.2 \pm 4.8 \mathrm{mmHg})$ or mean arterial pressures ( $85.5 \pm 7.6$ vs $84.2 \pm 6.4 \mathrm{mmHg}$ ) (all $P$-values $>0.05)$.

Reproducibility of $B$-mode image analysis

B-mode image interpretation was largely observer-independent. In table 1, ICC values of intra- and inter-observer agreement for determination of maximum and minimum venous diameters on B-mode images are listed.

\section{Reproducibility of repeated superficial venous measurements}

ICC values for repeated measurement of maximum and minimum diameter, and CSA size improved with increased VCP values. ICC values and 95\% confidence intervals for repeated ER determination, in contrast, initially improved until a VCP of $40 \mathrm{mmHg}$ but reproducibility deteriorated at VCP values $>40 \mathrm{mmHg}$. ICC values at incremental VCP values for repeated determination of maximum and minimum diameters, CSA and ER are shown in figure 1.

$\begin{array}{lll} & \text { Maximum diameter } & \text { Minimum diameter } \\ \text { Intraobserver agreement * } & 0.97(0.94-0.99) & 0.97(0.96-0.99) \\ \text { Interobserver agreement } & 0.95(0.92-0.97) & 0.96(0.96-0.99)\end{array}$

Table 1: Reproducibility of B-mode image analysis; Data are expressed as mean interclass correlation coefficient values and 95\% confidence intervals for intraobserver and interobserver agreement of Bmode image diameter measurements on day 1 (* for observer 1 ). 

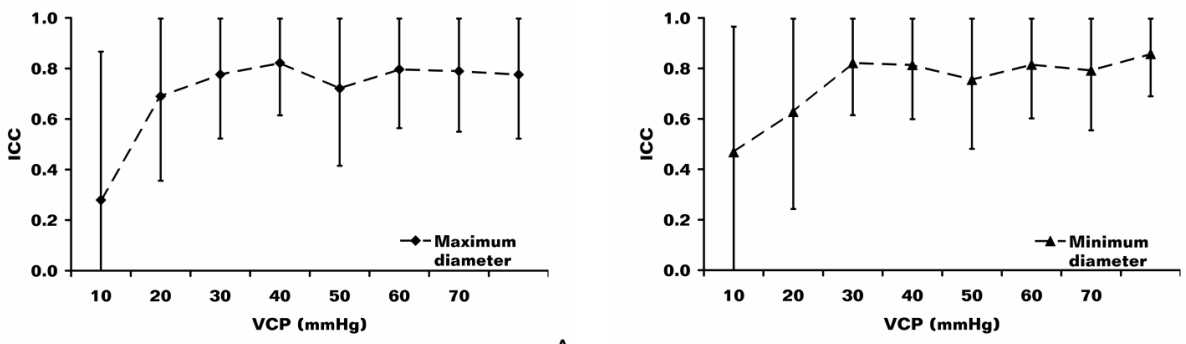

A

$\mathrm{B}$
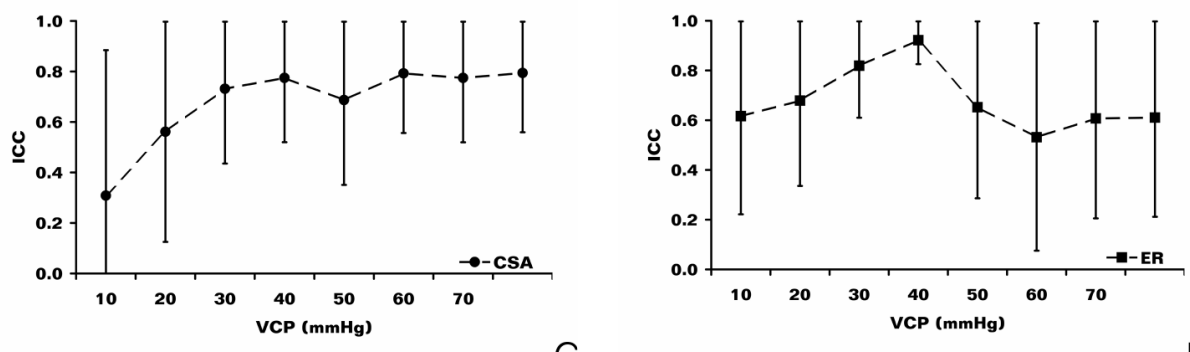

C

$\mathrm{D}$

Figure 1: Graph illustrating the relation between VCP and reproducibility of repeated determination of maximum diameter (A) minimum diameter (B), CSA size (C) and eccentricity ratio (ER) (D), respectively. Values represent mean ICC values and corresponding $95 \%$ confidence intervals as determined with the bootstrap technique.

Not surprisingly, increasing VCP led to larger maximum and minimum venous diameters, venous CSA and decreasing ER. A typical example of a series of transverse $B$-mode images of the cephalic vein at incremental VCPs is shown in figure 2.

Both maximum and minimum diameters increased significantly on days 1 and 14 (all $P$ values $<0.01$ ) due to VCP increase (maximum diameter day 1: from $3.5 \pm 0.9 \mathrm{~mm}$ to $4.3 \pm 1.2 \mathrm{~mm}$; day 14 : from $3.4 \pm 0.5 \mathrm{~mm}$ to $4.2 \pm 1.0 \mathrm{~mm}$; minimum diameter day $1: 1.9 \pm 0.5 \mathrm{~mm}$ to $3.3 \pm 0.7 \mathrm{~mm}$; day $14: 1.8 \pm 0.5 \mathrm{~mm}$ to $3.2 \pm 0.6 \mathrm{~mm})$. CSA also increased significantly $(P<0.01)$ from $5.5 \pm 2.4 \mathrm{~mm}^{2}$ to $11.7 \pm 5.1 \mathrm{~mm}^{2}$ on day 1 and from $4.9 \pm 1.7 \mathrm{~mm}^{2}$ to $10.9 \pm 4.4 \mathrm{~mm}^{2}$ on day 14 , due to VCP increase from 10 to $80 \mathrm{mmHg}$. The ER decreased significantly $(P<0.01)$ due to VCP increase (day $1: 1.9 \pm 0.3$ to $1.3 \pm 0.1$; day $14: 1.9 \pm 0.5$ to $1.3 \pm 0.2)$. Variations in maximum and minimum diameters, CSA, ER as function of VCP on days 1 and 14 are displayed in figure 3. 


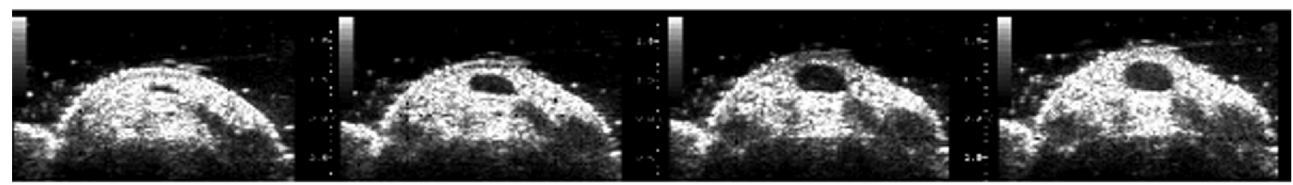

$10 \mathrm{mmHg}$

$20 \mathrm{mmHg}$

$30 \mathrm{mmHg}$

$40 \mathrm{mmHg}$

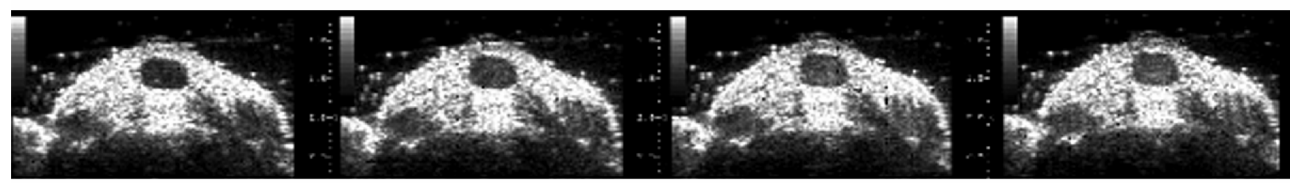

$50 \mathrm{mmHg}$

$60 \mathrm{mmHg}$

$70 \mathrm{mmHg}$

$80 \mathrm{mmHg}$

Figure 2: Example of a transverse B-mode depiction of the cephalic vein cross sectional area (CSA) at incremental venous congestion pressure (VCP). Note the CSA increase due to VCP increase is greatest in the pressure range between 10 and $40 \mathrm{mmHg}$.
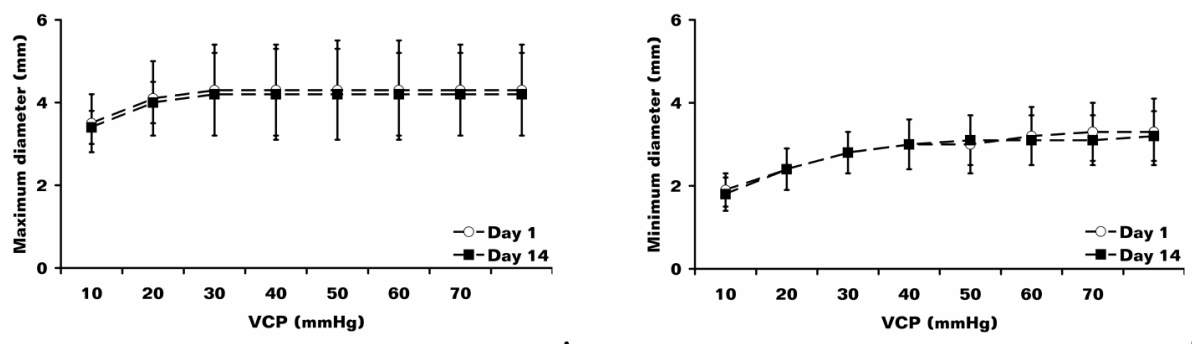

A
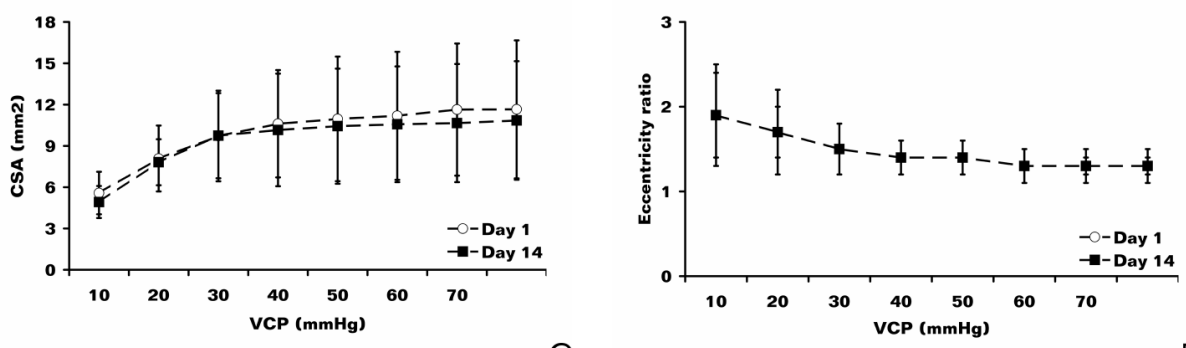

C

Figure 3: Graph illustrating the relation between VCP and maximum diameter (A) minimum diameter (B), CSA size (C) and eccentricity ratio (ER) (D), respectively. Values represent group means and standard deviations for days 1 and 14 .

Determination of CSA size increase and ER decrease at VCP increase from 10 $\mathrm{mmHg}$ to $80 \mathrm{mmHg}$ were poorly reproducible with ICC values of $0.49(95 \% \mathrm{CI}$ 0.19-1.00) and 0.09 (95\% Cl 0.00-0.92), respectively. 


\section{Discussion}

In the current study we found determination of venous diameters on B-mode ultrasonography images to be largely observer independent. Furthermore, we found that reproducibility of forearm superficial venous diameter measurement improved at higher VCPs. Surprisingly, the accuracy of ER determination progressively increased to a VCP of $40 \mathrm{mmHg}$ but declined again at higher VCPs.

We also found that forearm superficial venous maximum and minimum diameters as well as CSA size increased significantly due to an increase in VCP. Interestingly, forearm superficial venous shape remained elliptical at VCPs of up to $80 \mathrm{mmHg}$. CSA size increase and ER decrease between 10 and $80 \mathrm{mmHg}$ were poorly reproducible. The most likely reason for this finding is the poor reproducibility of the CSA size and ER at a VCP of $10 \mathrm{mmHg}$.

Preoperative measurement of forearm superficial venous diameter is important because this parameter has been reported to correlate with risk of nonmaturation of newly created $\operatorname{AVF}(1,6,10)$. Vein diameters smaller than 1.6 to $2.5 \mathrm{~mm}$ have been associated with AVF failure (2-5). However, reported cut-off diameters have been inconsistent and the exact cut-off diameter as well as the method of measurement remain subject of discussion as evidenced by high early failure and non-maturation rates (1-6, 10-13). The inconsistency in reported cutoff diameters might be due to differences in vein mapping protocols. Since the forearm superficial venous CSA remains elliptical, differences in reported cut-off diameters might also be due to differences in measurement orientation between research groups. Our results demonstrate that venous diameters vary substantially as a function of both the orientation at which they are measured, as well as the venous congestion pressure used. Furthermore, patient positioning and the type of venous congestion method used, also have a significant effect on superficial venous diameter and CSA shape, as was demonstrated by van Bemmelen et al. (7). Apart from variations in diameter arising from the use of different congestion methods and patient positioning there is additional variation over time. For instance, forearm superficial venous diameter has been reported to vary up to $27 \%$ between different days when assessed under standardized conditions and using a standardized vein mapping protocol (VCP $60 \mathrm{mmHg}$ ) (14). Future reports on the relation between forearm superficial venous diameter and 
AVF maturation should therefore specify exact measurement conditions and orientation.

De Groot et al. determined the reproducibility of CSA size increase due to VCP increase in deep conduit veins in the calf using B-mode imaging. The authors found variation coefficients ranging from 2 to $9 \%$ and 10 to $15 \%$ for the assessment of calf vein diameter and calf vein diameter increase due to VCP increase, respectively. As in the present study, measurement reproducibility improved at higher VCPs (15). However, it is well known that lower extremity veins differ from upper extremity veins but also that deep conduit veins differ from superficial veins in terms of anatomy, histology and function (8, 16-18). Unlike calf veins, superficial forearm veins play an important role in thermoregulation. Furthermore, superficial forearm veins remain elliptical at high VCPs in contrast to large conduit veins in the calf. The consequence of an elliptical CSA shape is increased venous resistance to blood flow when compared to a vein with a circular lumen and the same CSA size (19-21). The elliptical CSA shape might therefore have important clinical implications. However, whether differences in forearm superficial venous resistance due to differences in both CSA size and shape will affect AVF maturation and function needs to be assessed in future studies.

Superficial veins are highly compliant and are subject to caliber changes in response to VCP changes and environmental temperature fluctuations (8). In light of the findings in the current study, we believe static morphological parameters (e.g. diameter, CSA size or shape) only, are inadequate to characterize forearm superficial veins. This is corroborated by Malovrh et al. who reported that a low capacity of superficial veins to dilate due to VCP increase is associated with an increased risk of AVF non-maturation (diameter increase: $12 \%$ in failed AVF group versus $48 \%$ in success AVF group) (3). In the current study determination of CSA size increase and ER decrease due to VCP increase was poorly reproducible. This poor reproducibility can be attributed to the poor reproducibility of CSA size determination at low VCP values $(10 \mathrm{mmHg})$. An additional explanation for the poor reproducibility of these dynamic characteristics might be the vasomotion phenomenon, i.e. oscillatory changes in vessel diameters within minutes or daily variation in vascular tone (22). 
The current study has its limitations. First, the sample size was relatively small and only male volunteers were included. Females were not included to avoid bias due to differences in hormonal status between measurement sessions, which potentially affects both static and dynamic superficial venous characteristics. Inclusion of females would most likely have lead to decreased measurement reproducibility because of the menstrual cycle $(8,23)$. Because young healthy male volunteers were included and not end-stage renal disease patients, it is likely that reported mean diameters would have been smaller in the latter population. Because localization of the vessel wall on B-mode images is subject to measurement errors, measurement of small caliber vessels is likely to result in poorer measurement reproducibility compared to large caliber vessels because the measurement error will be a larger fraction of the measured diameter. Ultrasound systems with a better resolution might improve the reproducibility at low VCP, however a major source of error remains the biological variation.

Another limitation is that we did not image patients who were candidates for AVF placement. Measurement of superficial venous compliance is likely to be less reproducible in end-stage renal disease patients compared to young healthy male volunteers because these patients are known to have decreased venous compliance $(16,24,25)$. A comprehensive vascular evaluation prior to vascular access creation should include both venous and arterial assessment (26).

In conclusion, diameter measurements on B-mode images are largely observer independent. Superficial venous CSA shape is non-circular and CSA size depends on VCP. Both maximum and minimum venous diameters should be determined at a VCP $>40 \mathrm{mmHg}$ because of the much higher reproducibility. Further studies are needed to determine whether a standardized preoperative vein mapping protocol can reduce AVF non-maturation rates. 


\section{References}

1. Tordoir JH, Rooyens P, Dammers R, van der Sande FM, de Haan M, Yo TI. Prospective evaluation of failure modes in autogenous radiocephalic wrist access for haemodialysis. Nephrol Dial Transplant 2003; 18:378-383.

2. Silva MB, Jr., Hobson RW, 2nd, Pappas PJ, et al. A strategy for increasing use of autogenous hemodialysis access procedures: impact of preoperative noninvasive evaluation. J Vasc Surg 1998; 27:302-307; discussion 307-308.

3. Malovrh M. Native arteriovenous fistula: preoperative evaluation. Am J Kidney Dis 2002; 39:1218-1225.

4. Wong V, Ward R, Taylor J, Selvakumar S, How TV, Bakran A. Factors associated with early failure of arteriovenous fistulae for haemodialysis access. Eur J Vasc Endovasc Surg 1996; 12:207-213.

5. Ascher E, Gade P, Hingorani A, et al. Changes in the practice of angioaccess surgery: impact of dialysis outcome and quality initiative recommendations. J Vasc Surg 2000; 31:84-92.

6. Tordoir JH, Mickley V. European guidelines for vascular access: clinical algorithms on vascular access for haemodialysis. Edtna Erca J 2003; 29:131-136.

7. van Bemmelen PS, Kelly P, Blebea J. Improvement in the visualization of superficial arm veins being evaluated for access and bypass. J Vasc Surg 2005; 42:957-962.

8. Strandness DE, Sumner DS. Hemodynamics for Surgeons. New York: Grune \& Stratton, 1975.

9. Efron B TR. An introduction to the bootstrap: Chapman and Hall, London, 1993.

10. III. NKF-K/DOQI Clinical Practice Guidelines for Vascular Access: update 2000. Am J Kidney Dis 2001; 37:S137-181.

11. Allon M, Lockhart ME, Lilly RZ, et al. Effect of preoperative sonographic mapping on vascular access outcomes in hemodialysis patients. Kidney Int 2001; 60:2013-2020.

12. Malovrh M. Non-invasive evaluation of vessels by duplex sonography prior to construction of arteriovenous fistulas for haemodialysis. Nephrol Dial Transplant 1998; 13:125-129.

13. Robbin ML, Gallichio MH, Deierhoi MH, Young CJ, Weber TM, Allon M. US vascular mapping before hemodialysis access placement. Radiology 2000; 217:83-88.

14. Planken RN, Keuter XH, Hoeks AP, et al. Diameter measurements of the forearm cephalic vein prior to vascular access creation in end-stage renal disease patients: graduated pressure cuff versus tourniquet vessel dilatation. Nephrol Dial Transplant 2006; 21:802-806.

15. de Groot PC, Bleeker MW, Hopman MT. Ultrasound: a reproducible method to measure conduit vein compliance. J Appl Physiol 2005; 98:1878-1883.

16. Olsen $\mathrm{H}$, Lanne T. Reduced venous compliance in lower limbs of aging humans and its importance for capacitance function. Am J Physiol 1998; 275:H878-886. 
17. Schaper NC, Houben AJ, Schoon Y, Kooman JP, Huvers FC, Nieuwenhuijzen Kruseman AC. Venous compliance and the venodilatory effect of nitroglycerin in insulin-dependent diabetic patients with and without (incipient) nephropathy. Eur J Clin Invest 1994; 24:382-387.

18. Von Kugelgen $A$. [Relation of the circular musculature and internal pressure of the large veins in humans.]. Z Zellforsch Mikrosk Anat 1955; 43:168-183.

19. Li JK. Dynamics of the Vascular System: World Scientific Publishing Company, 2004.

20. Kresch $E$, Noordergraaf A. A mathematical model for the pressure-flow relationship in a segment of vein. IEEE Trans Biomed Eng 1969; 16:296-307.

21. Caro CG. The Mechanics of the Circulation: Oxford University Press, 1978.

22. Aalkaer $\mathrm{C}$, Nilsson H. Vasomotion: cellular background for the oscillator and for the synchronization of smooth muscle cells. Br J Pharmacol 2005; 144:605-616.

23. Meendering JR, Torgrimson BN, Houghton BL, Halliwill JR, Minson CT. Menstrual cycle and sex affect hemodynamic responses to combined orthostatic and heat stress. Am J Physiol Heart Circ Physiol 2005; 289:H631-642.

24. Kooman JP, Wijnen JA, Draaijer P, et al. Compliance and reactivity of the peripheral venous system in chronic intermittent hemodialysis. Kidney Int 1992; 41:1041-1048.

25. Lanne T, Olsen H. Decreased capacitance response with age in lower limbs of humans--a potential error in the study of cardiovascular reflexes in ageing. Acta Physiol Scand 1997; 161:503-507.

26. Konner K. When insufficient arterial inflow becomes the Achilles heel of the av-fistula-what are the surgical approaches? Nephrol Dial Transplant 2000; 15:145-147. 



\section{Chapter 4}

Diameter measurements of the forearm cephalic vein prior to vascular access creation in end-stage renal disease patients: graduated pressure cuff versus tourniquet vessel dilatation

R.N. Planken, X.H.A. Keuter, A.P.G. Hoeks, J.P. Kooman, F.M. van der Sande, A.G.H. Kessels, T. Leiner, J.H.M. Tordoir

Nephrology Dialysis Transplantation 2006: 21 (3) 802-806 


\section{Abstract}

Background: Preoperative assessment of forearm superficial venous diameter may predict early failure of newly created arteriovenous fistulas for hemodialysis access. However, early failure and non-maturation rates remain high (up to 30\%) and reported cut-off diameters are inconsistent. We hypothesize that this inconsistency is due to differences in the methods used to achieve venous dilatation prior to diameter measurements and daily variation in superficial venous diameter. We furthermore hypothesize that the use of a cuff will lead to a better inter-observer agreement since the applied pressure can be precisely determined. The purpose of this study was to determine inter-observer agreement of superficial venous diameter measurement under venous congestion by using either a graduated pressure cuff or tourniquet and furthermore to determine daily variations in superficial venous diameter.

Materials and methods: Diameter measurements were performed by two observers on days 1 and 3 , in 21 end-stage renal disease (ESRD) patients using either a cuff $(60 \mathrm{mmHg})$ or tourniquet. Measurements were carried out in random order and observers were blinded for each other's results. Interobserver agreement was expressed as interclass correlation coefficients. Variance components analysis was used to determine possible causes of disagreement.

Results: Using a cuff, mean venous diameter was $1.8 \mathrm{~mm}$ (range, 0.7-3.3 mm). When a tourniquet was used, the mean diameter was $1.8 \mathrm{~mm}$ (range, 0.6-3.2 $\mathrm{mm}$ ). Interclass correlation coefficients between observers were 0.76 and 0.74 for the use of a cuff and tourniquet, respectively. Diameter measurements revealed to be observer independent. Variations in venous diameter were determined by patient and the interaction of patient and day. Repeated assessment of venous diameter on different days revealed a variation coefficient of $26.4 \%$ when using a cuff, and $26.5 \%$ when using a tourniquet.

Conclusions: Venous diameter assessment is observer and congestion method independent. Daily variations in forearm superficial venous diameters should be taken into account when defining and using cut-off diameters prior to vascular access surgery. 


\section{Introduction}

Forearm superficial venous diameter has been shown to correlate with risk of non-maturation of newly created autogenous radial-cephalic arteriovenous fistulas for hemodialysis (RC-AVF) (1-3). Vein diameters smaller than 1.6 to 2.5 $\mathrm{mm}$ have been associated with RC-AVF failure (4-10). However, reported cut-off diameters are inconsistent and the exact cut-off diameter remains subject of discussion (4-10). This is underscored by the fact that early failure and nonmaturation rates remain up to $30 \%(1-3)$.

Because superficial forearm veins are highly compliant, local intravenous pressure is a major determinant of venous diameter (11). An inflatable pressure cuff or tourniquet at the upper arm is recommended to induce venous dilatation for better appreciation of maximum venous diameter $(1,2)$. The amount of pressure applied and thus the degree of venous dilatation cannot be determined exactly when using a tourniquet, in contrast to pressure application by a graduated inflatable cuff. We hypothesize that the use of a graduated inflatable pressure cuff will result in a better inter-observer agreement in upper extremity superficial venous diameter measurements compared to the use of a tourniquet. Daily variations in superficial venous diameter might limit the value of preoperative measured diameters to preoperatively predict AVF early failure and nonmaturation.

The purpose of the present study was to compare inter-observer agreement of venous diameter measurements when using either a graduated pressure cuff or a tourniquet. Furthermore, we assessed the variance in superficial forearm venous diameter over time as well as the influence of intrinsic patient factors.

\section{Materials and methods}

\section{Subjects and study design}

Twenty-one end-stage renal disease (ESRD) patients (mean age 63, range 28-87 years; mean body-mass index 24.4 , range $17.7-34.9)$ participated in the study. Causes of kidney failure were: diabetes type $2(n=4)$, hypertension $(n=3)$, nephrosclerosis $(n=3)$, nephrotic syndrome $(n=3)$, nephrolithiasis $(n=2)$, chronic pyelonephritis $(n=1)$, malignancy $(n=1)$ and of unknown origin $(n=4)$. 


\section{Chapter 4}

Thirteen patients were treated by peritoneal dialysis (mean creatinine clearance $12.8 \mathrm{~mL} / \mathrm{min}$; range $6.0-21.0 \mathrm{~mL} / \mathrm{min}$ ), and eight patients were awaiting vascular access creation for hemodialysis treatment purposes (mean creatinine clearance $14.9 \mathrm{~mL} / \mathrm{min}$; range $3.1-25.8 \mathrm{~mL} / \mathrm{min}$ ).

To determine method agreement for diameter measurements by using a cuff or tourniquet, all subjects were assessed twice, on days 1 and 3 , at the same time of day and under standardized conditions. Before each session, subjects were instructed to fast and abstain from exercise, nicotine, caffeine and alcohol in the 12-hours preceding the examination. The local medical ethics committee approved the study and all subjects were required to sign informed consent prior to inclusion in the study.

\section{Experimental setup and assessment of venous diameter}

B-mode images of the cephalic vein were acquired using an Ultramark ultrasound scanner with a $38 \mathrm{~mm}$ linear probe $5-10 \mathrm{MHz}$ (HDI 9, ATL Inc., Bothel, WA). All measurements were performed between 8:15 am and 11:00 am in a temperaturecontrolled room (range $22-24^{\circ} \mathrm{C}$ ). Total session time was 35 minutes for each subject, including 15 minutes of acclimatization.

To assess venous diameter, subjects were placed in supine position with the left arm placed in a fixation device (foam filled plastic vacuum bag), with the wrist at right atrium height. Special care was taken to avoid compression of the arm by the vacuum bag (figure 1). 


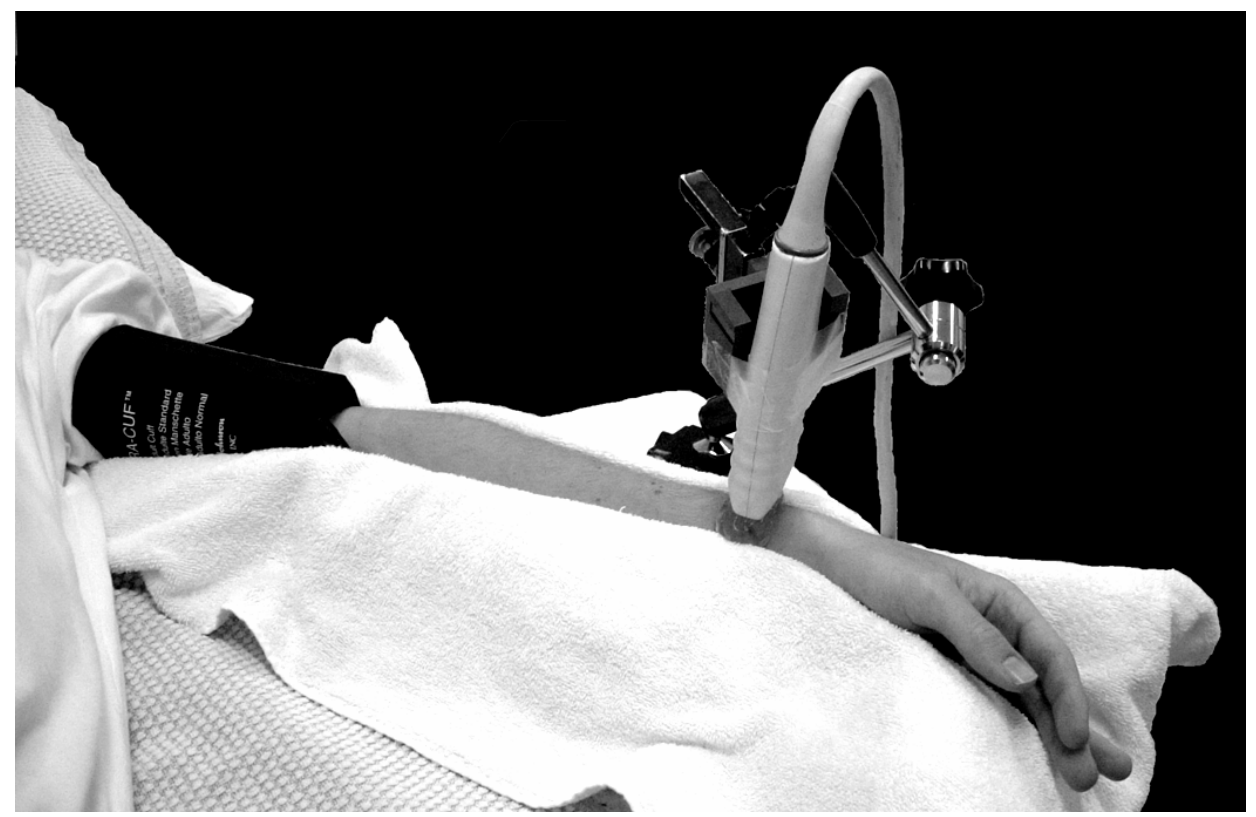

Figure 1: Positioning of forearm in fixation device. The ultrasound probe is fixed in a stand after optimal positioning for cross sectional imaging of the cephalic vein (see text).

After application of a proximal graduated pressure cuff (Iso Stabil 5, Speidel \& Keller $\mathrm{GmbH}$, Jungingen, Germany), inflated to $60 \mathrm{mmHg}$, the cephalic vein was localized and marked $5 \mathrm{~cm}$ proximal to the wrist joint. Subsequently, the cuff was deflated and a layer of at least $2 \mathrm{~cm}$ of gel was locally applied to optimize ultrasound image quality. The transducer was fixed in a stand perpendicular to the skin, after optimal positioning for cross-sectional imaging of the vein to enable diameter measurement perpendicular to the skin. Fixation of both the limb and the ultrasound probe avoided extremity or transducer displacement and axial movements. Measurements were started after a 15 minute acclimatization period following subject positioning. A proximal cuff (inflated to $60 \mathrm{mmHg}$ ) or tourniquet (manually adjusted) was applied at the upper arm. Two minutes after cuff or tourniquet application, the cross-sectional diameter of the cephalic vein was measured using a digital caliper with an accuracy of $0.1 \mathrm{~mm}$ on the ultrasonography system monitor.

All measurements were performed by two experienced observers who were blinded for their own and each others results. On day 1, both observers, measured venous diameter after application of either the cuff or tourniquet in 


\section{Chapter 4}

random order. Both cuff and tourniquet were applied by the observers themselves. After 15 minutes, measurements were repeated with the other method. In a second session two days later, both observers measured venous diameter again with both methods. Systolic, diastolic and mean arterial blood pressures were recorded from the contralateral upper extremity prior to acclimatization, directly before and directly after the measurements (Dinamap Vital Signs Monitor 1846, Critikon Inc., Tampa, FL), to determine variability of blood pressures over the course of the imaging sessions.

\section{Statistical analysis}

All statistical analyses were performed using SPSS statistical software for Windows (SPSS release 11.0.1, SPSS Inc, Chicago, IL). A paired sample Student's t-test was used to determine differences in systolic, diastolic and mean blood pressures between sessions. For all comparisons, the level of significance was set to $P<0.05$. To assess the inter-observer agreement, interclass correlation coefficients were calculated for diameter measurements by two observers. Variance components analysis was used to determine the effects of subject and observer for the variation in venous diameter measurements. The difference between the interclass correlation coefficients was tested with a bootstrap technique (12).

Mean differences and the standard deviation (SD) for venous diameters between days, when using a cuff by one observer, were determined for calculation of the variation coefficient for repeated diameter measurements at days 1 and 3 (= SD / Mean * 100\%) (13). Variation in venous diameter due to the used congestion method or day of measurement were visualized with Bland-Altman plots (14).

\section{Results}

All measurements were acquired successfully and there were no significant differences between sessions in contralateral upper arm arterial systolic, diastolic or mean arterial pressures (table 1). 

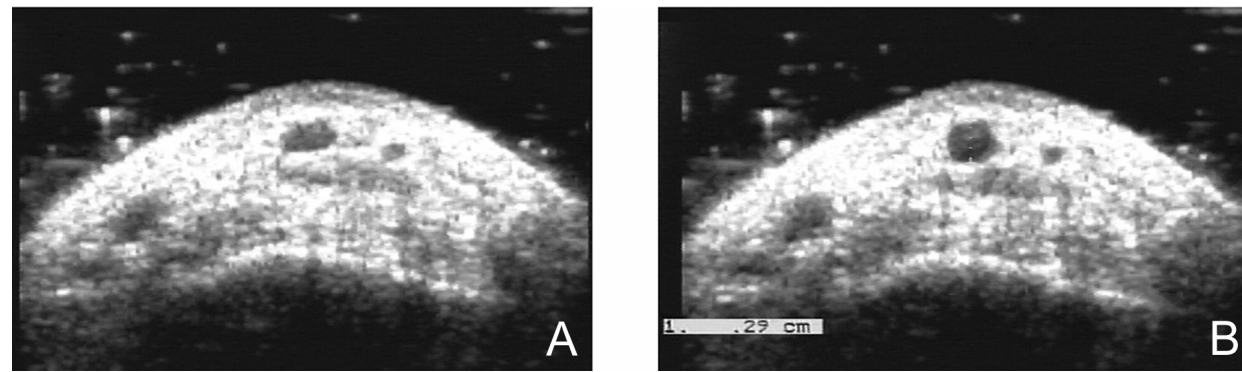

Figure 2: Cross sectional B-mode image of a cephalic vein prior to cuff inflation (A). In (B) the same location in the same vein is shown two minutes after the graduated pressure cuff has been inflated to $60 \mathrm{mmHg}$. The location of the cross-sectional depiction was identical for both images, about $5 \mathrm{~cm}$ proximal to the wrist joint. Note venous dilatation after application of the pressure cuff.

$\begin{array}{llcr} & \text { Day 1 } & \text { Day 3 } & \text { P-value } \\ \text { Systolic BP } & 138.3(20.2) & 139.6(20.4) & 0.62 \\ \text { Diastolic BP } & 75.5(11.2) & 77.5(11.6) & 0.31 \\ \text { Mean BP } & 101.5(15.9) & 101.6(14.7) & 0.97 \\ \text { Pulse rate } & 69.7(14.0) & 67.2(11.2) & 0.17\end{array}$

Table 1: Blood pressure and pulse rate for different sessions; Mean systolic -, diastolic and mean blood pressure (BP) and pulse rate for day 1 and day 3 . There is no significant difference between both sessions.

A typical example of a transverse $B$-mode image of the cephalic vein is shown in figure $2 \mathrm{~A}$ and $2 \mathrm{~B}$ before and after cuff application.

Using a graduated pressure cuff, mean venous diameter was $1.8 \mathrm{~mm}$ (range 0.7$3.3 \mathrm{~mm}$ ). When a tourniquet was used, the mean diameter was $1.8 \mathrm{~mm}$ (range 0.6-3.2 mm). Interclass correlation coefficients between observers were 0.76 and 0.74 for the use of a cuff and tourniquet, respectively. There were no significant differences between interclass correlation coefficients values of each method $(P>0.05)$. Venous diameter measurement on day 1 by observer 1 yielded a mean difference of $0.00 \mathrm{~mm}$ ( $S D=0.24 \mathrm{~mm}$ ) between methods (figure $3 A$ ). For observer 2 the mean difference was $0.02(S D=0.06 \mathrm{~mm})$.

Analysis of variance revealed that the patient was the main factor determining the variation in venous diameter measurements (variance $=0.303 \mathrm{~mm}^{2}$ ). Diameter measurements were almost observer independent (variance = $0.014 \mathrm{~mm}^{2}$ ). The factor day alone contributed negligible to total diameter variance (variance $=0.005 \mathrm{~mm}^{2}$ ). However, the diameter measurement revealed to be 


\section{Chapter 4}

dependent on interaction of subject and day (variance $=0.080 \mathrm{~mm}^{2}$ ). Repeated measurements of venous diameters on days 1 and 3 by one observer yielded a mean variation of $0.12(S D=0.48 \mathrm{~mm}$ ) (figure $3 B$ ).

Repeated assessment of venous diameter on different days revealed a variation coefficient of $26.4 \%$ when using a cuff, and $26.5 \%$ when using a tourniquet.
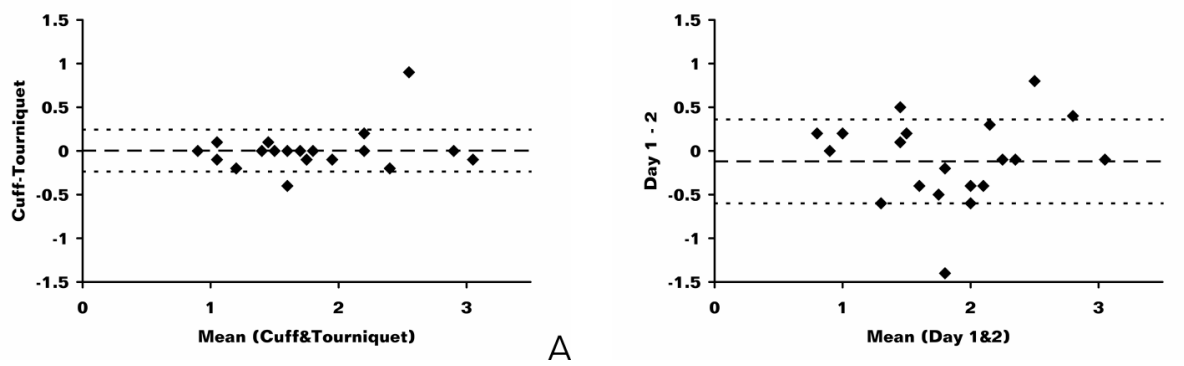

Figure 3: Bland-Altman plot showing differences in venous diameter measurement $(\mathrm{mm})$ between both congestion methods by one observer during one session $(n=21)(A)$. The dotted lines represent the mean value and standard deviation of the difference; (B): Bland-Altman plot showing difference in venous diameter measurement $(\mathrm{mm})$ using an inflatable cuff by observer 1 on day 1 versus day 3 $(n=21)$. The dotted lines represent the mean value and standard deviation of the difference

\section{Discussion}

In the current study we found reliability of forearm superficial venous diameter assessment to be independent of observer and congestion method used. The hypothesis that the use of a cuff will result in more accurate and precise superficial venous diameter measurements compared to the use of a tourniquet was not supported by our results. An additional source of variation might be inconsistency of tourniquet application. This is reflected by the difference between observers in the SD of the mean difference between the congestion methods used. Furthermore, our results indicate that forearm superficial venous diameters vary substantially between subjects and between days.

The comprehensive vascular evaluation prior to vascular access placement should also include evaluation of the arterial as well as the venous vasculature (15). However, reproducible measurement of superficial forearm venous diameter is crucial when choosing the type of access based on vessel diameters. Venous diameter measurement under congestion prior to vascular access surgery is 
recommended by the National Kidney Foundation (NKF DOQI guidelines) and the Vascular Access Society (Good Nephrological Practice guidelines) guideline on vascular access $(1,2)$. In these guidelines no preferred method has been specified.

Variance components estimation revealed that the factors subject and day determined variations in venous diameter measurements. There was, however, no systematic difference between the mean diameter measured on days 1 and 3 on group level. In contrast, variations at patient level were substantial, as evidenced by the variation coefficients of $26.4 \%$ and $26.5 \%$. Repeated measurements of venous diameters using a cuff yielded a SD of $0.5 \mathrm{~mm}$ which seems to be clinically inadequate, taken into account that the clinically used cut-off diameters range from $>1.6$ to $>2.5 \mathrm{~mm}$ (4-10). Furthermore, the variation of venous diameter over the course of time in this study might even be an underestimation of the true venous diameter variation because a potential recall bias can not be excluded.

Day-to-day variations in venous diameter might have been due to differences in venous dilatation due to alterations in vascular tone. However, disturbing factors modifying the sympathetic nervous tone and venous diameter (temperature, physical activity, mental state, alcohol, nicotine, caffeine etc), were controlled for. Sympathetic tone itself, however, was not controlled and this might have affected day-to-day variation on patient level. Furthermore, a venous diameter is a static parameter which is used for characterization of dynamic tissue (e.g. the cephalic vein). A dynamic measure might be more suitable for this purpose as has been demonstrated by Malovrh et al. who found that cephalic vein compliance (i.e. venous diameter increase due to congestion pressure increase) rather than diameter is correlated with RC-AVF non-maturation $(8,10)$. In the subgroup of patients in whom the RC-AVF failed a $48 \%$ lower diameter increase due to venous congestion was found when compared to the group in whom the RC-AVF was functioning well (10).

In conclusion, venous diameter assessment is observer and congestion method independent in this study setup. Daily variations in forearm superficial venous diameters should be taken into account when defining and using cut-off diameters prior to vascular access surgery. 


\section{Chapter 4}

\section{References}

1. III. NKF-K/DOQI Clinical Practice Guidelines for Vascular Access: update 2000. Am J Kidney Dis 2001; 37:S137-181.

2. Tordoir JH, Mickley V. European guidelines for vascular access: clinical algorithms on vascular access for haemodialysis. Edtna Erca J 2003; 29:131-136.

3. Tordoir JH, Rooyens P, Dammers R, van der Sande FM, de Haan M, Yo TI. Prospective evaluation of failure modes in autogenous radiocephalic wrist access for haemodialysis. Nephrol Dial Transplant 2003; 18:378-383.

4. Allon M, Lockhart ME, Lilly RZ, et al. Effect of preoperative sonographic mapping on vascular access outcomes in hemodialysis patients. Kidney Int 2001; 60:2013-2020.

5. Ascher E, Gade P, Hingorani A, et al. Changes in the practice of angioaccess surgery: impact of dialysis outcome and quality initiative recommendations. J Vasc Surg 2000; 31:84-92.

6. Silva MB, Jr., Hobson RW, 2nd, Pappas PJ, et al. A strategy for increasing use of autogenous hemodialysis access procedures: impact of preoperative noninvasive evaluation. J Vasc Surg 1998; 27:302-307; discussion 307-308.

7. Wong V, Ward R, Taylor J, Selvakumar S, How TV, Bakran A. Factors associated with early failure of arteriovenous fistulae for haemodialysis access. Eur J Vasc Endovasc Surg 1996; 12:207-213.

8. Malovrh M. Non-invasive evaluation of vessels by duplex sonography prior to construction of arteriovenous fistulas for haemodialysis. Nephrol Dial Transplant 1998; 13:125-129.

9. Robbin ML, Gallichio MH, Deierhoi MH, Young CJ, Weber TM, Allon M. US vascular mapping before hemodialysis access placement. Radiology 2000; 217:83-88.

10. Malovrh M. Native arteriovenous fistula: preoperative evaluation. Am J Kidney Dis 2002; 39:1218-1225.

11. Strandness DE, Sumner DS. Hemodynamics for Surgeons. New York: Grune \& Stratton, 1975.

12. Efron B TR. An introduction to the bootstrap: Chapman and Hall, London, 1993.

13. Bland JM. An introduction to medical statistics. Oxford: Oxford University Press, 1987.

14. Bland JM, Altman DG. Statistical methods for assessing agreement between two methods of clinical measurement. Lancet 1986; 1:307-310.

15. Konner K. When insufficient arterial inflow becomes the Achilles heel of the av-fistula-what are the surgical approaches? Nephrol Dial Transplant 2000; 15:145-147. 




\section{Chapter 5}

Magnetic resonance angiographic assessment of upper extremity vessels prior to vascular access surgery: feasibility and accuracy

R.N. Planken, J.H.M. Tordoir, L.E.M. Duijm, H.C. van den Bosch, F.M. van der Sande, J.P. Kooman, M.W. de Haan, T. Leiner

European Radiology: in press 


\section{Abstract}

Purpose: To develop a contrast-enhanced magnetic resonance angiography (CEMRA) protocol for selective imaging of the entire upper extremity arterial and venous tree in a single exam.

Materials and Methods: 25 end-stage renal disease (ESRD) patients underwent CE-MRA and duplex ultrasonography (DUS) of the upper extremity prior to hemodialysis vascular access creation. Accuracy of CE-MRA arterial and venous diameter measurements were compared with DUS and intra-operative (IO) diameter measurements, the standard of reference.

Results: Upper extremity vasculature depiction was feasible with CE-MRA. CEMRA forearm and upper arm arterial diameters were $2.94 \pm 0.67 \mathrm{~mm}$ and 4.05 $\pm 0.84 \mathrm{~mm}$, respectively. DUS arterial diameters were $2.80 \pm 0.48 \mathrm{~mm}$ and 4.38 $\pm 1.24 \mathrm{~mm} ; \mathrm{IO}$ diameters were $3.00 \pm 0.35 \mathrm{~mm}$ and $3.55 \pm 0.51 \mathrm{~mm}$. Forearm arterial diameters were accurately determined with both techniques. Both techniques overestimated upper arm arterial diameters significantly. Venous diameters were accurately determined with CE-MRA but not with DUS (Forearm: CE-MRA: $2.64 \pm 0.61 \mathrm{~mm}$; DUS: $2.50 \pm 0.44 \mathrm{~mm}$, and 10 : 3.40 $\pm 0.22 \mathrm{~mm}$; upper arm: CE-MRA: $4.09 \pm 0.71 \mathrm{~mm}$; DUS: $3.02 \pm 1.65 \mathrm{~mm}$, and $1 O$ : $4.30 \pm 0.78 \mathrm{~mm})$.

Conclusion: CE-MRA enables selective imaging of upper extremity vasculature in patients requiring hemodialysis access. Forearm arterial diameters can be assessed accurately by CE-MRA. Both CE-MRA and DUS slightly underestimate upper arm arterial diameters. In comparison to DUS, CE-MRA enables a more accurate determination of upper extremity venous diameters. 


\section{Introduction}

The number of patients requiring hemodialysis because of end-stage renal disease (ESRD) is rising rapidly. In 2004, the number of ESRD patients was $1,783,000$ world wide and the majority of patients was being treated by hemodialysis $(1,2)$. An important problem in hemodialysis patients is nonmaturation of surgically created arteriovenous fistulae (AVF). Non-maturation refers to a lack of postoperative diameter and flow increase after initial creation and, ultimately, shunt failure (3). Although the exact mechanism of early failure and non-maturation remains elusive, it is widely recognized that preoperative assessment of upper extremity vessels helps to avoid non-maturation and contributes to ensuring long term patency of $\operatorname{AVF}(4,5)$. Prior to $\operatorname{AVF}$ creation possible access configurations and the adequacy of arterial inflow and venous outflow are therefore carefully studied. In terms of access type, AVF are preferred over arteriovenous grafts (AVG) because of long-term patency and lower complication rates.

Because of its widespread availability and noninvasive character, duplex ultrasonography (DUS) is currently the most widely used tool to assess the patency and diameter of peripheral arterial and venous sites prior to AVF placement $(4,5)$. Preoperative diameters of both arteries and veins of less than 1.6-2.5 $\mathrm{mm}$ have been have been associated with early AVF failure and nonmaturation of newly created AVFs (6-16). However, even with the use of preoperative DUS, reported early failure rates of autogenous AVFs remain up to $50 \%(6,13,16-22)$. One possible explanation might be the poor performance of DUS for assessment of arterial and venous diameter measurements. Another possible explanation for this persistently high failure rate is the known poor performance of DUS for the assessment of central arteries and veins due to the presence of disturbing bony structures. This is reflected in the finding that preoperative DUS examination of both arteries and veins has a sensitivity and specificity, positive predictive value and negative predictive value of $20-44 \%$, $94-100 \%, 60-100 \%$ and $73-81 \%$ for prediction of successful postoperative AVF function respectively $(14,23)$.

The poor clinical performance of DUS has created the need for additional preoperative diagnostic tools to help avoid AVF early failure and non-maturation. 
A promising imaging modality for the depiction of upper extremity arterial and venous anatomy and possible alternative to DUS is contrast-enhanced magnetic resonance angiography (CE-MRA). This technique potentially allows for rapid arterial and venous mapping and in contrast to DUS, enables true threedimensional imaging over a large field of view.

The objective of the present study was to develop and assess the feasibility of a new CE-MRA protocol for selective imaging of the entire upper extremity arterial and venous tree in a single exam in patients requiring hemodialysis vascular access. Furthermore, we compared the accuracy of preoperative arterial and venous diameter measurements using CE-MRA and DUS images. Intra-operative (IO) diameter measurements served as the standard of reference.

\section{Materials and methods}

A total of 25 consecutive patients with ESRD ( 18 male, 7 female, mean age: 65.9 \pm 15.8 years, range: $34-90$ ) scheduled for hemodialysis access creation were included. All patients underwent DUS and CE-MRA assessment prior to surgical AVF creation. The time between CE-MRA and surgery was 4 days (range: 1-10) and for DUS and surgery 23 days (range 1-30). The medical ethics committee of the institution approved the study and all patients signed informed consent prior to inclusion.

\section{MR imaging}

For all acquisitions, a commercially available 1.5-T MR imager (Intera R9.1, Philips Medical Systems, Best, The Netherlands) was used with a gradient strength of $23 \mathrm{mT} / \mathrm{m}$ and maximum slew rate of $115 \mathrm{mT} / \mathrm{m} / \mathrm{msec}$. For signal transmission, the built-in quadrature body coil was used. Prior to imaging, 20gauge venous access (Venflon; Ohmeda, Helsingborg, Sweden) was established in dorsal hand veins on both sides. The contra- and ipsilateral venous access were used for arterial and venous imaging, respectively.

Patients were placed head first in the bore of the MR scanner in the supine position with the upper extremities positioned at the patient's side. The imaging protocol consisted of imaging the distal and proximal upper extremity in four separate acquisitions. First, arteries of the distal upper extremity (lower arterial station, phase 1) were imaged, followed by the proximal upper extremity and 
chest arteries (upper arterial station, phase 2). Thereafter, images of the chest and proximal upper extremity veins (upper venous station, phase 3 ) and distal upper extremity veins (lower venous station, phase 4) were obtained. The proximal acquisitions consisted of two dynamic phases each; the distal acquisitions comprised three phases.

To prescribe the imaging volumes for the 3D CE-MRA acquisitions, non-enhanced time-of-flight (TOF) images were acquired in the proximal and distal stations. Parameters for TOF sequence were: $4.4 / 2.0$ (TR [msec] / TE [msec]), a 50 flip angle, a $500 \times 100 \mathrm{~mm}$ field of view, a $512 \times 192$ matrix scan, 25 transverse sections of $10-\mathrm{mm}$ thickness and a $10-\mathrm{mm}$ intersection gap. No saturation bands were used in order to depict both arteries and veins. The total imaging time for each station with this sequence was 62 seconds. Subsequently the 3D CE-MRA volumes were tailored to individual patient anatomy in both stations, using orthogonal maximum intensity projections as well as source images of the TOF acquisitions. For all 3D CE-MRA acquisitions, a gradient-recalled-echo (GRE) pulse sequence was used; the imaging parameters used are listed in table 1.

To reduce motion artifacts breath hold commands were given when imaging arteries and veins in the proximal station. For signal reception a 4-element quadrature phased array surface coil (Sense-Body, Philips Medical Systems) and a 2-element phased array surface coil (Synergy-Flex-L, Philips Medical Systems), were used for imaging the upper and lower stations, respectively. The surface coils were positioned as shown in figure 1 . 


\section{Chapter 5}

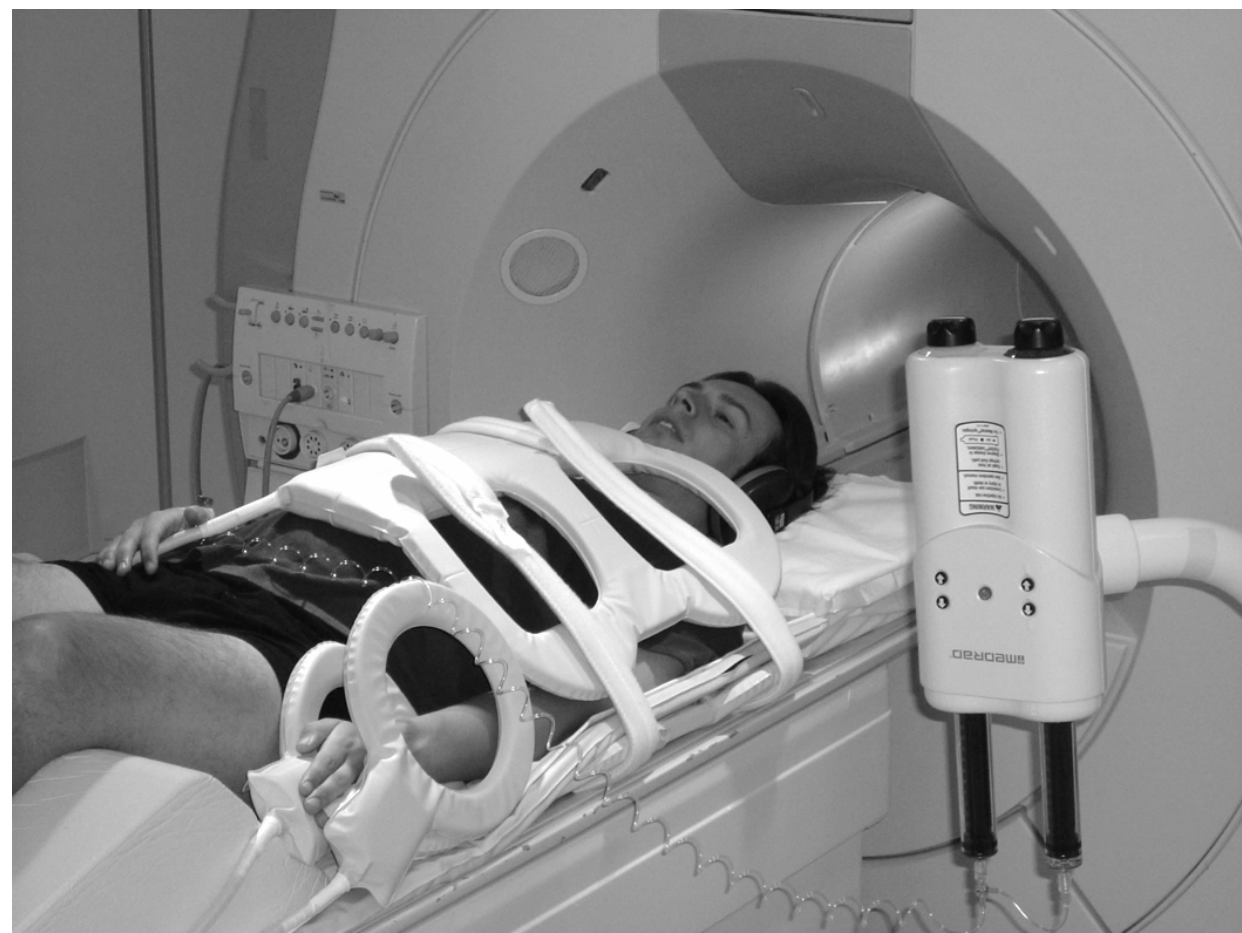

Figure 1: Patient and coil positioning for the CE-MRA examination. Note slight displacement of distal coil elements in relation to each other.

$\begin{array}{lll} & & \\ \text { Repetition time (msec) } & \text { Distal station } & \text { Proximal station } \\ \text { Echo time (msec) } & 5.5 & 5.1 \\ \text { Flip angle (degrees) } & 1.67 & 1.52 \\ \text { Field of view (mm) } & 40 & 40 \\ \text { Rectangular field of view (\%) } & 430 & 430 \\ \text { Matrix (scan / reconstruction) } & 35 & 85 \\ \text { Number of partitions } & 432 / 512 & 432 / 512 \\ \text { Slice thickness (mm) } & 100 & 80 \\ \text { SENSE factor } & 2.00 & 2.50 \\ \text { Acquired voxel size (mm }{ }^{3} \text { ) } & 2 & 2 \\ \text { Reconstructed voxel size (mm } \text { (m) }^{3} & 1.00 \times 1.43 \times 2.00 & 1.00 \times 1.42 \times 2.50 \\ \text { Scan duration (seconds) } & 0.84 \times 0.84 \times 1.00 & 0.84 \times 0.84 \times 1.30 \\ \text { Number of phases acquired } & 14.9 & 28.9 \\ & 3 & 2 \\ & & \end{array}$

Table 1: CE-MRA imaging parameters. 


\section{Distal arterial station \\ Proximal arterial station \\ Proximal venous station \\ Distal venous station}

\section{Contrast Medium}

19mL@3mL / sec

19mL@3mL/sec

40mL*@2mL/sec

60mL*@2mL/sec

\section{Saline Flush}

25mL@3mL / sec

25mL@3mL/sec

not given

not given

Table 2: CE-MRA injection protocols for imaging the upper extremity arteries and veins

In all patients, a total of $45 \mathrm{~mL}$ of gadopentetate dimeglumine (Gadolinium-DTPA, Magnevist, Schering, Berlin) was used to enhance intravascular signal, corresponding to between a double and triple dose. The use of up to a triple dose gadopentetate dimeglumine is widely accepted for imaging peripheral arterial occlusive disease (24-26). Furthermore, the use of gadopentetate dimeglumine in ESRD-patients is not contraindicated with regards to impairment of residual renal function (27). For venous imaging 1:15 diluted contrast medium was used $(6.7 \mathrm{~mL}$ Gd-DTPA mixed with $93.3 \mathrm{~mL}$ saline). A MR-compatible remote-controlled power injector (Medrad Spectris; Medrad, Indiana, PA) was used for contrast injection. The injection protocol is listed in table 2.

Appropriate synchronization of the start of the CE-MRA acquisition in relation to contrast medium arrival was achieved by using real-time bolus monitoring software (BolusTrak; Philips Medical Systems). For distal arterial imaging the acquisition was started three to four seconds after enhancement was first seen halfway the forearm. Proximal arterial imaging was started after enhancement of the distal brachial artery as determined from the distal arterial acquisition. Bolus monitoring was also applied for timing the proximal venous acquisition. Image acquisition was started after enhancement of the brachiocephalic vein was seen. Prior to imaging the forearm veins, an inflatable pressure cuff was applied around the upper arm (inflated to $60 \mathrm{mmHg}$ ). The 3D CE-MRA scan was initiated 5 seconds after the start of contrast medium injection. Total examination time for CE-MRA was approximately 35-45 minutes including patient positioning and post-processing. 


\section{Chapter 5}

\section{MR Image analysis}

After acquisition was completed the CE-MRA datasets were transferred to an off-line Windows XP workstation (Microsoft, Redmond, WA) with dedicated postprocessing software (Viewforum R4.2, Philips Medical Systems).

CE-MRA images were read in random order, in a prospective manner by a radiologist with 9 years experience in vascular imaging. The reviewer was informed about the exact level of DUS diameter measurements but was blinded for the results. Review was done on the 3D CE-MRA scans, which exhibited the best selective arterial and venous vessel enhancement. The reviewer used maximum intensity projections (MIPs) and multi-planar reformations (MPRs) and had the availability over all source images.

First, image quality was subjectively assessed on a 5 point scale (0: not visible, 1: visible but suboptimal depiction due to incomplete filling, blurring or other artifacts, 2: visible and interpretable, 3: visible and interpretable with good image quality, to 4: visible and interpretable with excellent image quality). A separate score was given for the image quality in the upper and lower station for both arterial and venous acquisitions, respectively.

After subjective image quality was determined the reviewer assessed arterial and venous diameter at the location that was chosen for surgery. Vascular diameters were assessed on cross-sectional reformations perpendicular to the center line of the vessel, using an electronic caliper with an accuracy of $0.1 \mathrm{~mm}$. Because vessel cross sections often exhibit an ellipsoid shape, the diameter was calculated by computing the numerical average of the length of the shortest and longest orthogonal axes in the ellipse as determined with the full width half maximum (FWHM) algorithm $(28,29)$.

\section{Duplex ultrasonography}

All DUS examinations were done by a licensed vascular technician with over 10 years experience in preoperative upper extremity vessel assessment. A commercially available duplex ultrasound scanner and $7.5 \mathrm{MHz}$ linear probe (Aloka 5500, Tokyo, Japan) were used for all preoperative DUS assessments. Ultrasound translucent gel was applied between skin and probe to avoid compression of veins. All examinations were performed according to Katz's vein 
mapping protocol in a warm room after 15 minutes of acclimatization (30). Diameters of the brachial -, radial - and ulnar arteries and accompanying veins, as well as the basilic and cephalic veins, were measured at predetermined locations in the distal forearm ( $5 \mathrm{~cm}$ proximal to wrist joint), halfway along the forearm, in the proximal forearm ( $5 \mathrm{~cm}$ distal to the antecubital crease), at the elbow joint, in the distal upper arm ( $5 \mathrm{~cm}$ proximal to the antecubital crease), halfway the upper arm, and in the proximal upper arm ( $5 \mathrm{~cm}$ distal to the axilla). Analogous to the 3D CE-MRA protocol, a tourniquet was applied around the upper arm to induce venous dilatation in the forearm prior to the measurement of forearm venous diameters (30).

During DUS acquisition arterial and venous diameters were measured in longitudinal and transverse orientation on the DUS machine using an electronic caliper with an accuracy of $0.1 \mathrm{~mm}$. Total examination time for DUS was approximately 60 minutes per patient.

Since DUS is the routinely used imaging modality for clinical purposes, the results of the DUS exam determined the type of AVF to be created as well as the exact location.

\section{Intra-operative measurements}

All patients were operated under regional anesthesia by axillary nerve blockage. During operation the artery and vein used to create the AVF were carefully handled to avoid vasospasm. The occurrence of vessel spasm was noted and papaverine in saline solution was available to relieve this whenever necessary. The internal diameter of both artery and vein were measured using coronary probes with diameters between 0.5-5.0 mm (in increments of $0.25 \mathrm{~mm}$ ). To determine the vessel diameter the probe was gently inserted into the lumen of the transected vessel. The reference diameter of the vessel was taken as the largest probe size that did not induce noticeable vessel contour deformation. The site of diameter measurement was determined by the preoperative DUS results. The intra-operative diameter measurements $(\mathrm{IO})$ using coronary probes served as the standard of reference. 


\section{Statistical analysis}

For both arteries and veins, the mean subjective image quality score was calculated for the CE-MRA images of all patients. Arterial and venous diameter measurements (CE-MRA and DUS) were compared to 10 measurements using the non-parametric Wilcoxon signed rank test (SPSS, version 10.0.5; SPSS Inc, Chicago, IL). $P$ values $<0.05$ were considered significant. Diameter measurement accuracy (mean difference between DUS and IO or CE-MRA and IO) as well as precision (standard deviation of the difference between DUS and IO or CE-MRA and IO) were calculated using the SPSS software. Differences in diameter measurement between DUS and $1 O$ and between CE-MRA and IO were plotted against the average (DUS $+1 \mathrm{IO} 2$ and CE-MRA $+1 \mathrm{I} / 2$ ) by means of BlandAltman plots $(31,32)$.

\section{Results}

All CE-MRA and DUS examinations and hemodialysis access creations (forearm radial-cephalic $A V F n=5$, upper arm brachial-cephalic $A V F n=3$, upper arm brachial-basilic AVF $n=11$, forearm polytetrafluoroethylene (PTFE) loop AVG $n$ $=6$ ) were performed successfully without any complication. During access creation and intra-operative diameter measurements no severe vessel spasm occurred. Upper extremity arteries and veins were depicted successfully with both CE-MRA and DUS in all cases. In figures 2 and 3, a typical example of CEMRA and corresponding DUS examination is shown. In figure 4 an example is shown of the two dynamic phases that were obtained in the proximal station.

CE-MRA yielded images with good to excellent subjective image quality for both arteries (upper station: $3.6 \pm 0.4$, lower station $3.4 \pm 0.6$ ) and veins (upper station $3.3 \pm 0.6$, lower station $3.5 \pm 0.5$ ).

Both CE-MRA and DUS accurately determined true forearm arterial diameters. Upper arm arterial diameters, in contrast were significantly overestimated compared to 10 measurements. DUS significantly underestimated venous diameters of both the forearm and upper arm, compared to $I O$ measurements. CE-MRA accurately assessed venous diameter compared to 10 measurements (table 3). 
Differences between CE-MRA and DUS diameter measurements compared to IO measurements were visualized in Bland-Altman plots (figure 5). The accuracy and precision for arterial diameter measurement were: $0.4 \mathrm{~mm}$ and $\pm 0.8 \mathrm{~mm}$ for CEMRA, and $0.6 \mathrm{~mm}$ and $\pm 1.2 \mathrm{~mm}$ for DUS, respectively. For venous diameter measurements by CE-MRA and DUS the accuracy and precision were: $0.3 \mathrm{~mm}$ and $\pm 0.7 \mathrm{~mm}$ and $1.2 \mathrm{~mm}$ and $\pm 1.2 \mathrm{~mm}$, respectively.

\begin{tabular}{|c|c|c|c|c|c|}
\hline \multirow[t]{2}{*}{ Arterial } & \multicolumn{3}{|c|}{ Mean Vessel Diameter $(\mathrm{mm} \pm S D)$} & \multicolumn{2}{|l|}{$P$-value } \\
\hline & 10 & CE-MRA & DUS & 10 vs CE-MRA & IOVsDUS \\
\hline Distal $(n=5)$ & $3.00( \pm 0.35)$ & $2.94( \pm 0.67)$ & $2.80( \pm 0.48)$ & 0.89 & 0.59 \\
\hline Proximal $(n=20)$ & $3.55( \pm 0.51)$ & $4.05( \pm 0.84)$ & $4.38( \pm 1.24)$ & $0.02 *$ & $0.00^{*}$ \\
\hline \multirow[t]{2}{*}{ Venous } & \multicolumn{3}{|c|}{ Mean Vessel Diameter $(m m \pm S D)$} & \multicolumn{2}{|l|}{$P$-value } \\
\hline & 10 & $C E-M R A$ & DUS & 10 vs CE-MRA & IOvsDUS \\
\hline Distal $(n=5)$ & $3.40( \pm 0.22)$ & $2.64( \pm 0.61)$ & $2.50( \pm 0.44)$ & 0.08 & $0.04^{\star}$ \\
\hline Proximal $(n=20)$ & $4.30( \pm 0.78)$ & $4.09( \pm 0.71)$ & $3.02( \pm 1.65)$ & 0.17 & $0.00^{*}$ \\
\hline
\end{tabular}

Table 3: Diameter measurement of upper extremity arteries and veins by CE-MRA, DUS and IO. Values represent means ( \pm standard deviation), ${ }^{\star}=\mathrm{P}<0.05$. 


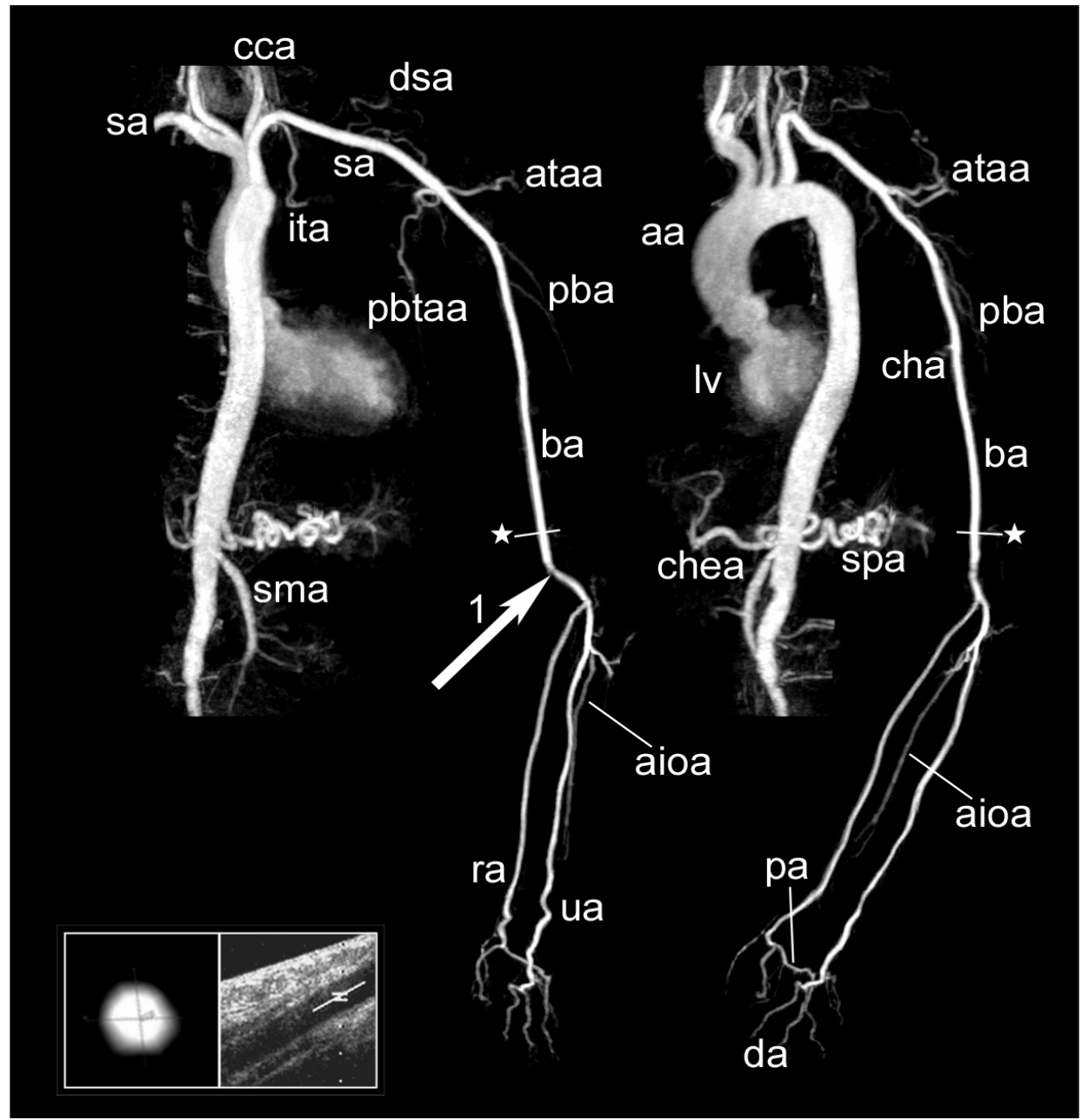

Figure 2: Coronal (left) and left anterior oblique (right) whole volume maximum intensity projections of the 3D CE-MRA arterial acquisitions obtained in a patient with a previous brachio-cephalic arteriovenous loop graft at the elbow in the left arm. The arrow (1) points at a stenosis located proximal to the brachial artery bifurcation at the level of the former arteriovenous anastomosis. The planned anastomosis of the new native brachio-cephalic arteriovenous fistula and level of diameter measurements is pointed out by the bar with the star. The inset in the lower left corner is the crosssectional depiction of the brachial artery at the level of the bar with the star. This cross sectional depiction, perpendicular to the vessel course, was used for diameter measurements using the FWHM algorithm. The corresponding B-mode ultrasound depiction in a longitudinal fashion is shown on the right. Legend: Iv = left ventricle, aa = aortic arch, sa = subclavian artery, cca = left common carotid artery, ita = internal thoracic artery, dsa = dorsal scapular artery, ataa = acromial branch of the thoracoacromial artery, pbtaa $=$ posterior branch of the thoracoacromial artery, pba $=$ profunda brachial artery, ba = brachial artery, aioa = anterior interosseous artery, ra $=$ radial artery, ua $=$ ulnar artery, $\mathrm{pa}=$ palmar arch, da = digital arteries, cha $=$ common hepatic artery, $\mathrm{spa}=$ splenic artery, sma $=$ superior mesenteric artery. 


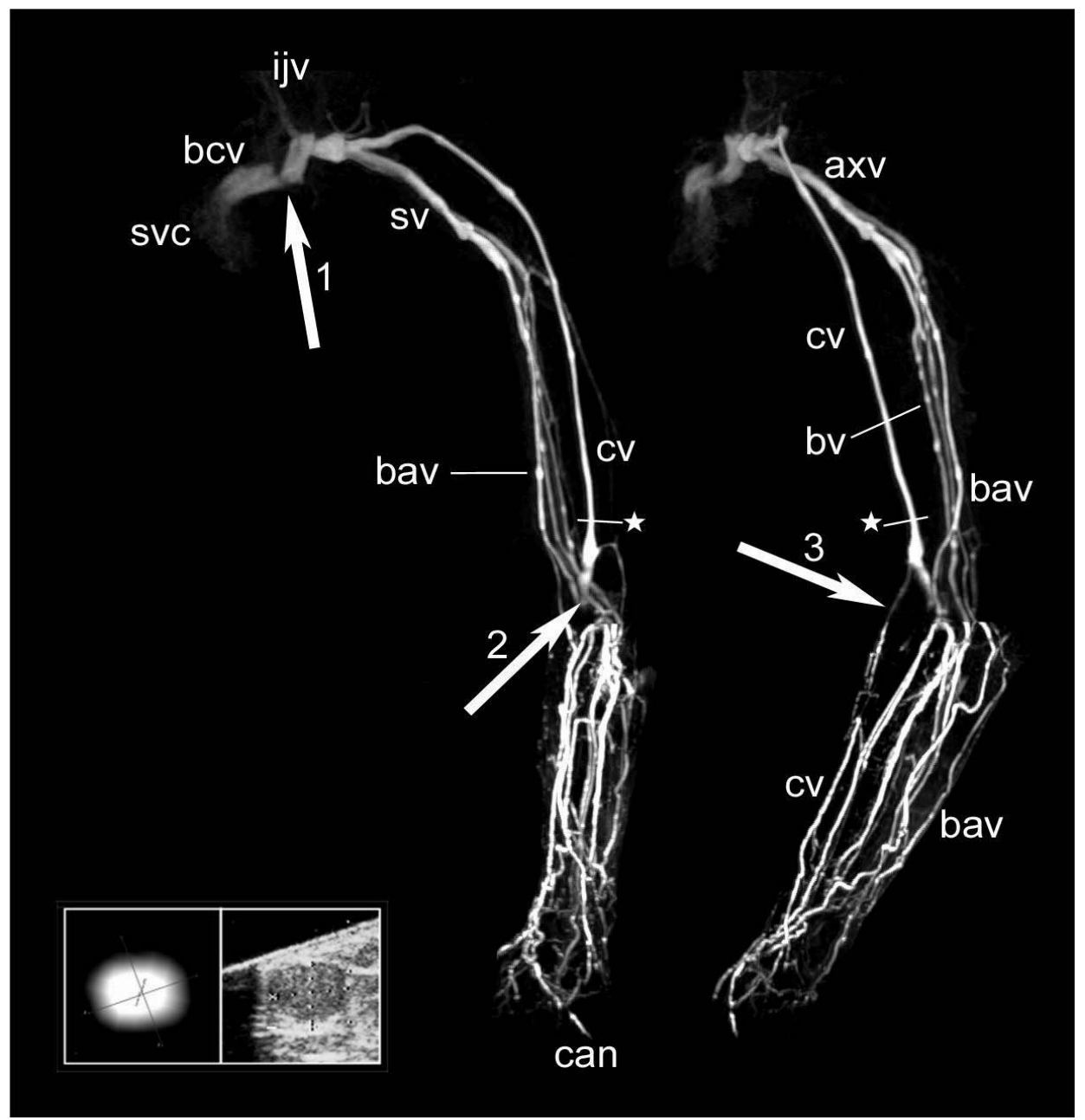

Figure 3: Coronal (left) and left anterior oblique maximum intensity projections of the 3D CE-MRA venous acquisitions in the same patient. Arrow 1 points at pseudostenosis caused by posterior compression of the left brachiocephalic vein by the brachiocephalic artery. The second arrow points at the site of the former venous anastomosis of the thrombosed arteriovenous loop graft. The third arrow points at the cephalic vein distal to the elbow joint which is patent but fragile. The inset in the lower left corner shows the cross-sectional depiction of the cephalic vein at the level of the bar with the star in the left panel. This cross sectional depiction, perpendicular to the vessel course, was used for diameter measurements using the full-width half maximum (FWHM) algorithm. In the right panel the corresponding B-mode ultrasonography image is shown. Legend: svc = superior vena cava, bcv = brachio-cephalic vein, ijv = internal jugular vein, sv = subclavian vein, axv = axillary vein, $\mathrm{cv}=$ cephalic vein, bv = brachial vein, bav = basilic vein, can = intravenous cannula for contrast media injection. 


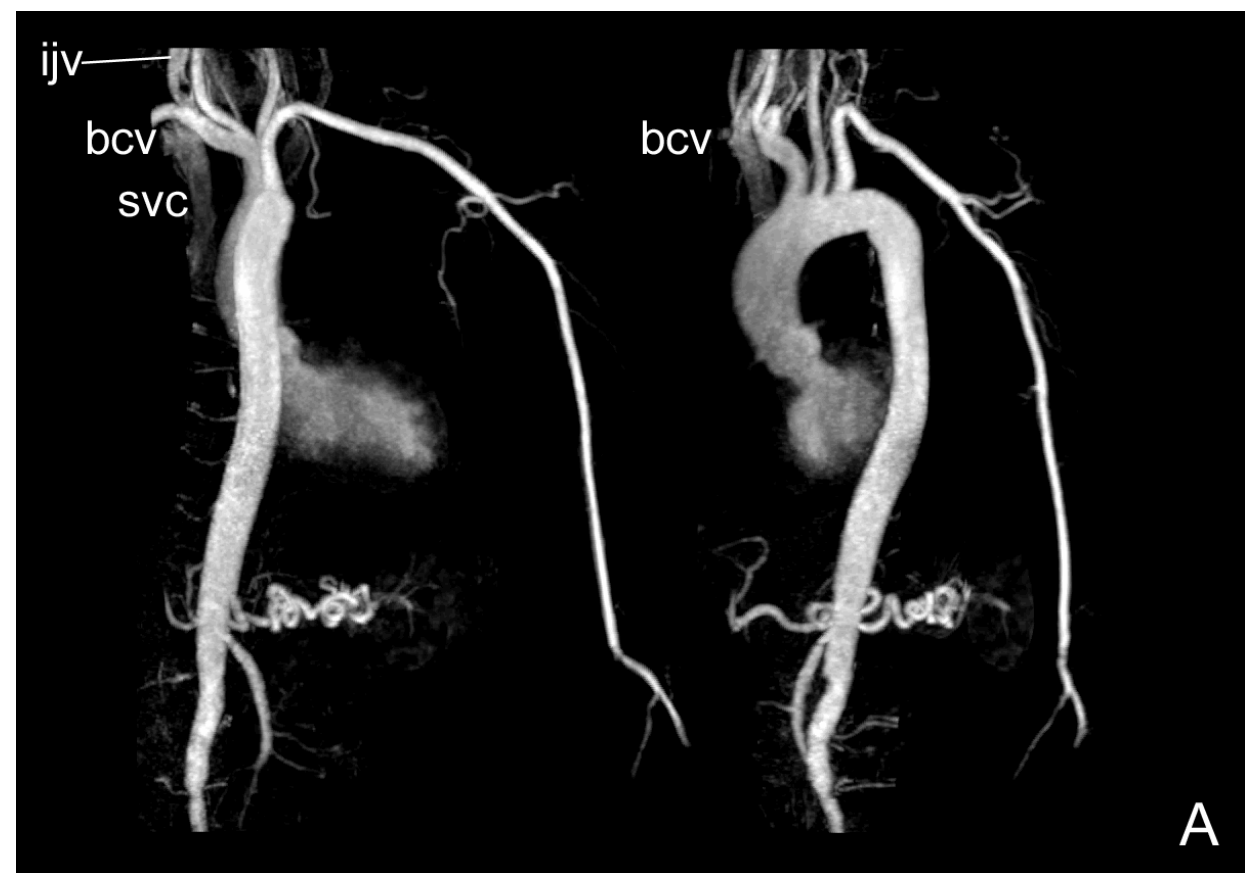

Figure 4A: Coronal (left) and left anterior oblique (right) maximum intensity projections of the first of two dynamic scans of the 3D CE-MRA arterial acquisitions from the same patient as shown in figure 2. The relatively short arteriovenous transit time of contrast medium in the cephalic circulation enables depiction of the jugular, subclavian and brachiocephalic veins and part of the superior vena cava during the first dynamic scan. Legend: svc = superior vena cava, bcv = brachiocephalic vein, ijv $=$ internal jugular vein. (B) Coronal (left) and left anterior oblique (right) maximum intensity projections of the second of two dynamic scans of the 3D CE-MRA arterial acquisitions from the same patient as shown in figure 2. Although arterial vessel-to-background contrast is suboptimal, the second phase is well suited to assess patency of the jugular, subclavian and brachiocephalic veins as well as the superior vena cava. Legend: svc = superior vena cava, bcv = brachio-cephalic vein, ijv = internal jugular vein, $\mathrm{sv}=$ subclavian vein, $\mathrm{axv}=$ axillary vein, $\mathrm{cv}=$ cephalic vein, $\mathrm{bv}=$ basilica vein, $\mathrm{av}=$ azygos vein, $\mathrm{pv}=$ portal vein, $\mathrm{spv}=$ splenic vein, $\mathrm{smv}=$ superior mesenteric vein, icv $=$ inferior vena cava. 


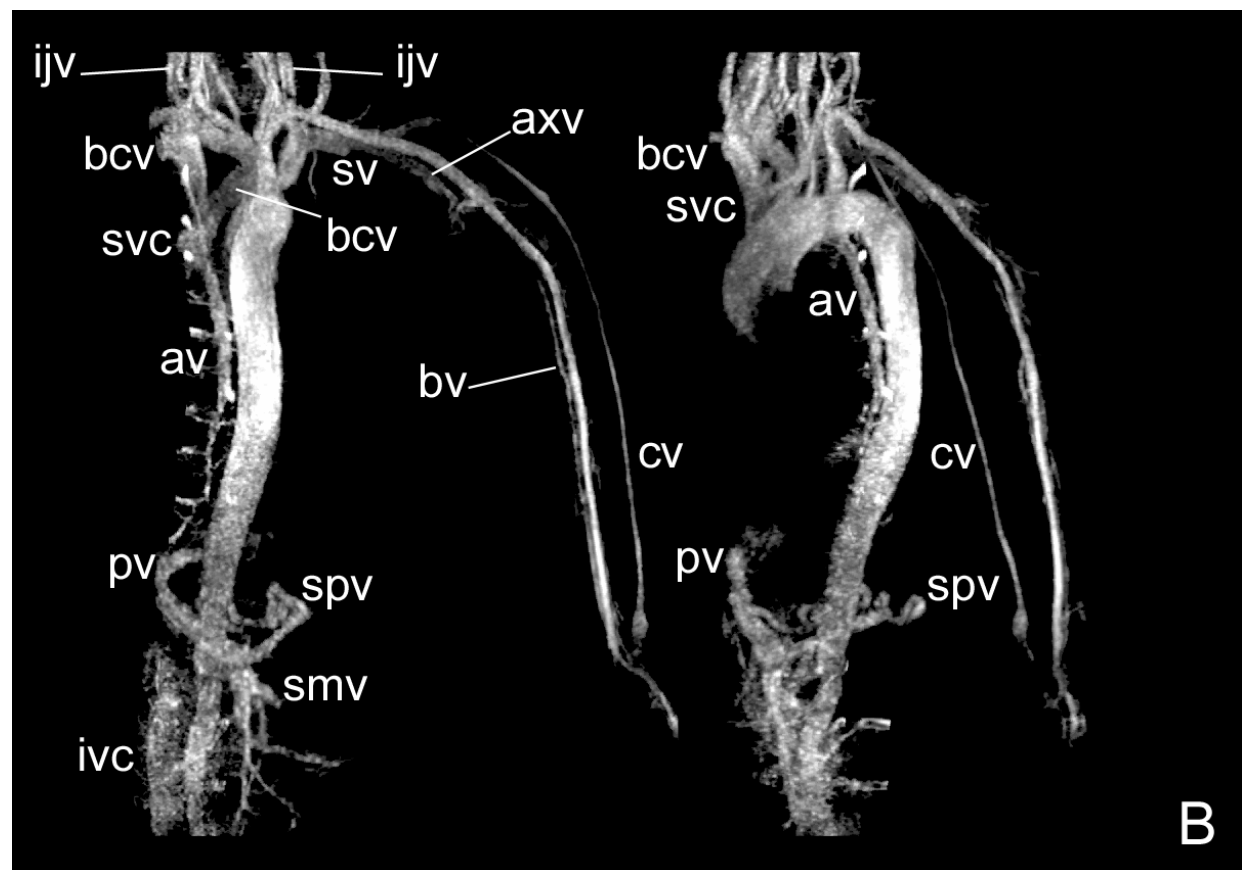

Figure 4B: Coronal (left) and left anterior oblique (right) maximum intensity projections of the second of two dynamic scans of the 3D CE-MRA arterial acquisitions from the same patient as shown in figure 2. Although arterial vessel-to-background contrast is suboptimal, the second phase is well suited to assess patency of the jugular, subclavian and brachiocephalic veins as well as the superior vena cava. Legend: svc = superior vena cava, bcv = brachio-cephalic vein, ijv = internal jugular vein, $\mathrm{sv}=$ subclavian vein, axv = axillary vein, $\mathrm{cv}=$ cephalic vein, bv = basilica vein, av = azygos vein, $\mathrm{pv}$ $=$ portal vein, $\mathrm{spv}=$ splenic vein, $\mathrm{smv}=$ superior mesenteric vein, icv $=$ inferior vena cava. 

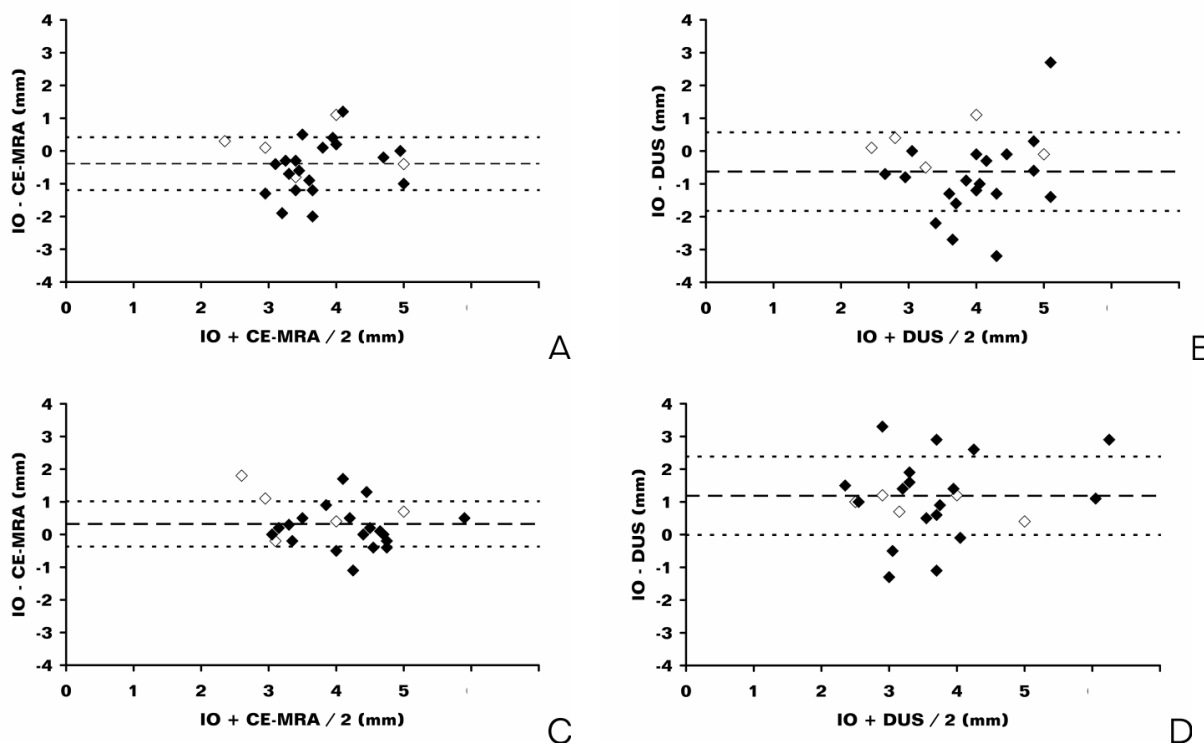

Figure 5: Bland-Altman plots of mean diameter (x-axis) measurements versus the difference in diameter measurements (y-axis). Upper left (A): mean IO and CE-MRA arterial diameter measurements versus the difference of measurements. Upper right (B), mean IO and DUS arterial diameter measurements versus the difference of measurements. Lower left (C): mean IO and CEMRA venous diameter measurements versus the difference of measurements. Lower right (D), mean IO and DUS venous diameter measurements versus the difference of measurements. The solid line represents the mean difference and the dotted lines the standard deviation. The black and white diamonds represent upper arm and forearm arterial diameter measurements, respectively.

\section{Discussion}

In the present study we present a simple CE-MRA protocol that was feasible and enabled selective depiction of upper extremity arteries and veins with high image quality in a single imaging session in all patients. CE-MRA and DUS assessment of forearm arterial diameters corresponded reasonably well with 10 measurements although the sample size was small. Both CE-MRA and DUS overestimated arterial diameters in the upper arm compared to true arterial diameter. For determination of venous diameter, however, measurements based on CE-MRA images were more accurate than diameter measurements based on DUS (when IO measurements were used as the standard of reference).

The importance of accurate assessment of vascular diameters in the upper extremity is reflected in the recommendation by both the National Kidney 
Foundation Dialysis Outcome Quality Initiative (NKF-DOQI) and European Vascular Access Society Guidelines to perform extensive preoperative diagnostics with the aim to reduce early failure and non-maturation of newly created AVFs $(4,5)$. Both physical examination and duplex assessment of upper extremity arteries and veins are the diagnostic modalities recommended for routine clinical use by these guidelines $(4,5)$. Arterial and venous diameters are the parameters that have been studied most extensively as predictors of surgical outcome in terms of AVF and AVG function. However, the predictive value of preoperative measurements such as diameters, flows and flow-velocities remain subject of discussion $(10,18,22)$. Suggested arterial and venous cut-off diameters for prediction of successful AVF outcome range from 1.6 to $2.5 \mathrm{~mm}$ for forearm AVFs (6-16). In light of the current findings one might speculate that this inconsistency may be due to variations in vascular diameter due to the use of different diagnostic modalities and measurement techniques.

The current study was motivated by the fact that CE-MRA is capable of imaging vascular structures over a large field of view in truly 3D fashion with high spatial resolution in short imaging times. These capabilities have resulted in highly accurate depiction and detection of disease in cervical, thoracic, abdominal and peripheral arteries as well as veins $(26,33-45)$. Other investigators have used various MRA techniques to depict the upper extremity vasculature (36, 37, 39, 42-50). However, in none of the previous reports did the authors assess both arteries and veins in a single exam. In part this is due to the fact that non contrast-enhanced flow dependent techniques such as time-of-flight and phasecontrast MRA are not suitable for imaging upper extremity vasculature because of well-known artifacts, and even more important, because of the inability to depict veins in the absence of flow, as is the case during cuff congestion $(40,51)$. To our knowledge, this is the first study combining arterial as well as venous imaging in a single imaging session. With regard to the CE-MRA imaging protocol it is important to stress that the order in which the acquisitions should be performed is crucial to obtain optimal arterial image quality without unwanted venous enhancement. Furthermore, careful timing of arterial acquisition is important to achieve the optimal vessel to background enhancement (34). The CE-MRA protocol as described in this study enables selective depiction of both arteries and veins of the entire upper extremity, including the intra-thoracic parts 
of these vessels, with good to excellent image quality. The imaging technique did not result in unwanted enhancement of either veins or arteries during arterial and venous acquisitions, respectively.

Comparison of diameter measurements by CE-MRA, DUS and IO yielded interesting discrepancies. Upper arm arterial diameters were overestimated significantly by both CE-MRA and DUS whereas forearm arterial diameters were determined accurately by both CE-MRA and DUS. CE-MRA was found to be accurate for determination of both forearm and upper arm venous diameters. DUS, on the other hand, underestimated both forearm and upper arm venous diameters. In contrast to our findings Malovrh et al. reported a good correlation between 10 measurements and preoperative DUS diameter measurements of forearm superficial veins without application of venous congestion (DUS: $2.3 \pm 0.7$ $\mathrm{mm}$; IO: $2.4 \mathrm{~mm}$ [no standard deviation given]) (7). Subsequent application of venous congestion ( $50 \mathrm{mmHg}$ ) resulted in a $45 \%$ increase of the luminal diameter to $3.3 \pm 1.2 \mathrm{~mm}$, leading to overestimation when compared to the 10 findings (7). A possible explanation for this discrepancy might be that calipers instead of coronary probes were used to determine vascular diameters intra-operatively (7). Also, it was not stated how these authors dealt with the ellipsoid shape of the veins at DUS. Alternatively, although intra-operative findings in general are being considered as a good standard of reference, this might not hold for vessel diameter measurements. Furthermore, the accuracy of 10 measurements was limited to $0.25 \mathrm{~mm}$ because the probes used had size increments of $0.25 \mathrm{~mm}$. To the best of our knowledge there are no data available on the accuracy and reproducibility of intra-operative diameter measurements. However, the method we used is generally accepted in the field of coronary artery bypass grafting to determine vessel diameters and patency and the variations we encountered exceed probe size variation.

The current study has several additional limitations. Despite attempts at careful co-localization, we cannot exclude slight differences in location of CE-MRA, DUS and $1 \mathrm{O}$ diameter measurements. In addition, because the diameter measurements with different modalities were performed on different days, it is likely that physiological variations in vessel diameter are partially responsible for the discrepancies. This is substantiated by a recent study by Planken et al. who reported substantial day-to-day variations in vascular diameters (52). Further 
explanations for the differences between modalities may be the altered vascular tone and subsequent increase in venous diameter after axillary nerve block as well as effect of surgical vessel manipulations. A decrease in vascular tone due to anesthetic drugs, however, should have led to larger IO vessel calibers and hence underestimation of both arterial and venous diameters with both CE-MRA and DUS. A methodological difference between CE-MRA and DUS measurements was the use of an inflatable pressure cuff at CE-MRA versus a tourniquet at DUS measurements. However, a recent study found that maximum venous diameter measured after application of an inflatable cuff does not differ significantly when compared to the use of a tourniquet (52). Finally, this study was carried out in a relatively small group of patients and further study is needed to determine if CEMRA of the upper extremity can be used in routine clinical practice.

In conclusion, the presented CE-MRA protocol is feasible and enables selective imaging of upper extremity vasculature in patients requiring a vascular access for hemodialysis. Forearm arterial diameters can be assessed accurately by CEMRA. Both CE-MRA and DUS slightly underestimate upper arm arterial diameters. In comparison to DUS, CE-MRA enables a more accurate determination of upper extremity venous diameters. Future prospective studies are needed to determine if the use of CE-MRA will ultimately lead to reduced early failure and non maturation rates in patients undergoing access surgery. 


\section{References}

1. Grassmann A, Gioberge S, Moeller S, Brown G. ESRD patients in 2004: global overview of patient numbers, treatment modalities and associated trends. Nephrol Dial Transplant 2005; 20:2587-2593.

2. Meichelboeck W. ESRD 2005 - A Worldwide Overview - Facts, Figures and Trends. In: Mickley V, ed. 4th International Congress of the Vascular Access Society (VAS). Berlin, Germany: Blood Purification, Karger, 2005; 227-261.

3. Robbin ML, Chamberlain NE, Lockhart ME, et al. Hemodialysis arteriovenous fistula maturity: US evaluation. Radiology 2002; 225:59-64.

4. III. NKF-K/DOQI Clinical Practice Guidelines for Vascular Access: update 2000. Am J Kidney Dis $2001 ; 37:$ S137-181.

5. Tordoir JH, Mickley V. European guidelines for vascular access: clinical algorithms on vascular access for haemodialysis. Edtna Erca J 2003; 29:131-136.

6. Robbin ML, Gallichio MH, Deierhoi MH, Young CJ, Weber TM, Allon M. US vascular mapping before hemodialysis access placement. Radiology 2000; 217:83-88.

7. Malovrh M. Native arteriovenous fistula: preoperative evaluation. Am J Kidney Dis 2002; 39:1218-1225.

8. Malovrh M. Non-invasive evaluation of vessels by duplex sonography prior to construction of arteriovenous fistulas for haemodialysis. Nephrol Dial Transplant 1998; 13:125-129.

9. Silva MB, Jr., Hobson RW, 2nd, Pappas PJ, et al. A strategy for increasing use of autogenous hemodialysis access procedures: impact of preoperative noninvasive evaluation. J Vasc Surg 1998; 27:302-307; discussion 307-308.

10. Lemson MS, Leunissen KM, Tordoir JH. Does pre-operative duplex examination improve patency rates of Brescia-Cimino fistulas? Nephrol Dial Transplant 1998; 13:1360-1361.

11. Leblanc M, Saint-Sauveur E, Pichette V. Native arterio-venous fistula for hemodialysis: What to expect early after creation? The Journal of Vascular Access 2003; 4:39-44.

12. Mendes RR, Farber MA, Marston WA, Dinwiddie LC, Keagy BA, Burnham SJ. Prediction of wrist arteriovenous fistula maturation with preoperative vein mapping with ultrasonography. $J$ Vasc Surg 2002; 36:460-463.

13. Tordoir JH, Rooyens P, Dammers R, van der Sande FM, de Haan M, Yo TI. Prospective evaluation of failure modes in autogenous radiocephalic wrist access for haemodialysis. Nephrol Dial Transplant 2003; 18:378-383.

14. Wong V, Ward R, Taylor J, Selvakumar S, How TV, Bakran A. Factors associated with early failure of arteriovenous fistulae for haemodialysis access. Eur J Vasc Endovasc Surg 1996; 12:207-213.

15. Brimble KS, Rabbat Ch G, Treleaven DJ, Ingram AJ. Utility of ultrasonographic venous assessment prior to forearm arteriovenous fistula creation. Clin Nephrol 2002; 58:122-127. 
16. Ascher $E$, Gade $P$, Hingorani $A$, et al. Changes in the practice of angioaccess surgery: impact of dialysis outcome and quality initiative recommendations. J Vasc Surg 2000; 31:84-92.

17. Patel ST, Hughes J, Mills JL, Sr. Failure of arteriovenous fistula maturation: an unintended consequence of exceeding dialysis outcome quality initiative guidelines for hemodialysis access. J Vasc Surg 2003; 38:439-445; discussion 445.

18. Lockhart ME, Robbin ML, Allon M. Preoperative sonographic radial artery evaluation and correlation with subsequent radiocephalic fistula outcome. J Ultrasound Med 2004; 23:161-168; quiz 169-171.

19. Huber TS, Ozaki CK, Flynn TC, et al. Prospective validation of an algorithm to maximize native arteriovenous fistulae for chronic hemodialysis access. J Vasc Surg 2002; 36:452-459.

20. Miller PE, Tolwani A, Luscy CP, et al. Predictors of adequacy of arteriovenous fistulas in hemodialysis patients. Kidney Int 1999; 56:275-280.

21. Mihmanli I, Besirli K, Kurugoglu S, et al. Cephalic vein and hemodialysis fistula: surgeon's observation versus color Doppler ultrasonographic findings. J Ultrasound Med 2001; 20:217222.

22. Allon M, Robbin ML. Increasing arteriovenous fistulas in hemodialysis patients: problems and solutions. Kidney Int 2002; 62:1109-1124.

23. Lemson MS, Zwiers I, Leunissen KML, Tordoir JHM. Patient Selection with Preoperative Duplex Examination Can Improve Patnecy Rates of Brescia-Cimino Fistulas. The Journal Of Vascular Technology 1999; 23:115-119.

24. Meaney JF. Magnetic resonance angiography of the peripheral arteries: current status. Eur Radiol 2003; 13:836-852.

25. Leiner T. Magnetic resonance angiography of abdominal and lower extremity vasculature. Top Magn Reson Imaging 2005; 16:21-66.

26. Yucel EK, Anderson CM, Edelman RR, et al. AHA scientific statement. Magnetic resonance angiography : update on applications for extracranial arteries. Circulation 1999; 100:2284-2301.

27. Geoffroy O, Tassart M, Le Blanche AF, et al. Upper extremity digital subtraction venography with gadoterate meglumine before fistula creation for hemodialysis. Kidney Int 2001; 59:14911497.

28. Hoogeveen RM, Bakker CJ, Viergever MA. Limits to the accuracy of vessel diameter measurement in MR angiography. J Magn Reson Imaging 1998; 8:1228-1235.

29. Westenberg JJ, van der Geest RJ, Wasser MN, et al. Vessel diameter measurements in gadolinium contrast-enhanced three-dimensional MRA of peripheral arteries. Magn Reson Imaging 2000; 18:13-22.

30. Katz ML, Comerota AJ, DeRojas J, Bowman G, Czeredarczuk M, White JV. B-mode imaging to determine the suitability of arm veins for primary arteriovenous fistulae. The Journal of Vascular Technology 1987; 11:172-174.

31. Bland JM, Altman DG. Statistical methods for assessing agreement between two methods of clinical measurement. Lancet 1986; 1:307-310. 


\section{Chapter 5}

32. Bland JM. An introduction to medical statistics. Oxford: Oxford University Press, 1987.

33. Willinek WA, von Falkenhausen M, Born M, et al. Noninvasive detection of steno-occlusive disease of the supra-aortic arteries with three-dimensional contrast-enhanced magnetic resonance angiography: a prospective, intra-individual comparative analysis with digital subtraction angiography. Stroke 2005; 36:38-43.

34. Willinek WA, Gieseke J, Conrad R, et al. Randomly segmented central k-space ordering in highspatial-resolution contrast-enhanced MR angiography of the supraaortic arteries: initial experience. Radiology 2002; 225:583-588.

35. Ruehm SG, Wiesner W, Debatin JF. Pelvic and lower extremity veins: contrast-enhanced threedimensional MR venography with a dedicated vascular coil-initial experience. Radiology 2000; 215:421-427.

36. Ruehm SG, Zimny K, Debatin JF. Direct contrast-enhanced 3D MR venography. Eur Radiol $2001 ; 11: 102-112$.

37. Ruehm SG. MR venography. Eur Radiol 2003; 13:229-230.

38. Quinn SF, Sheley RC, Semonsen KG, Leonardo VJ, Kojima K, Szumowski J. Aortic and lowerextremity arterial disease: evaluation with MR angiography versus conventional angiography. Radiology 1998; 206:693-701.

39. Ho VB, Prince MR, Dong Q. Magnetic resonance imaging of the aorta and branch vessels. Coron Artery Dis 1999; 10:141-149.

40. Ho KY, Leiner T, de Haan MW, van Engelshoven JM. Peripheral MR angiography. Eur Radiol 1999; 9:1765-1774.

41. Leiner T, Kessels AG, Nelemans PJ, et al. Peripheral arterial disease: comparison of color duplex US and contrast-enhanced MR angiography for diagnosis. Radiology 2005; 235:699-708.

42. Goyen M, Barkhausen J, Kuehl H, et al. [Contrast-enhanced 3D MR venography of central thoracic veins: preliminary experience]. Rofo Fortschr Geb Rontgenstr Neuen Bildgeb Verfahr 2001; 173:356-361.

43. Kroencke TJ, Taupitz M, Arnold R, Fritsche L, Hamm B. Three-dimensional gadolinium-enhanced magnetic resonance venography in suspected thrombo-occlusive disease of the central chest veins. Chest 2001; 120:1570-1576.

44. Shankar KR, Abernethy LJ, Das KS, et al. Magnetic resonance venography in assessing venous patency after multiple venous catheters. J Pediatr Surg 2002; 37:175-179.

45. Wentz KU, Frohlich JM, von Weymarn C, Patak MA, Jenelten R, Zollikofer CL. High-resolution magnetic resonance angiography of hands with timed arterial compression (tac-MRA). Lancet 2003; 361:49-50.

46. Haire WD, Lynch TG, Lund GB, Lieberman RP, Edney JA. Limitations of magnetic resonance imaging and ultrasound-directed (duplex) scanning in the diagnosis of subclavian vein thrombosis. J Vasc Surg 1991; 13:391-397.

47. Laissy JP, Fernandez P, Karila-Cohen P, et al. Upper limb vein anatomy before hemodialysis fistula creation: cross-sectional anatomy using MR venography. Eur Radiol 2003; 13:256-261. 
48. Polak JF, Fox LA. MR assessment of the extremity veins. Semin Ultrasound CT MR 1999; 20:36-46.

49. Schuman ES, F. QS, Standage BA, et al. Magnetic Resonce Angiography in evaluation of central and peripheral vasculature prior to dialysis surgery. Vascular access for hemodialysis-VI. Chicago, W.L. Gore \& Associates, and Precept Press 1999:103-109.

50. Shinde TS, Lee VS, Rofsky NM, Krinsky GA, Weinreb JC. Three-dimensional gadoliniumenhanced MR venographic evaluation of patency of central veins in the thorax: initial experience. Radiology 1999; 213:555-560.

51. Mihmanli I, Kantarci F. MR venography needs to know where it stands for vascular mapping prior to fistula creation. Eur Radiol 2003; 14:1130-1131.

52. Planken RN, Keuter XH, Hoeks AP, et al. Diameter measurements of the forearm cephalic vein prior to vascular access creation in end-stage renal disease patients: graduated pressure cuff versus tourniquet vessel dilatation. Nephrol Dial Transplant 2006; $21: 802-806$. 



\section{Chapter 6}

CE-MRA for prediction of hemodialysis vascular access early failure and nonmaturation:

a prospective multi-center study

R.N. Planken, T. Leiner, R.J. Nijenhuis, L.E.M. Duijm, P.W. Cuypers, P. Douwes-Draaijer, F.M. van der Sande, J.P. Kooman, M.W. de Haan, A.G. Kessels, J.H.M. Tordoir

Submitted 


\section{Abstract}

Background: Objective of the present study was to prospectively determine the value of contrast-enhanced magnetic resonance angiography (CE-MRA) for prediction of early failure and non-maturation of newly created hemodialysis vascular accesses.

Materials and methods: Seventy-three patients with end-stage renal disease underwent DUS and CE-MRA prior to vascular access creation. Assessment included detection of upper extremity vessel stenoses and occlusions. Two observers read the CE-MRA images for determination of inter-observer agreement and access surgical strategy. Access surgery was determined by duplex ultrasound (DUS) parameters. A vascular access was considered functional if it could be used for successful two-needle hemodialysis therapy within 2 months after creation.

Results: Six stenosed, 8 occluded arterial vessel segments and 12 stenosed and 41 occluded venous vessel segments were detected in 70 patients. Interobserver agreement for detection of upper extremity arterial and venous stenoses and occlusions with CE-MRA was substantial to almost perfect. DUS had a positive predictive value of $70 \%$. CE-MRA had a sensitivity, specificity, positive predictive value and negative predictive value of $98 \%, 33 \%, 77 \%$ and $88 \%$, respectively.

Conclusion: CE-MRA enables detection of upper extremity arterial and venous stenosis and occlusions, related with vascular access early failure and nonmaturation, with substantial to almost perfect inter-observer agreement. CEMRA is a valuable tool for prediction of vascular access early failure and nonmaturation with a better positive predictive value than DUS. 


\section{Introduction}

The estimated number of patients requiring dialysis treatment because of endstage renal disease (ESRD) has risen rapidly with about 20\% from 2001 (1.14 million) to 2005 (1.37 million) worldwide $(1,2)$. Despite many improvements in preoperative diagnostics as well as surgical technique, vascular access dysfunction after initial creation and the subsequent inability of adequate hemodialysis remains a serious problem.

Two distinct causes of access dysfunction, early failure and non-maturation, are generally identified. Early failure due to access thrombosis occurs within days to weeks after creation whereas non-maturation refers to a lack of vessel diameter and flow increase within weeks to months after creation. Both early failure and non-maturation result in a vascular access that is unsuitable for hemodialysis, leading to increased vascular access related patient morbidity, mortality and related costs (3-5). Prediction and ultimately prevention of vascular access early failure and non-maturation is therefore important.

Although the exact mechanisms of early failure and non-maturation remain elusive, it is recognized that preoperative assessment of upper extremity vessels can reduce early failure and non-maturation rates (3-5). Patients with preoperative small vessel diameters, low arterial inflow and venous outflow stenoses or occlusions have a higher risk of early access failure and nonmaturation. Because of its widespread availability and noninvasive character, duplex ultrasonography (DUS) is applied in the preoperative workup to assess vessel diameters and to detect arterial inflow or venous outflow stenosis and occlusions. Preoperative arterial and venous diameters of less than 1.6-2.5 mm, as determined by DUS, have been associated with a higher incidence of early failure and non-maturation of newly created vascular accesses (6-14). However, even after implementation of preoperative DUS assessment, reported early failure rates of autogenous arteriovenous fistulas (AVF) remain up to $50 \%(6,13$, $15,16)$. The persistently high early failure and non-maturation rates create the need for additional preoperative diagnostic tools to further reduce the incidence of hemodialysis vascular access early failure and non-maturation.

A promising imaging modality for the depiction of upper extremity arterial and venous anatomy is contrast-enhanced magnetic resonance angiography (CE- 
MRA) (17). This technique potentially allows for rapid arterial and venous mapping and in contrast to DUS, enables true three-dimensional imaging over a large field of view (17).

The objective of the present study was to prospectively determine the value of contrast-enhanced magnetic resonance angiography (CE-MRA) for prediction of early failure and non-maturation of newly created hemodialysis vascular accesses.

\section{Materials and Methods}

\section{Study design and population}

The study took place in a tertiary referral university hospital and a large nonacademic center, both with dedicated dialysis access services. All patients referred to the departments of surgery in both hospitals for creation of a new hemodialysis vascular access, were considered potential candidates for this prospective bi-center study. According to the National Kidney Foundation Kidney Disease Outcome Quality Initiative (NKF K-DOQI) guidelines and clinical practice guidelines for hemodialysis adequacy update 2006, patients with ESRD were referred to the surgical department for creation of a hemodialysis vascular access when creatinine clearance dropped below $25 \mathrm{~mL} / \mathrm{min}$ or if there was an anticipated need for hemodialysis within 1 year $(4,5)$. The medical ethics committees of both institutions approved the study and all patients signed informed consent prior to inclusion. Patients who did not meet the safety guidelines and recommendations for MR safety were excluded from this study as well as patients with claustrophobia, obesity and spastic paralysis.

\section{Standard preoperative assessment and selection of vascular access}

Physical examination of the entire upper extremity complemented by comprehensive DUS examination is the standard preoperative examination prior to vascular access creation in the 2 institutions. Physical examination was done by the vascular surgeons; DUS examination was performed by dedicated vascular technicians with over 10 years of experience. The ultrasound scanners used in this study were the Aloka 5500 (Aloka, Tokyo, Japan) and the Acuson Aspen (Acuson/Siemens, Malvern, PA, USA), both high-end systems dedicated for general ultrasound examinations and equipped with a $7.5 \mathrm{MHz}$ linear probe. 
Assessment included determination of forearm and upper arm arterial and venous patency, vessel diameter and characterization of of stenoses and obstructions. Superficial veins were assessed after venous congestion by a proximally applied tourniquet $(3,4,18,19)$. The following vessels were assessed (from proximal to distal): the subclavian, axillary, brachial, ulnar and radial arteries, and the subclavian, axillary, brachial, basilic and cephalic veins.

According to the NKF K-DOQI guidelines and the European best practice guidelines an autogenous arteriovenous fistula (AVF) is the access of first choice and was favored over arteriovenous grafts (AVG) whenever possible (3-5). In order of preference a radial-cephalic AVF (RC-AVF), brachial-cephalic AVF (BCAVF) or brachial-basilic AVF (BB-AVF) was created. A RC-AVF was created if the distal radial artery and cephalic vein had a diameter at DUS $>2 \mathrm{~mm}$. In case of smaller vessels or the presence of arterial or venous obstructions an alternative AVF was created instead of a RC-AVF. The use of tiny or diseased vessels, as determined by DUS, was avoided. An AVG was created if an AVF, in the opinion of the vascular surgeon, was likely to fail. The final decision as to the type of access was always made by the vascular surgeon who was involved in history taking and physical examination and who was aware of the DUS results. The surgeon was unaware of the CE-MRA results and the treatment was exclusively based on DUS derived parameters.

\section{Preoperative contrast-enhanced magnetic resonance angiography}

\section{MR imaging}

CE-MRA images were acquired without knowledge of DUS results. Patients were imaged in the supine position using multi-element phased array surface coils and a 1.5-Tesla MRI system (Philips Intera R9.1, Philips Medical Systems, Best, The Netherlands) (17). Venous access was established in a dorsal hand vein on both sides for contrast media injection. For each patient a maximum dose of $45 \mathrm{~mL}$ gadopentate dimeglumine (Gadolinium DTPA, Magnevist, Schering, Berlin, Germany) was used and special care was taken not to exceed $0.3 \mathrm{mmol} / \mathrm{kg}$. Acquired spatial resolution for thoracic and upper arm vessels was $1.00 \times 1.42 \times 2.50 \mathrm{~mm}^{3}$ and for forearm vessels $1.00 \times 1.43 \times 2.00 \mathrm{~mm}^{3}$. 


\section{Chapter 6}

\section{MR Image analysis}

CE-MRA images were read in random order by two observers with over 3 years experience in peripheral arterial CE-MRA. Review was done on the 3D CE-MRA scans, which exhibited the best selective arterial and venous vessel enhancement. Reviewers used maximum intensity projections (MIPs) and multiplanar reformations (MPRs) and were also required to review all source images.

Analysis of thoracic, upper arm and forearm vessels was done separately. Thoracic (station 1) vessel analysis included the aortic arch and the brachiocephalic, subclavian, and axillary arteries and the superior vena cava and the brachiocephalic, subclavian, and axillary veins. The upper arm (station 2) vessels included the brachial artery and the basilic and cephalic veins. Forearm (station 3) vessel analysis included the radial and ulnar arteries, and the basilic and cephalic veins. All vessels were assessed for the presence of stenoses and occlusions per station.

For determination of inter-observer agreement with regard to the degree of stenosis all arterial and venous segments as defined above were scored on a 4point scale (normal or minimal luminal irregularities, single stenosis with $>50 \%$ luminal diameter reduction, multiple stenoses with $>50 \%$ luminal diameter reduction or occlusion) based on the most severe lesion in each vessel segment. MR observers used multi-planar reformations and the full width half maximum algorithm for determination of the vessel diameters. The degree of luminal diameter reduction in a stenosis was determined by dividing the average diameter at the point of maximum stenosis by diameter of the closest adjacent normal vessel segment. A luminal diameter reduction of $>50 \%$ was considered clinically significant.

\section{Postoperative evaluation of hemodialysis vascular access function}

Patients were followed postoperatively until the vascular access was first used for hemodialysis and the functionality of the vascular access was recorded. A functional vascular access was defined as the ability to use the vascular access for two-needle hemodialysis, judged by a nephrologists, vascular surgeon and dialysis access nurse. Early failure was defined as vascular access thrombosis 
within 2 weeks after creation. Non-maturation was defined as a non-functional vascular access at 2 months after creation.

\section{Statistical analysis}

Inter-observer agreement for detection of stenoses and occlusions was calculated at patient level for each arterial and venous station separately. For analysis purposes, only the most severe stenosis in each vessel segment was taken into account. Inter-observer agreement for detection of stenosis and occlusions was determined by calculation of quadratic weighted kappa coefficients. The strength of agreement for the kappa coefficient was interpreted as suggested by Landis and Koch $(<0=$ poor, $0.01-0.20=$ slight, $0.21-0.40=$ fair, $0.41-0.60$ $=$ moderate, $0.61-0.80=$ substantial, and $0.81-1=$ almost perfect $)(20)$.

Cases of disagreement between both observers for detection of stenoses or occlusions were reviewed in a consensus reading.

The sensitivity, specificity, positive predictive value and negative predictive values of preoperative DUS and CE-MRA were calculated using clinical outcomes as standard of reference.

\section{Results}

\section{Study population}

From November 2003 until March 2006, a total of 114 patients were referred for hemodialysis vascular access creation and according to the intention to treat principle considered to be eligible for inclusion. A study flow diagram is shown in figure 1. A total of $17(15 \%)$ patients were excluded because of inability to undergo CE-MRA due to pacemaker $(n=9)$, obesity $(n=4)$, claustrophobia ( $=3)$ or spastic paralysis $(n=1)$. Another $15(13 \%)$ patients were also excluded because of refusal to participate $(n=4)$, renal function recovery $(n=3)$, conversion to peritoneal dialysis $(n=3)$, renal transplantation $n=1$, or death $(n=4)$. 


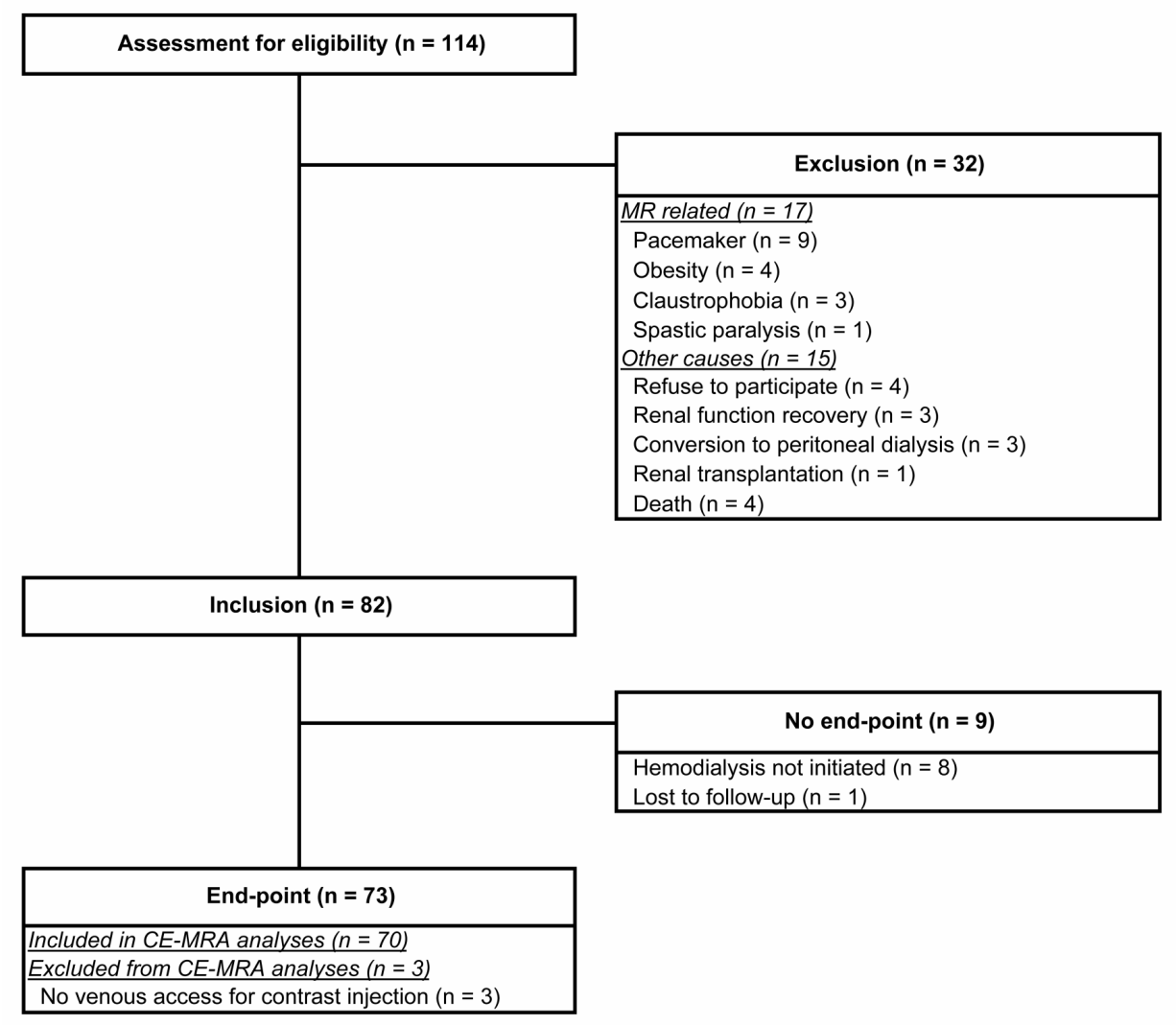

Figure 1: Study flow diagram showing the progress of patients and the profile of the study.

Eighty-two patients (male: $n=51$, female: $n=31$, mean age 68 years range 36 96 years) were included in the study. Forty-four (54\%) patients had no previous vascular access. Thirty-three patients had one or more previous central venous catheters (range 0-6) and 23 patients had undergone one or more previous vascular access procedures (range 0-6).

A vascular access was created in all 82 patients. Hemodialysis treatment was not initiated in 9 (11\%) patients by the end of the study because of stabilization of renal function ( $n=7$ ), renal function recovery $(n=1)$ and one patient was lost to follow-up. The functionality of the vascular accesses could therefore not be determined in these patients (RC-AVF: $n=4, B C-A V F: n=1, B B-A V F: n=1$. AVG: $\mathrm{n}=3$ ). 


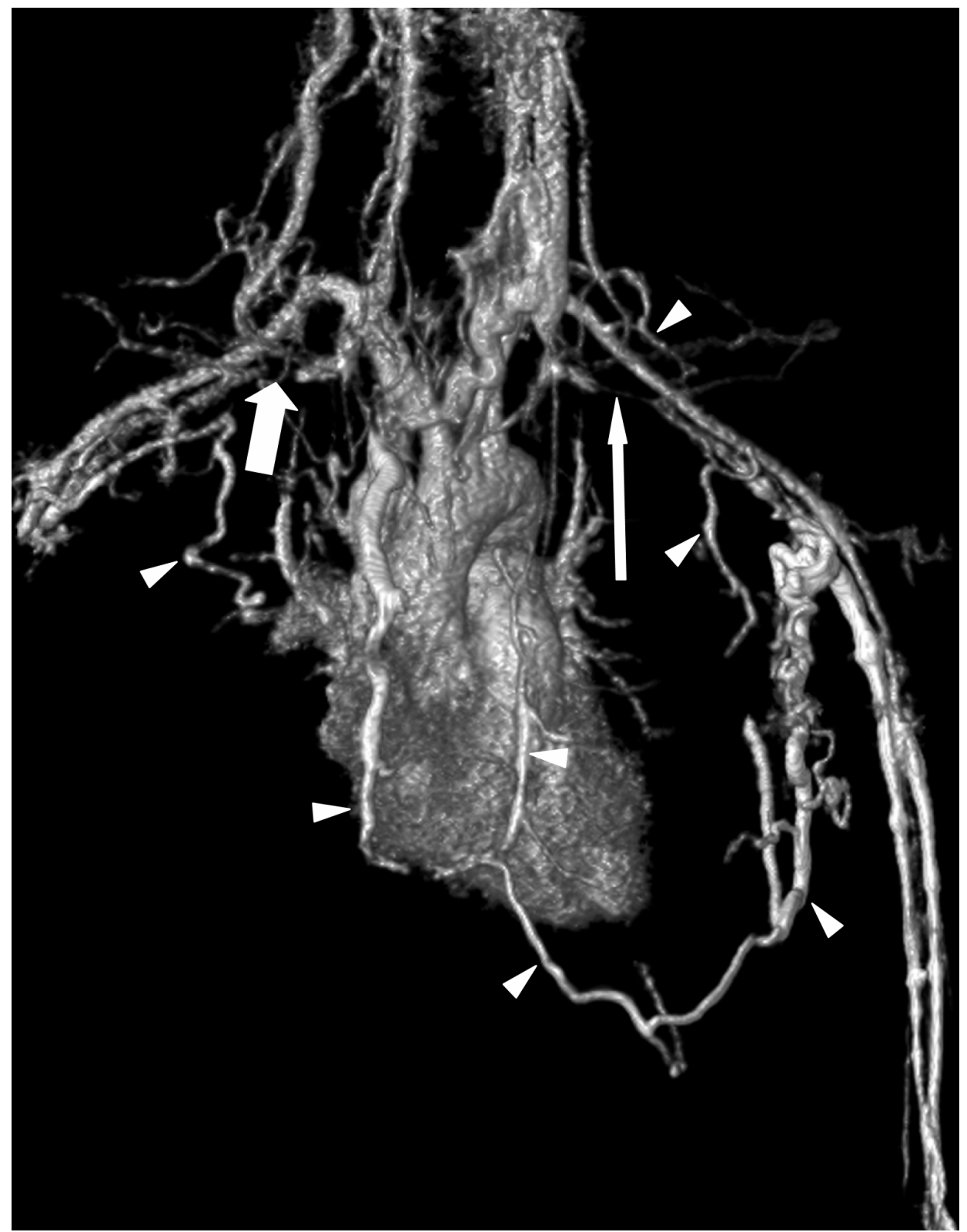

Figure 2: 3D CE-MRA volume rendered image of a patient with bilateral central venous occlusions (bold arrow and thin arrow). Note the many collateral veins present (arrowheads). 


\section{Chapter 6}

Subsequently, a total of 73 vascular accesses (AVF 67\%: 49/73, AVG 33\%: 24/73) were included for further analysis. Fifteen patients received a RC-AVF based on the preoperative DUS criteria. Other types of AVF were created in 34 patients (BC-AVF: 15, BB-AVF: 18, Thigh AVF: 1). In one patient, central venous occlusions and poor upper extremity veins were detected on DUS and CE-MRA (figure 2), and a thigh AVF instead of an upper extremity vascular access was created. AVG $(n=24)$ were created in the remaining group because of poor quality vessels, on DUS.

\section{Postoperative evaluation of hemodialysis vascular access function}

A functional vascular access was initially established in $70 \%(51 / 73)$ of all patients (RC-AVF 5/15 = 33\%, BC-AVF 11/15 = 73\%, BB-AVF 15/18 = 83\%, AVG $19 / 24=79 \%$, thigh AVF $1 / 1=100 \%$ ). Consequently, the positive predictive value of DUS for access function at 2 months was $70 \%$. Negative predictive value, sensitivity and specificity could not be calculated because surgery was DUS-guided. Access types created, their initial outcome and final outcomes $>2$ months postoperative, are listed in table 1 .

\section{Early failure and subsequent outcome}

Early failure occurred in $10 \%(7 / 73)$ of all cases. In five patients surgical thrombectomy combined with percutaneous transluminal angioplasty (PTA) or creation of a new arteriovenous anastomosis did not result in successful salvage and a new vascular access was created (RC-AVF: $n=1, B C-A V F: n=1$, BBAVF: $n=1$, AVG: $n=2$ ). Surgical thrombectomy combined with PTA resulted in successful salvage in remaining two patients (BC-AVF: $n=1$, AVG: $n=1$ ). Examples of preoperative CE-MRA findings associated with vascular access early failure are shown in figure 3.

\begin{tabular}{|c|c|c|c|c|c|c|c|}
\hline \multirow{3}{*}{$\begin{array}{l}\text { Maturation } \\
\text { Early failure }\end{array}$} & \multicolumn{2}{|c|}{$R C-A V F$} & \multirow{2}{*}{$\begin{array}{l}B C-A V F \\
73 \%(11 / 15)\end{array}$} & \multirow{2}{*}{$\begin{array}{l}B B-A V F \\
83 \%(15 / 18)\end{array}$} & \multirow{2}{*}{$\begin{array}{l}\text { AVG } \\
79 \%(19 / 24)\end{array}$} & \multicolumn{2}{|c|}{ Thigh-AVF } \\
\hline & $33 \%$ & $(5 / 15)$ & & & & $100 \%$ & $(1 / 1)$ \\
\hline & $7 \%$ & $(1 / 15)$ & $13 \% \quad(2 / 15)$ & $6 \% \quad(1 / 18)$ & $13 \% \quad(3 / 24)$ & $0 \%$ & $(0 / 1)$ \\
\hline on & $60 \%$ & $(9 / 15)$ & $(2 / 15)$ & $(2 / 18)$ & $(2 / 24)$ & $0 \%$ & $(0 / 1)$ \\
\hline & $89 \%$ & $(8 / 9)$ & $(3 / 4)$ & $(2 / 3)$ & $100 \% \quad(3 / 3)$ & & $(0 / 0)$ \\
\hline
\end{tabular}

Table 1: Accesses created, salvage procedures and clinical outcome. 

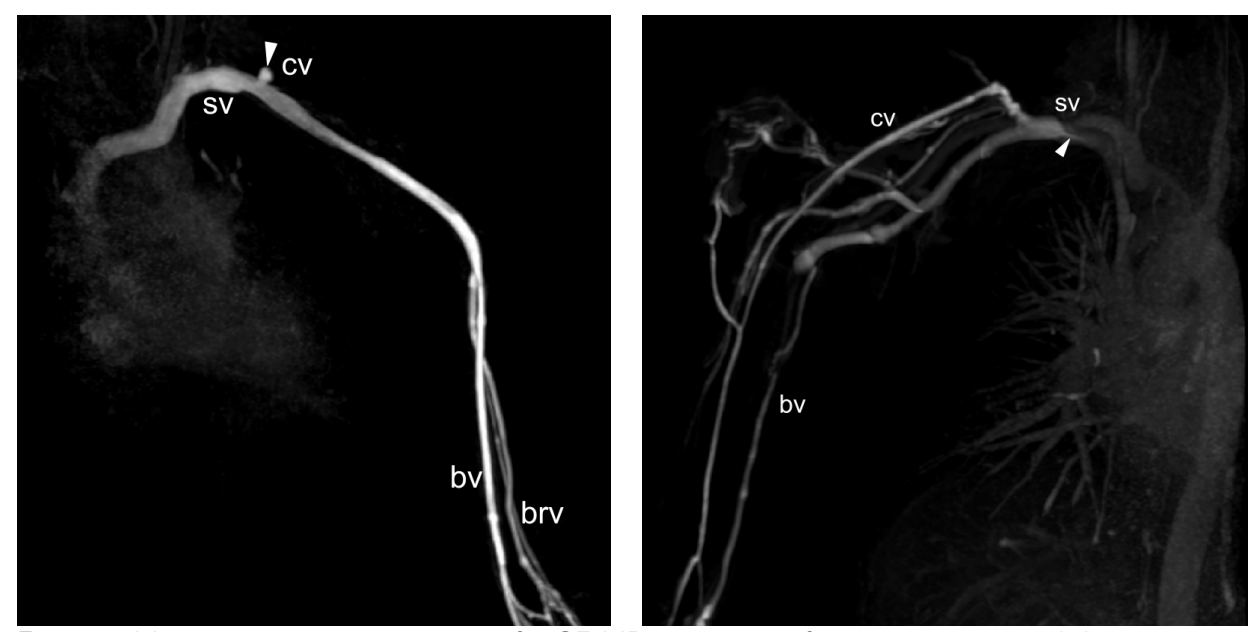

Figure 3: Maximum intensity projection of a CE-MR angiogram of upper extremity and thoracic veins, (A) of a patient who received a BC-AVF that failed within the first days after creation. The arrowheads point at the occluded cephalic vein. and (B) of a patient who received a BB-AVF that failed within the first days after creation. A small caliber basilic vein and subclavian vein stenosis were detected by CE-MRA. (bv = basilic vein, brv = brachial vein, $\mathrm{cv}=$ cephalic vein, $\mathrm{sv}=$ subclavian vein).

\section{Non-maturation and subsequent outcome}

Non-maturation occurred in $21 \%(15 / 73)$ of all cases. One patient received a renal transplant and no salvage procedure was therefore performed. Salvage procedures for vascular accesses that failed to mature were successful in all cases. Salvage procedures for RC-AVF non-maturation were accessory vein ligation $(n=4)$, PTA of venous stenoses $(n=4)$ or a combination of both $(n=$ 2). Two non-matured BC-AVF were salved successfully by PTA of a venous stenosis $(n=1)$ or ligation of an accessory vein in addition to creation of a venous patch $(n=1)$. Non-maturation of BB-AVFs was successfully treated by venous PTA $(n=1)$ or arterial PTA $(n=1)$. Two patients in whom an AVG failed to mature became functional after PTA of venous outflow stenoses. In figures 4 and 5 examples are shown of preoperative CE-MRA findings associated with vascular access non-maturation.

After salvage procedures, $92 \%$ (67/73) of all accesses were functional by the end of this study (RC-AVF: $13 / 15=87 \%$, BC-AVF: $14 / 15=93 \%$, BB-AVF: $17 / 18=$ $94 \%$ and AVG: $22 / 24=92 \%$, Thigh AVF: $1 / 1=100 \%$ ). 


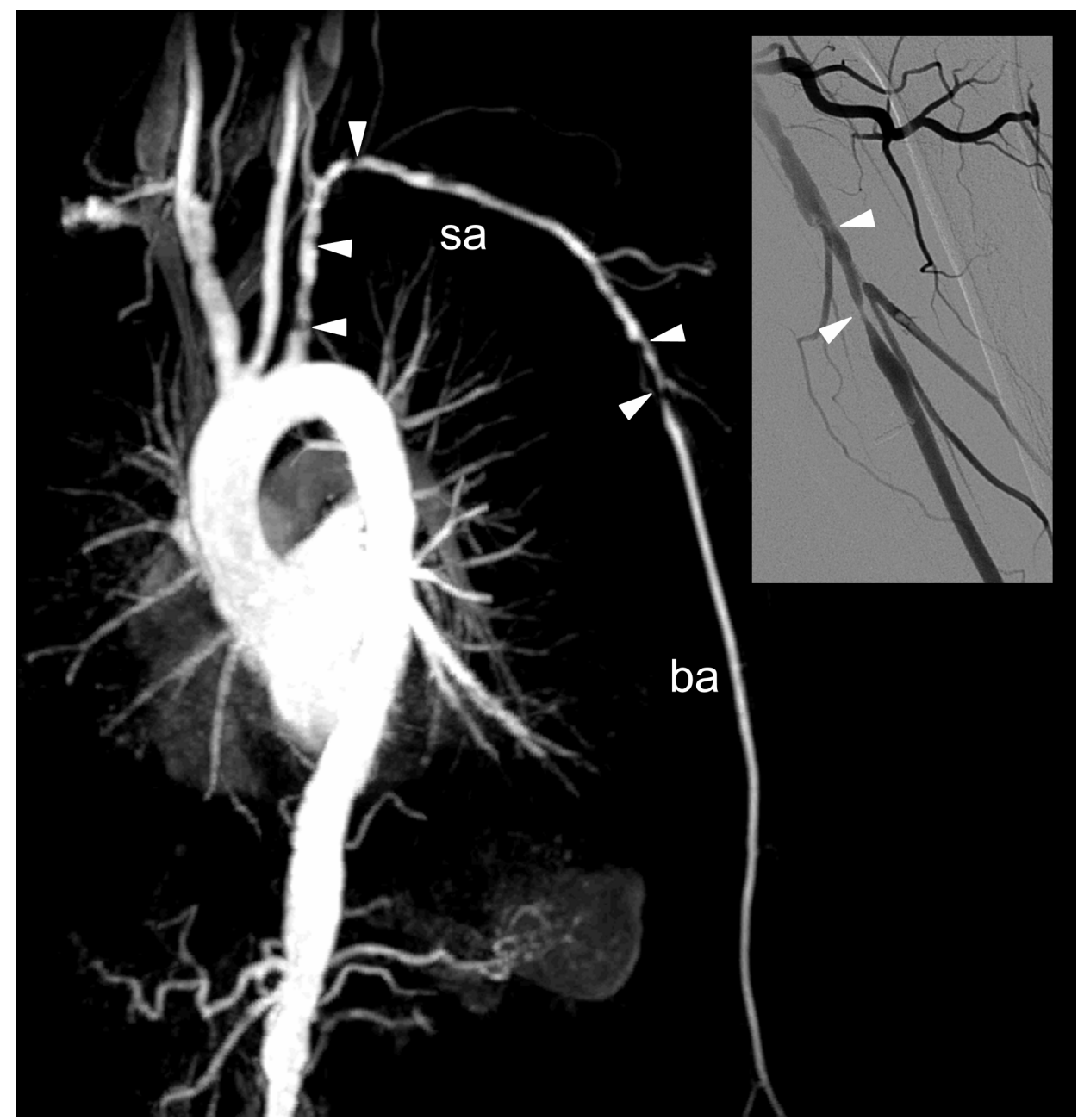

Figure 4: Maximum intensity projection of a CE-MR angiogram of upper extremity and thoracic arteries of a patient who received a BB-AVF that failed to mature. Arterial stenosis (arrowheads) was detected by CE-MRA and confirmed by DSA (inlay). (ba = brachial artery, sa = subclavian artery) 

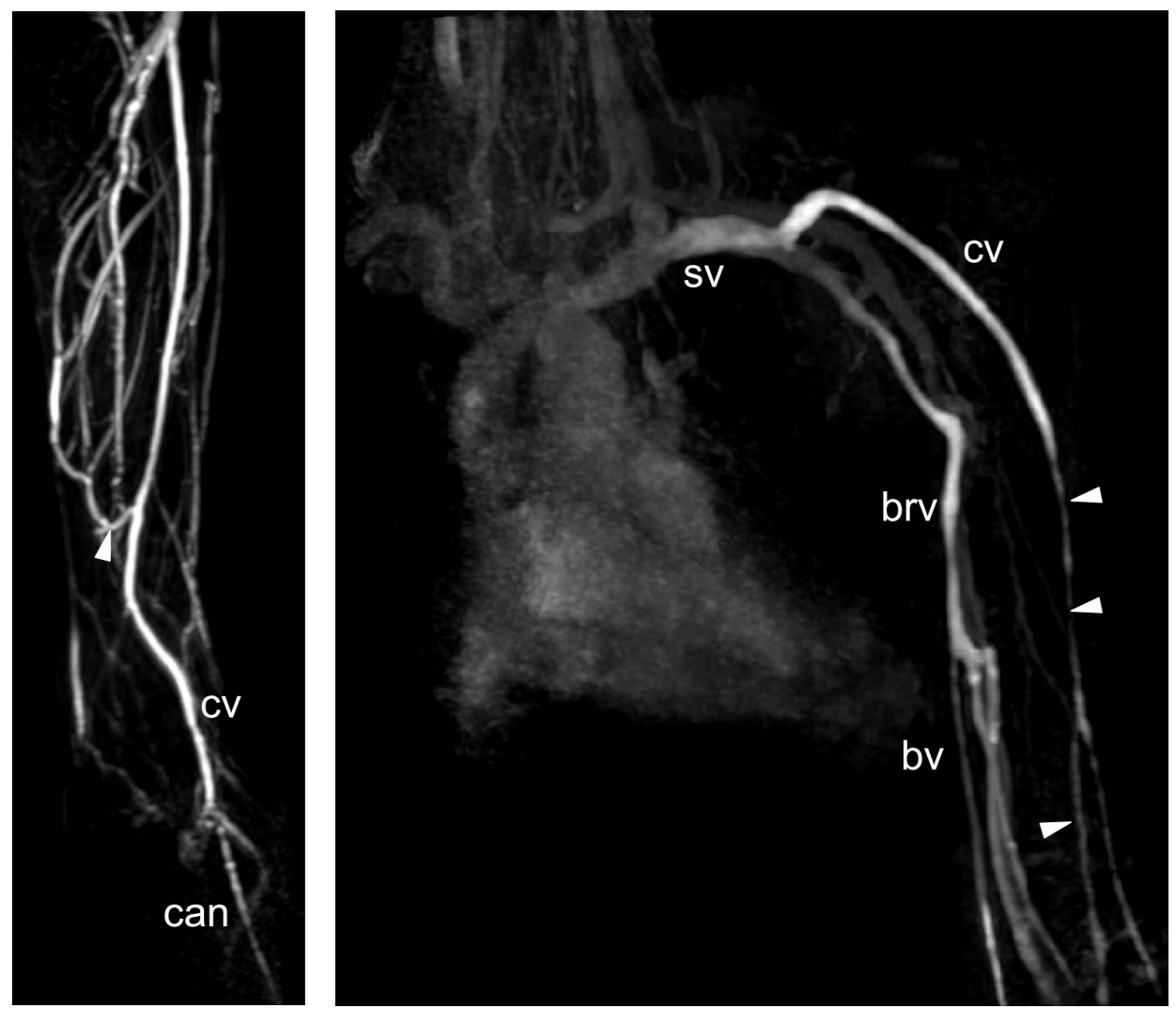

Figure 6: (left) Maximum intensity projection of a CE-MR angiogram of forearm veins of a patient who received a RC-AVF that failed to mature due to a large caliber accessory vein (arrowhead). The RCAVF matured successfully after surgical ligation of this accessory vein. (can = intravenous canula, $\mathrm{cr}$ = cephalic vein), (right) maximum intensity projection of a CE-MR angiogram of upper extremity and thoracic veins of a patient who received a BC-AVF that failed to mature. CE-MRA showed a cephalic vein (arrowheads) that was tiny when compared with the basilic vein. On DUS the cephalic vein was considered of good caliber and quality whereas the basilic vein could not be depicted by DUS. The BC-AVF became functional after multiple dilatations of the cephalic vein. (bv = basilic vein, $\mathrm{cv}=$ cephalic vein, sv = subclavian vein). 


\section{Preoperative contrast-enhanced magnetic resonance angiography}

\section{MR imaging}

A total of 70 MR-arteriograms of the proximal and distal arteries including the palmar arch were available for 70 out of 73 patients. In 3 patients, ipsi-lateral and contra-lateral intravenous access for contrast media injection could not be established. All three patients received an AVG because of poor venous quality. One of these three AVG failed to mature due to a venous outflow stenosis that was successfully treated by PTA. Because these patients were not successfully assessed by CE-MRA, they were excluded from further analysis.

Arterial and venous stenoses and occlusions as detected by CE-MRA are listed in table 2.

\section{MR-image analysis}

Inter-observer agreement was almost perfect for detection of stenosis and obstructions for thoracic, upper arm and forearm arteries and upper arm veins and moderate for thoracic veins and forearm veins (table 3 ).

$\begin{array}{lccc} & \text { Stenosis (n) } & \text { Multiple-stenosis ( } n \text { ) } & \text { Occlusion }(n) \\ \text { Arterial } & 0 & 0 & 0 \\ \text { Aortic arch } & 1 & 0 & 0 \\ \text { Subclavian artery } & 0 & 1 & 0 \\ \text { Axillary artery } & 1 & 1 & 0 \\ \text { Brachial artery } & 1 & 1 & 3 \\ \text { Radial artery } & 0 & 0 & 5 \\ \text { Ulnar artery } & & & \\ \text { Venous } & 0 & 0 & 1 \\ \text { Superior vena cava } & 1 & 0 & 1 \\ \text { Subclavian vein } & 2 & 1 & 0 \\ \text { Axillary vein } & 1 & 0 & 24 \\ \text { Cephalic vein (proximal) } & 0 & 1 & 13 \\ \text { Basilica vein (proximal) } & 2 & 7 & 1 \\ \text { Cephalic vein (distal) } & 0 & 0 & \\ \text { Median cubital vein } & & & \end{array}$

Table 2: CE-MRA findings $(n=70), n$ : number of patients with a stenois or multiple stenoses or occlusion per vessel. 
Kappa coefficient $( \pm 95 \% \mathrm{Cl})$

Arterial

Thorax

Upper arm

Forearm

Venous

Thorax

Upper arm

Forearm

$$
\begin{aligned}
& 0.91(0.69-1.00) \\
& 0.89(0.61-1.00) \\
& 0.90(0.77-1.00)
\end{aligned}
$$

$$
\begin{aligned}
& 0.80(0.52-1.00) \\
& 0.96(0.90-1.00) \\
& 0.76(0.59-1.00)
\end{aligned}
$$

\section{Agreement}

Almost perfect $(0.81-1.00)$

Almost perfect $(0.81-1.00)$

Almost perfect $(0.81-1.00)$

Substantial (0.61-0.80)

Almost perfect $(0.81-1.00)$

Substantial (0.61-0.80)

Table 3: The inter-observer agreement of upper extremity CE-MRA for detection of stenosis and occlusions and for assessment of the arterial palmar arch patency $(n=70)$.

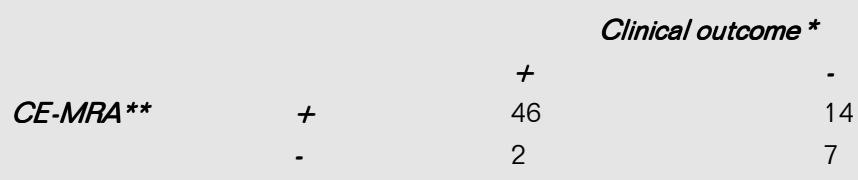

Table 4: CE-MRA predictions and clinical outcome, ${ }^{*}=$ clinical outcome based on DUS guided surgical treatment plan, ${ }^{* *}=$ clinical outcome as predicted by CE-MRA, $+=$ maturation, $-=$ early access failure or non-maturation.

\section{Value of CE-MRA for prediction of early failure and non-maturation}

Preoperative CE-MRA predictions and subsequent clinical outcome of the 69 patients who received an upper extremity hemodialysis access are shown in table 4. CE-MRA had a sensitivity, specificity, positive predictive value and negative predictive value of $96 \%, 33 \%, 77 \%$ and $88 \%$ respectively. CE-MRA successfully predicted early failure or non-maturation in $33 \%(7 / 21)$ of the cases.

Both DUS and CE-MRA wrongly predicted successful access creation in a total of 14 patients with access early failure or non-maturation. These cases were therefore considered false positive results that caused a low CE-MRA specificity of $33 \%$ for prediction of vascular access outcome. Causes of early failure and non-maturation were venous stenosis $(n=9)$, accessory veins $(n=4)$ or a combination of both $(n=1)$ in these patients. 
DUS correctly predicted successful access creation in two patients in whom CEMRA predicted access early failure or non-maturation. An arterial stenosis was detected by CE-MRA and not by DUS in one of these two patients who received a functioning AVG. This preoperative CE-MRA finding was therefore considered false negative result, in the light of access early failure and non-maturation. However, this patient developed steal symptoms a few months after AVG creation and the arterial stenosis was confirmed by DSA. In retrospect, this preoperative CE-MRA finding was clinically relevant although not for prediction of initial access function. In another patient with a previous ipsilateral RC-AVF, a brachio-cephalic vein occlusion was detected by CE-MRA and not by DUS (figure 7). This patient received a BC-AVF that matured successfully despite the brachio-cephalic vein occlusion. The brachio-cephalic vein occlusion was therefore also considered a false negative result.

\section{Discussion}

With this study we have shown that CE-MRA is a reliable tool for detection of stenoses and occlusion in the upper extremity arteries and veins. CE-MRA proved to be a potentially valuable tool in the workup prior to hemodialysis access creation to improve vascular access outcome.

Hemodialysis access early failure and non-maturation are frequently encountered serious complications in patients requiring hemodialysis treatment because they necessitate additional interventions and the use of central venous catheters to gain vascular access for hemodialysis. Central venous catheters frequently lead to life threatening infections and deterioration of central veins. The use of central venous catheters is therefore strongly discouraged by the NKF K-DOQI and European guidelines $(3,4)$. The current study was motivated by the fact that reported early failure and non-maturation rates of newly created hemodialysis vascular access remain high despite the clinical application of preoperative DUS $(6,13,15,16)$. Because CE-MRA is highly accurate for assessment of the upper extremity vasculature and in particular central veins, we hypothesized that CEMRA might be a valuable technique in the workup prior to hemodialysis vascular access creation to better predict initial access outcome (21-26). CE-MRA showed substantial to almost perfect inter-observer agreement for detection of upper extremity arterial and venous stenoses in this study. 


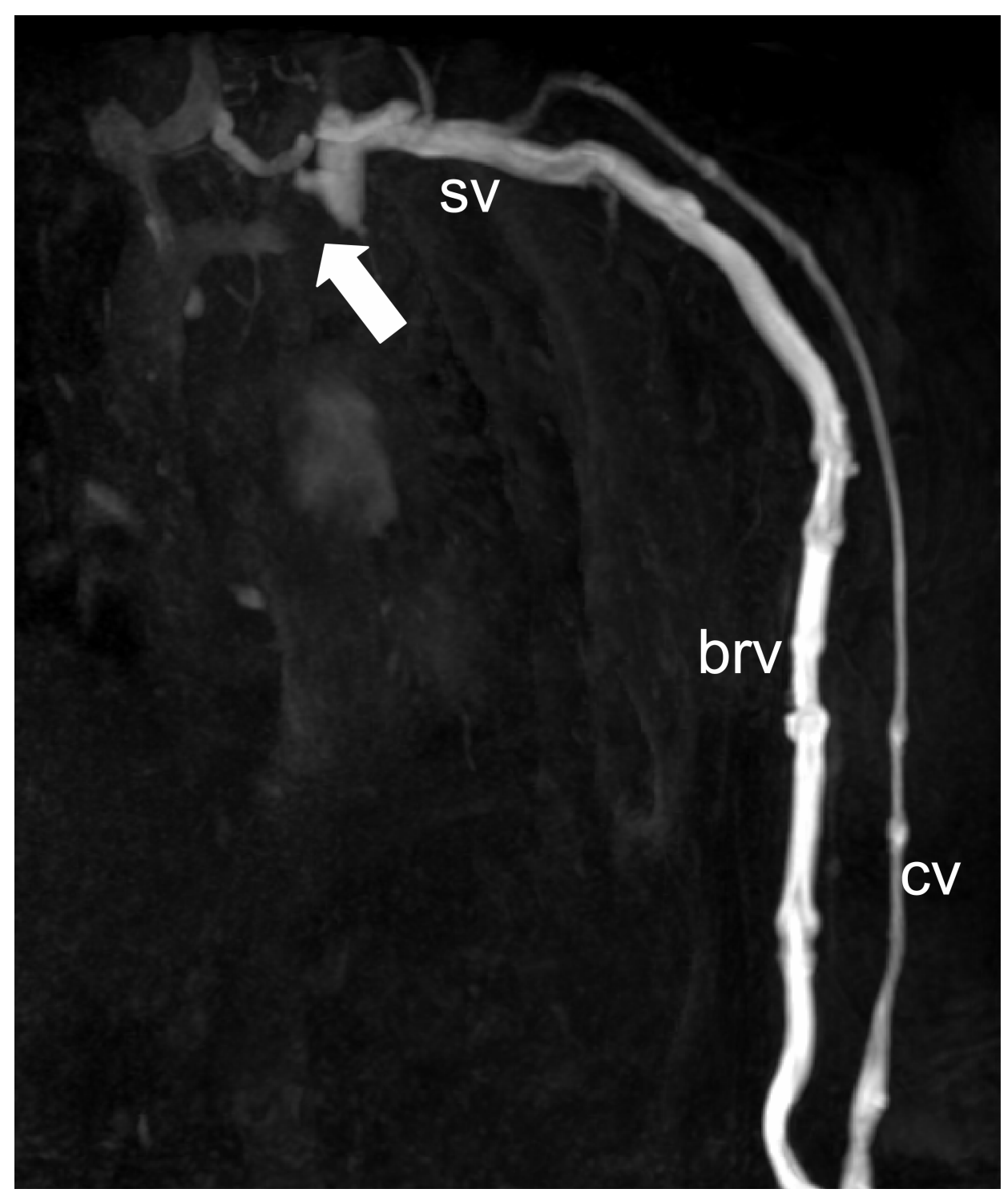

Figure 8: Anteroposterior maximum intensity projection of a CE-MR angiogram of upper extremity and thoracic veins of a patient with an occluded brachio-cephalic vein (arrow). (brv = brachial vein, $\mathrm{cv}=$ cephalic vein and $\mathrm{sv}=$ subclavian vein) 
The proportion of RC-AVF that failed to mature was strikingly high in this study. Early failure and non-maturation rates in this study are in agreement with the literature $(6,13,15,16)$. The current study again underscores that the current standard of care, which relies on diameter measurements and detection of arterial and venous obstructions by DUS alone, seems incapable of further reducing early failure and non-maturation.

CE-MRA turned out to be of additional value and successfully predicted early failure and non-maturation in 33\% of the cases. If CE-MRA had been the primary treatment-guiding modality, this would have potentially resulted in lower early failure and non-maturation rates compared to DUS. Examples of the added value of CE-MRA are a case of subclavian artery stenosis and a case of central venous occlusion which were detected by CE-MRA but were not seen at DUS. These abnormalities did affect long-term access function. However, short-term access function was the primary interest of the current study.

Central venous obstructions can lead to low access flow and venous hypertension and are therefore believed to be associated with poor vascular access outcome. However, this does not hold for all patients as evidenced by the patient with the high flow access despite a central venous occlusion (figure 8). This patient had a previous RC-AVF in the same arm and it is likely that collateral draining veins, as seen on the preoperative CE-MRA had already developed before BC-AVF creation and that the central venous obstruction therefore did not effect BC-AVF maturation.

Early failure and non-maturation were due to venous outflow stenosis or occlusions in all except one case in whom an arterial stenosis was the cause of non-maturation. Access early failure and non-maturation could potentially have been anticipated based on relevant CE-MRA findings in 33\% (7/21) and not in the remaining $67 \%(15 / 21)$ cases. DUS and CE-MRA, however, did show adequate arterial and venous diameters and no arterial or venous stenosis or obstruction in this $67 \%$ of patients. In this group of patients venous stenoses and large caliber accessory veins turned out to be the cause of early failure and nonmaturation. We suggest that these venous stenoses developed after vascular access creation because they were not detected by DUS or CE-MRA preoperatively. The most likely reason for this is the almost instantaneous switch 
from a low-flow to a high-flow environment after creation of the arteriovenous anastomosis. Based on the mainly morphological parameters we assessed, early failure and non-maturation due to venous stenoses could therefore not have been anticipated studying these patients.

Accessory veins, on the other hand, were the cause of non-maturation in a substantial group of patients receiving a RC-AVF, the type of access with the highest non-maturation rate in this study and in literature. However, accessory veins have to date not been reported as a preoperative indicator of postoperative poor access outcome. In the current study we found that ligation of large caliber accessory veins resulted in successful maturation in $40 \%(4 / 10)$ of all nonmatured RC-AVF. One of the most important insights is therefore that preoperative detection of large caliber accessory veins and subsequent selective ligation during initial RC-AVF creation has the potential to result in a reduction of RC-AVF non-maturation rates. Further prospective studies will have to be conducted to test this hypothesis.

Early referral, as recommended by the NKF K-DOQI and European guidelines, resulted in creation of a vascular access that was never used in $10 \%(8 / 82)$ of patients because hemodialysis treatment was not initiated due to renal function recovery or stabilization. One might question if this is an acceptable consequence of early referral. The current study has limitations. An important caveat is that CE-MRA is not a suitable test for all patients with ESRD. Of the potentially eligble 114 patients, 17 (15\%) were excluded from participation in the current study because of MR-safety precautions or other MR-related causes. This is an important limitation because patients with pacemakers are more likely to have central venous abnormalities. However, recent reports suggest that MR imaging is a safe procedure in patients with pacemakers or implantable cardioverterdefibrilattors under controlled conditions by taking both MR- and device precautions (27-29).

Achieving venous access for contrast media injection was cumbersome in a few patients and is therefore considered to be a limitation as well. A second important limitation relates to the study design. Although the current study prospectively compared DUS and CE-MRA, treatment was exclusively based on DUS. This design was chosen because at the onset of the study it was unknown whether 


\section{Chapter 6}

CE-MRA would be able to detect relevant arterial stenoses and occlusions that DUS could not. Although the relevance of the lesions visualized exclusively at CE-MRA was confirmed over the course of the study, the ability of CE-MRA to reduce non-maturation and early failure remains to be confirmed in a randomized clinical trial.

When used intravenously, no detectable nephrotoxicity has been reported and the rates of adverse events are extremely low (30-32). However, recently concerns have arisen regarding the accumulation of free gadolinium in patients with renal failure (33). During the last decade approximately 200 cases of nephrogenic systemic fibrosis (NSF), previously known as nephrogenic fibrosing dermopathy, have been reported worldwide (34). The reported clinical sings and symptoms of NSF are sub acute progressive swelling of extremities followed by more proximal involvement and sever skin induration, pain, muscle restlessness and loss of skin flexibility. NSF can lead to serious physical disability and wheelchair requirement (34). The incidence of NSF is low and the pathophysiology is unknown (34). To date, only gadolinium in the form of gadodiamide (Omniscan) has been reported to be associated with NSF (34). Gadopentate dimeglumine (Magnevist, Schering, Berlin, Germany), the contrast medium used in this study has not been reported to be associated with nephrogenic systemic fibrosis and none of the included patients developed NSF symptoms.

In conclusion, early failure and non-maturation of newly created vascular accesses is a serious and frequently encountered complication. CE-MRA enables detection of upper extremity arterial and venous stenosis and occlusions, related with vascular access early failure and non-maturation, with substantial to almost perfect inter-observer agreement. CE-MRA is a valuable tool for prediction of vascular access early failure and non-maturation with a better predictive value for prediction of vascular access outcome than DUS. 


\section{References}

1. Grassmann A, Gioberge S, Moeller S, Brown G. ESRD patients in 2004: global overview of patient numbers, treatment modalities and associated trends. Nephrol Dial Transplant 2005;20(12):2587-93.

2. Moeller S, Gioberge S, Brown G. ESRD patients in 2001: global overview of patients, treatment modalities and development trends. Nephrol Dial Transplant 2002;17(12):2071-6.

3. Tordoir JH, Mickley V. European guidelines for vascular access: clinical algorithms on vascular access for haemodialysis. Edtna Erca J 2003;29(3):131-6.

4. III. NKF-K/DOQI Clinical Practice Guidelines for Vascular Access: update 2000. Am J Kidney Dis $2001 ; 37$ (1 Suppl 1):S137-81.

5. Clinical practice guidelines for vascular access. Am J Kidney Dis 2006;48 Suppl 1:S176-247.

6. Allon M, Lockhart ME, Lilly RZ, Gallichio MH, Young CJ, Barker J, et al. Effect of preoperative sonographic mapping on vascular access outcomes in hemodialysis patients. Kidney Int $2001 ; 60(5): 2013-20$.

7. Ascher E, Gade P, Hingorani A, Mazzariol F, Gunduz Y, Fodera M, et al. Changes in the practice of angioaccess surgery: impact of dialysis outcome and quality initiative recommendations. J Vasc Surg 2000;31(1 Pt 1):84-92.

8. Silva MB, Jr., Hobson RW, 2nd, Pappas PJ, Jamil Z, Araki CT, Goldberg MC, et al. A strategy for increasing use of autogenous hemodialysis access procedures: impact of preoperative noninvasive evaluation. J Vasc Surg 1998;27(2):302-7; discussion 307-8.

9. Wong V, Ward R, Taylor J, Selvakumar S, How TV, Bakran A. Factors associated with early failure of arteriovenous fistulae for haemodialysis access. Eur J Vasc Endovasc Surg 1996;12(2):207-13.

10. Malovrh M. Non-invasive evaluation of vessels by duplex sonography prior to construction of arteriovenous fistulas for haemodialysis. Nephrol Dial Transplant 1998;13(1):125-9.

11. Malovrh M. Native arteriovenous fistula: preoperative evaluation. Am J Kidney Dis 2002;39(6):1218-25.

12. Robbin ML, Gallichio MH, Deierhoi MH, Young CJ, Weber TM, Allon M. US vascular mapping before hemodialysis access placement. Radiology 2000;217(1):83-8.

13. Miller PE, Tolwani A, Luscy CP, Deierhoi MH, Bailey R, Redden DT, et al. Predictors of adequacy of arteriovenous fistulas in hemodialysis patients. Kidney Int 1999;56(1):275-80.

14. Palder SB, Kirkman RL, Whittemore AD, Hakim RM, Lazarus JM, Tilney NL. Vascular access for hemodialysis. Patency rates and results of revision. Ann Surg 1985;202(2):235-9.

15. Rooijens PP, Tordoir JH, Stijnen T, Burgmans JP, Smet de AA, Yo TI. Radiocephalic wrist arteriovenous fistula for hemodialysis: meta-analysis indicates a high primary failure rate. Eur $\mathrm{J}$ Vasc Endovasc Surg 2004;28(6):583-9. 


\section{Chapter 6}

16. Tordoir JH, Rooyens P, Dammers R, van der Sande FM, de Haan M, Yo TI. Prospective evaluation of failure modes in autogenous radiocephalic wrist access for haemodialysis. Nephrol Dial Transplant 2003;18(2):378-83.

17. Planken RN, Tordoir JHM, de Haan MW, Backes WH, van Engelshoven JMA, Leiner T. Selective MR-Arteriography and Venography Compared to Ultrasonography of the Entire Upper Extremity prior to Arteriovenous Fistula Placement for Hemodialysis. In: Radiological Society of Northern America scientific assembly and annual meeting program; 2005; Oak Brook, IL; 2005. p. 407.

18. Planken RN, Keuter XH, Kessels AG, Hoeks AP, Leiner T, Tordoir JH. Forearm cephalic vein cross sectional area changes associated with incremental venous congestion pressure analysis in young healthy male volunteers: towards a reproducible and standardized vein mapping protocol. J Vasc Surg 2006; 44(2): 353-8.

19. Planken RN, Keuter XH, Hoeks AP, Kooman JP, van der Sande FM, Kessels AG, et al. Diameter measurements of the forearm cephalic vein prior to vascular access creation in end-stage renal disease patients: graduated pressure cuff versus tourniquet vessel dilatation. Nephrol Dial Transplant 2006;21(3):802-6.

20. Landis JR, Koch GG. The measurement of observer agreement for categorical data. Biometrics 1977;33(1):159-74.

21. Ruehm SG, Zimny K, Debatin JF. Direct contrast-enhanced 3D MR venography. Eur Radiol 2001;11(1):102-12.

22. Ruehm SG. MR venography. Eur Radiol 2003;13(2):229-30.

23. Shinde TS, Lee VS, Rofsky NM, Krinsky GA, Weinreb JC. Three-dimensional gadoliniumenhanced MR venographic evaluation of patency of central veins in the thorax: initial experience. Radiology 1999;213(2):555-60.

24. Li W, David V, Kaplan R, Edelman RR. Three-dimensional low dose gadolinium-enhanced peripheral MR venography. J Magn Reson Imaging 1998;8(3):630-3.

25. Goyen M, Barkhausen J, Kuehl H, Goehde SC, Kroger K, Bosk S, et al. [Contrast-enhanced 3D MR venography of central thoracic veins: preliminary experience]. Rofo Fortschr Geb Rontgenstr Neuen Bildgeb Verfahr 2001;173(4):356-61.

26. Thornton MJ, Ryan R, Varghese JC, Farrell MA, Lucey B, Lee MJ. A three-dimensional gadolinium-enhanced MR venography technique for imaging central veins. AJR Am J Roentgenol 1999;173(4):999-1003.

27. Faris OP, Shein M. Food and Drug Administration perspective: Magnetic resonance imaging of pacemaker and implantable cardioverter-defibrillator patients. Circulation 2006;114(12):1232-3.

28. Nazarian S, Roguin A, Zviman MM, Lardo AC, Dickfeld TL, Calkins H, et al. Clinical utility and safety of a protocol for noncardiac and cardiac magnetic resonance imaging of patients with permanent pacemakers and implantable-cardioverter defibrillators at 1.5 tesla. Circulation 2006;114(12):1277-84.

29. Sommer T, Naehle CP, Yang A, Zeijlemaker V, Hackenbroch M, Schmiedel A, et al. Strategy for safe performance of extrathoracic magnetic resonance imaging at 1.5 tesla in the presence of 
cardiac pacemakers in non-pacemaker-dependent patients: a prospective study with 115 examinations. Circulation 2006;114(12):1285-92.

30. Cochran ST, Bomyea K, Sayre JW. Trends in adverse events after IV administration of contrast media. AJR Am J Roentgenol 2001;176(6):1385-8.

31. Prince MR, Arnoldus C, Frisoli JK. Nephrotoxicity of high-dose gadolinium compared with iodinated contrast. J Magn Reson Imaging 1996;6(1):162-6.

32. Tombach B, Bremer C, Reimer P, Kisters K, Schaefer RM, Geens V, et al. Renal tolerance of a neutral gadolinium chelate (gadobutrol) in patients with chronic renal failure: results of a randomized study. Radiology $2001 ; 218(3): 651-7$.

33. Shellock FG, Kanal E. Safety of magnetic resonance imaging contrast agents. J Magn Reson Imaging 1999;10(3):477-84.

34. Marckmann P, Skov L, Rossen K, Dupont A, Damholt MB, Heaf JG, et al. Nephrogenic systemic fibrosis: suspected causative role of gadodiamide used for contrast-enhanced magnetic resonance imaging. J Am Soc Nephrol 2006;17(9):2359-62. 



\section{Chapter 7}

Accessory veins and radial-cephalic arteriovenous fistula non-maturation: a prospective analysis using contrastenhanced magnetic resonance angiography

R.N. Planken, A.G. Kessels, T. Leiner, J.P. Kooman, F.M. van der Sande, J.H.M. Tordoir

Submitted 


\section{Abstract}

Purpose: To prospectively determine the value of accessory vein caliber for prediction of radial-cephalic arteriovenous fistula (RC-AVF) non-maturation.

Methods: RC-AVFs were created in 15 consecutive patients. Preoperative duplex ultrasound criteria for RC-AVF creation were a radial artery and cephalic vein diameter $>2 \mathrm{~mm}$ in the absence of arterial inflow or venous outflow stenoses or occlusions. Preoperative contrast-enhanced magnetic resonance angiography, in addition to the duplex ultrasound assessment was performed for detection of arterial and venous stenoses, occlusions and accessory veins. The location and caliber of accessory veins was determined. Access function post operative was monitored and all interventions for RC-AVF non-maturation were recorded. The value of accessory vein caliber for prediction of radial-cephalic arteriovenous fistula (RC-AVF) non-maturation was evaluated.

Results: Non-maturation occurred in 10 (67\%) out of 15 RC-AVFs. Large caliber accessory veins $(n=4)$, venous stenosis $(n=3)$, both $(n=2)$ were associated with RC-AVF non-maturation. Large caliber accessory veins were significant predictors for $\mathrm{RC}-\mathrm{AVF}$ non-maturation $(P=0.01)$. Preoperatively detected accessory veins with a diameter $>70 \%$ of the cephalic vein diameter, had a sensitivity, specificity, PPV and NPV of $80 \%, 100 \%, 100 \%$ and $91 \%$, respectively. For a cut-off value of $75 \%$ the sensitivity, specificity, PPV and NPV of $100 \%$, $90 \%, 83 \%$ and $100 \%$, respectively. Accessory vein ligation and dilatation of venous stenosis resulted in an overall salvage success-rate of $89 \%(8 / 9)$.

Conclusion: Large caliber accessory veins are associated with RC-AVF nonmaturation. Ligation of large caliber accessory veins is a successful salvage procedure in a substantial group of patients. Future studies need to determine if ligation of large caliber accessory veins during initial RC-AVF creation will reduce non-maturation rates. 


\section{Introduction}

Autogenous arteriovenous fistulas and the radial-cephalic arteriovenous fistula (RC-AVF) in particular, are considered the first choice vascular access for longterm hemodialysis treatment because of fewer complications when compared to arteriovenous grafts or central venous catheters (1). A major drawback of AVF is the incidence of early failure or non-maturation. Early failure can be defined as access thrombosis within two weeks after creation whereas non-maturation can be described as insufficient access flow and caliber increase which makes the vascular access unsuitable for hemodialysis access use. Preoperative assessment has been reported to enable prediction and thereby prevention of access early failure and non-maturation. However, despite preoperative upper extremity vessel assessment by duplex ultrasound (DUS), up to 50\% of all newly created RC-AVFs fail to mature $(2,3)$. Access early failure and non-maturation are serious complications because they necessitate additional interventions and the use of central venous catheters which increases the risk of future vascular access failure (4). In the occurrence of non-maturation, successful salvage can be achieved in up to $92 \%$ of the cases by either dilatation of stenosis or ligation of accessory veins or both(5). The significance of accessory veins in the light of RC-AVF nonmaturation, however, remains matter of discussion (5-9). It has been reported that a postoperative venous flow $<500 \mathrm{~mL} / \mathrm{min}$ and diameter $<4 \mathrm{~mm}$ are associated with non-maturation of AVFs (9). Accessory veins with a diameter $>70 \%$ of the cephalic vein diameter result in a substantial alteration in AVF flow distribution and decrease in cephalic vein flow (10). We hypothesize that accessory veins may lead to RC-AVF non-maturation because of a decrease in cephalic vein flow. Furthermore, preoperative assessment of large caliber accessory veins might therefore enable prediction of RC-AVF non-maturation. The purpose of this study was to prospectively determine the value of accessory vein caliber for prediction of RC-AVF non-maturation.

\section{Materials and methods}

According to the NKF K-DOQI vascular access guidelines and the European best practice guidelines all patients requiring a new hemodialysis vascular access were preoperatively assessed by DUS at our institution(1, 11). Our institutional policy 
is to create RC-AVFs in patients with a radial artery and cephalic vein diameter $>2 \mathrm{~mm}$ in the absence of arterial inflow or venous outflow stenoses or occlusions.

All patients underwent contrast-enhanced magnetic resonance angiography (CEMRA) prior to RC-AVF creation, in addition to the standard DUS. The medical ethics committee of the institution approved the study and all patients signed informed consent prior to inclusion.

\section{Contrast-enhanced magnetic resonance angiography}

Contrast-enhanced MR-images were acquired using a commercially available 1.5T MR scanner (Intera R9.1, Philips Medical Systems, Best, The Netherlands). Acquired spatial resolutions for thoracic and upper arm vessels were $1.00 \times 1.43 \times 2.00 \mathrm{~mm}^{3}$ and for forearm vessels $1.00 \times 1.42 \times 2.50 \mathrm{~mm}^{3}(12)$.

One observer analyzed the CE-MRA images using the dynamic 3D CE-MRA scan which exhibited the best selective arterial and venous vessel enhancement. The reviewer used maximum intensity projections (MIPs) and multi-planar reformations (MPRs) and had the availability over all source images. Analysis included assessment of aortic arch, brachial-cephalic artery, subclavian artery, axillary artery, brachial artery, radial artery, ulnar artery, superior vena cava, brachial-cephalic vein, subclavian vein, axillary vein, basilic vein and cephalic vein. Vessels were assessed for the presence of stenosis and occlusions. Furthermore, the location and caliber of cephalic vein side-branches (accessory veins) were determined. The percentage of accessory vein caliber in relation to cephalic vein caliber was calculated (accessory vein diameter / cephalic vein diameter * $100 \%$ ).

\section{Surgery}

All RC-AVF operations were performed under regional anesthesia by a single vascular surgeon with an extensive experience in vascular access surgery. An incision of $3 \mathrm{~cm}$ length was made approximately $5 \mathrm{~cm}$ proximal to the distal radial head. Both radial artery and cephalic vein were exposed. The cephalic vein was mobilized to approximate the radial artery after distal cephalic vein ligation. An anastomosis of $10 \mathrm{~mm}$ in length was created in a side-to-end fashion (radial artery to cephalic vein). Intra-operative assessment of cephalic vein thrill and flow-velocities by palpation and Doppler analysis were used to determine the instant RC-AVF function. 


\section{Postoperative assessment of RC-AVF function}

At 6 weeks post-operative, clinical RC-AVF function was assessed at the hemodialysis department. Maturation was defined as the ability to use the RCAVF for two-needle hemodialysis, judged by a nephrologist, vascular surgeon and dialysis access nurse. Clinically, non-maturation was defined as a RC-AVF being not functional within 6 to 12 weeks after creation.

\section{Salvage procedures}

Procedures such as percutaneous transluminal angioplasty (PTA), surgical creation of a more proximal anastomosis or ligation of accessory veins were performed in cases of non-maturation, in an attempt to salve the access early failure or non-maturation.

\section{Statistical analysis}

All statistical analysis was performed using SPSS 11.0.1 (SPSS Inc, Chicago, IL) for Windows (Microsoft, Redmond, WA). A stepwise forward logistic regression analysis was used to determine the statistical significance of preoperative parameters (age, gender, co-morbidities, radial artery diameter, radial artery flow, cephalic vein diameter, number of accessory veins and accessory vein caliber as percentage of cephalic vein caliber) for prediction of RC-AVF nonmaturation. $P$ values $<0.05$ were considered statistically significant. The sensitivity, specificity, positive predictive value (PPV) and negative predictive value (NPV) of preoperatively detected accessory veins and accessory vein caliber were calculated using the clinical outcome 6 weeks postoperative as standard of reference.

\section{Results}

A total of 15 RC-AVFs were created in 15 patients. Assessment of RC-AVF function 6 weeks postoperative revealed only 5 cases of successful maturation (group A) and 10 cases of access early failure $(n=1)$ non-maturation $(n=9)$ (group B). Patient characteristics were comparable for patients in both groups and are summarized in table1. 


$\begin{array}{lll} & \begin{array}{l}\text { Group } \boldsymbol{A} \\ \text { Maturation }\end{array} & \begin{array}{l}\text { Group B } \\ \text { Non-maturation }\end{array} \\ \text { Age } & 66 \pm 12.1 & 67 \pm 15.4 \\ \text { Males } & 60 \%(3 / 5) & 90 \%(9 / 10) \\ \text { BMI } & 27.5 \pm 5.6 & 25.5 \pm 3.7 \\ \text { PAOD } & 80 \%(4 / 5) & 40 \%(4 / 10) \\ \text { Diabetes } & 0 \%(0 / 5) & 20 \%(2 / 10) \\ \text { Hypertension } & 60 \%(3 / 5) & 80 \%(8 / 10)\end{array}$

Table 1: Patient characteristics. Values represent \pm means standard deviations, $\mathrm{BMI}=$ body mass index: $\mathrm{kg} / \mathrm{m} 2, \mathrm{PAOD}=$ peripheral arterial occlusive disease)

$\begin{array}{lll} & \begin{array}{l}\text { Group } \boldsymbol{A} \\ \text { Maturation }\end{array} & \begin{array}{l}\text { Group B } \\ \text { Non-maturation }\end{array} \\ \text { Radial artery diameter }(\mathrm{mm}) & 2.8 \pm 0.5 & 2.9 \pm 0.5 \\ \text { Radial artery flow }(\mathrm{mL} / \mathrm{min}) & 50.3 \pm 35.0 & 54.5 \pm 42.9 \\ \text { Cephalic vein diameter }(\mathrm{mm}) & 2.6 \pm 0.8 & 2.9 \pm 0.7 \\ \text { Number of accessory veins } & 2.0 \pm 0.7 & 2.8 \pm 1.5 \\ \text { Accessory vein caliber } & 66.6 \% \pm 10.5 \% & 88.9 \% \pm 8.9 \%^{*}\end{array}$

Table 2: Preoperative DUS results, (Values represent \pm means standard deviations, * $P=0.01$ )

Preoperative diameter and flow measurement results were also comparable between both groups (table 2). Accessory veins were present in all cases and the number of accessory veins did not differ between group $A$ and $B$. Accessory vein caliber (as percentage of cephalic vein diameter) was the only parameter with significant predictive value for RC-AVF non-maturation as determined by the stepwise forward logistic regression analysis $(P=0.01)$. The caliber of accessory veins in group A was smaller compared to group B. Preoperative detected accessory veins with a diameter $>70 \%$ of the cephalic vein diameter, had a sensitivity, specificity, PPV and NPV of $80 \%, 100 \%, 100 \%$ and $91 \%$, respectively. For a cut-off value of $75 \%$ the sensitivity, specificity, PPV and NPV were $100 \%$, 90\%, 83\% and 100\%, respectively. Preoperative CE-MRA images of two patients with a failed RC-AVF due to large caliber accessory veins are shown in figure 1 (left and centre). In one patient the RC-AVF matured successfully despite the presence of a large caliber accessory vein (79\% of the cephalic vein diameter) (figure 1 right). 

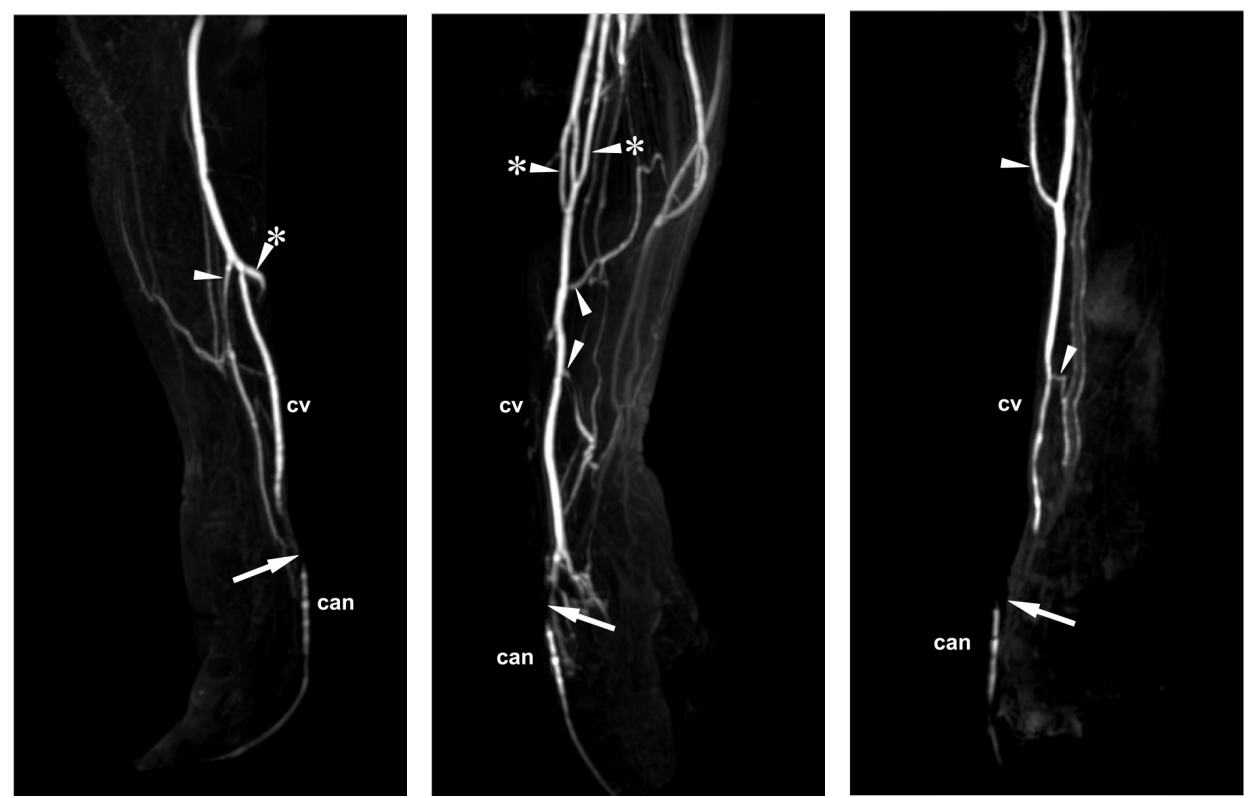

Figure 1: Maximum intensity projection of preoperative forearm CE-MR venograms in patients with RC-AVF non-maturation (left and centre) and a patient in with a RC-AVF that matured successfully (right). The arrowheads point at accessory veins. Surgical ligation of accessory veins as indicated by an arrowhead with a star, resulted in successful salvage of RC-AVF non-maturation. The arrow points at the intravenous cannula (can) that resulted in $\mathrm{mr}$-signal extinction.

Salvage procedures were performed in 9 out 10 cases of access non-maturation with an overall success rate of $89 \%$ (8/9). One with RC-AVF non-maturation received a renal transplant before an attempt of salvage was initiated. In four patients, accessory vein ligation alone was a successful salvage procedure. Dilatation of a cephalic vein stenosis was successful in two patients. In another two patients, dilatation of a cephalic vein stenosis alone was unsuccessful and accessory vein ligation turned out to be a successful additional salvage procedure in these two cases. In one patient in whom the RC-AVF thrombosed within two weeks, dilatation of multiple cephalic vein stenosis and ligation of accessory veins did not result in successful salvage and a new vascular access was created. A total of 13 out of 15 (87\%) RC-AVFs were functional after salvage procedures. 


\section{Discussion}

This is the first report on the value of preoperative accessory vein assessment for prediction of RC-AVF early failure and non-maturation. Large caliber accessory veins are associated with RC-AVF early failure and non-maturation as evidenced by the successful maturation after accessory vein ligation as a single procedure in a substantial group of cases.

Early failure and non-maturation of a newly created hemodialysis vascular access may necessitate the use of central venous catheters. This superimposes these patients to all risks involved in central venous catheter use, such as; infection, central venous obstruction and increased risk of future access failure (4). Since it is likely that a patient requiring long-term hemodialysis vascular access will require multiple vascular accesses in the future, early failure and non-maturation of newly created AVFs and central venous catheter use should be prevented.

The exact mechanism of maturation and non-maturation remain largely unclear as evidenced by the reported persisting high non-maturation rates, up to $50 \%(2,3)$. The presence of a large caliber accessory vein is disadvantageous for a RC-AVF maturation because it may lead to a decrease in cephalic vein flow and decrease in cephalic vein diameter due to a decrease in intravenous pressure $(10,14)$.

Beathard et al., reported a group of 100 patients with failed AVFs (5). Venous stenosis, accessory veins or both were present in $78 \%, 46 \%$ and $24 \%$ of the cases, respectively. Accessory vein ligation as a single salvage procedure had a success-rate of $100 \%$ in the group patients with accessory veins (5). Our findings are in agreement with these results. In the current study we found venous stenosis and large caliber accessory veins to be the cause of non-maturation in all cases. No venous stenosis was detected preoperatively by either DUS or CEMRA. It seems therefore plausible that stenosis developed after RC-AVF creation and non-maturation due to venous stenosis could therefore not have been anticipated.

The additive value of the findings of the current study, in addition to the results of Beathard et al., is that preoperatively detected large caliber accessory veins, as detected by CE-MRA, are associated with RC-AVF non-maturation. Ligation of 
these large caliber accessory veins was a successful salvage procedure in these patients. We believe that non-maturation of RC-AVF due to large caliber accessory veins can therefore potentially be anticipated by ligation of these accessory veins during initial RC-AVF creation.

In the current study, CE-MRA was used for localization and diameter measurements of accessory veins. For clinical practice however, DUS is a more attractive modality for this purpose because it is; less expensive, non-invasive, not associated with the use of contrast-media and is readily available.

The current study has limitations. The sample size was limited in this study. Furthermore, no postoperative venous flow measurements by duplex ultrasound have been performed to determine the effect of accessory vein diameter on cephalic vein flow and cephalic vein diameter. Because cephalic vein diameter and flow have been reported to be important parameters that enable identification of RC-AVF maturation based on ultrasonographic criteria this would have add to the value of the results of the current study (9). Unfortunately we did not determine the distribution of the arteriovenous anastomotic flow over the cephalic vein and accessory veins, which potentially would have shown the hemodynamic effects of large caliber accessory veins.

In conclusion: preoperatively present large caliber accessory veins are associated with RC-AVF non-maturation. Ligation of large caliber accessory veins is a successful salvage procedure in a substantial group of patients. Future studies need to determine if ligation of large caliber accessory veins during initial RCAVF creation will reduce non-maturation rates. 


\section{References}

1. Clinical practice guidelines for vascular access. Am J Kidney Dis 2006;48 Suppl 1:S176-247.

2. Allon M, Lockhart ME, Lilly RZ, Gallichio MH, Young CJ, Barker J, et al. Effect of preoperative sonographic mapping on vascular access outcomes in hemodialysis patients. Kidney Int 2001;60(5):2013-20.

3. Miller PE, Tolwani A, Luscy CP, Deierhoi MH, Bailey R, Redden DT, et al. Predictors of adequacy of arteriovenous fistulas in hemodialysis patients. Kidney Int 1999;56(1):275-80.

4. Rayner HC, Pisoni RL, Bommer J, Canaud B, Hecking E, Locatelli F, et al. Mortality and hospitalization in haemodialysis patients in five European countries: results from the Dialysis Outcomes and Practice Patterns Study (DOPPS). Nephrol Dial Transplant 2004;19(1):108-20.

5. Beathard GA, Arnold P, Jackson J, Litchfield T. Aggressive treatment of early fistula failure. Kidney Int 2003;64(4):1487-94.

6. Beathard GA, Settle SM, Shields MW. Salvage of the nonfunctioning arteriovenous fistula. Am J Kidney Dis 1999;33(5):910-6.

7. Wong V, Ward R, Taylor J, Selvakumar S, How TV, Bakran A. Factors associated with early failure of arteriovenous fistulae for haemodialysis access. Eur $\mathrm{J}$ Vasc Endovasc Surg 1996;12(2):207-13.

8. Turmel-Rodrigues L, Mouton A, Birmele B, Billaux L, Ammar N, Grezard O, et al. Salvage of immature forearm fistulas for haemodialysis by interventional radiology. Nephrol Dial Transplant 2001;16(12):2365-71.

9. Robbin ML, Chamberlain NE, Lockhart ME, Gallichio MH, Young CJ, Deierhoi MH, et al. Hemodialysis arteriovenous fistula maturity: US evaluation. Radiology 2002;225(1):59-64.

10. Planken RN, Huberts W, Bosboom EMH, Vosse van der FN, Leiner T, Tordoir JHM. The impact of accessory veins on arteriovenous fistula flow distribution as determined by mathematical flow modeling. In: Henry $\mathrm{MH}$, editor. Vascular Access for Hemodialysis - X. Chigaco, IL: Gore; 2006.

11. Tordoir $\mathrm{JH}$, Mickley V. European guidelines for vascular access: clinical algorithms on vascular access for haemodialysis. Edtna Erca J 2003;29(3):131-6.

12. Planken RN, Tordoir JHM, de Haan MW, Backes WH, van Engelshoven JMA, Leiner T. Selective MR-Arteriography and Venography Compared to Ultrasonography of the Entire Upper Extremity prior to Arteriovenous Fistula Placement for Hemodialysis. In: Radiological Society of Northern America scientific assembly and annual meeting program; 2005; Oak Brook, IL; 2005. p. 407.

13. van Erkel AR, Pattynama PM. Receiver operating characteristic (ROC) analysis: basic principles and applications in radiology. Eur J Radiol 1998;27(2):88-94.

14. Planken RN, Keuter XH, Kessels AG, Hoeks AP, Leiner T, Tordoir JH. Forearm cephalic vein cross-sectional area changes at incremental congestion pressures: towards a standardized and reproducible vein mapping protocol. J Vasc Surg 2006;44(2):353-8. 




\section{Chapter 8}

Stenosis detection in forearm hemodialysis arteriovenous fistulae by multiphase contrast- enhanced magnetic resonance angiography: preliminary experience

R.N. Planken, J.H.M. Tordoir, R. Dammers, M.W. de Haan, T.K. Oei, F.M. van der Sande, J.M.A. van Engelshoven, T. Leiner

Journal of Magnetic Resonance Imaging 2003: 17(1) 54-64 


\section{Abstract}

Purpose: To assess the feasibility and accuracy of multiphase contrast-enhanced magnetic resonance angiography (CE-MRA) in patients with dysfunctioning hemodialysis arteriovenous fistulae (AVF), using digital subtraction angiography (DSA) as the standard of reference.

Materials and methods: Fifteen patients with dysfunctioning AVF (eight radiocephalic and seven graft AVF) underwent CE-MRA. Dysfunction was defined as a flow decline of more than $25 \%$ in 1 month measured by dilutional flow measurements. CE-MRA was performed during injection of $35 \mathrm{~mL}$ of gadolinium-DTPA. The CE-MRA sequence consisted of a time-resolved series of 10 scans, each lasting approximately 10 seconds. The technical parameters were $\mathrm{TR} / \mathrm{TE} / \mathrm{FA} /$ voxel $=5.4 / 1.6 / 40 / 3.1 \mathrm{~mm} 3$, and a rectangular surface reception coil was used. All patients were scheduled to undergo DSA at which an intervention was carried out when a stenosis $>50 \%$ was seen. Two observers, unaware of each other's findings and the findings at DSA, quantified the number and degree of stenosis in the failing AVF. Image quality for CE-MRA and DSA was scored on a 3-point scale. The diagnostic performance of CE-MRA was analyzed with receiver-operator characteristic $(\mathrm{ROC})$ analysis.

Results: CE-MRA and DSA examinations were performed without side effects in all 15 patients. Image quality was scored significantly better on CE-MRA (observer 1: CE-MRA, 2.0; DSA, 1.3; $\mathrm{P}=.001$; observer 2: CE-MRA, 2.0; DSA, 1.4; $P=.002)$. Interobserver agreement for detection of $>50 \%$ stenosis was 0.81 (95\% confidence interval $(\mathrm{Cl})=0.71-0.92)$ for CE-MRA and $0.69(95 \% \mathrm{Cl}$ $=0.55-0.84$ ) for DSA. ROC analysis revealed a mean area under the curve of 0.78 . On the patient level, at the $>50 \%$ threshold, mean sensitivity, specificity, positive predictive value (PPV), and negative predictive value (NPV) were $100 \%$ (95\% Cl =69\%-100\%), 10\% (95\% Cl =0\%-78\%), 70\% (95\% Cl = 38\%-92\%), and $100 \%(95 \% \mathrm{Cl}=50 \%-100 \%)$, respectively. At the $>75 \%$ threshold, mean sensitivity, specificity, PPV, and NPV were 75\% (95\% Cl $=20 \%-99 \%), 78 \%$ (95\% Cl $=39 \%-98 \%), 55 \%(95 \% \mathrm{Cl}=12 \%-96 \%)$, and $89 \%(95 \% \mathrm{Cl}=52 \%-$ $100 \%)$, respectively.

Conclusion: CE-MRA is a useful diagnostic tool for detecting stenoses in flowdeclined hemodialysis AVF prior to interventional DSA. 


\section{Introduction}

Arteriovenous fistulae (AVF) for hemodialysis vascular access have a high risk of thrombosis due to stenoses that jeopardize flow and patency (1). Early detection and treatment of these stenoses can prevent thrombosis (2). In current clinical practice, stenosis detection and grading in AVF and the venous outflow segments are performed using digital subtraction angiography (DSA). The arterial part of the $A V F$ is visualized according to the method of Staple, using a proximal cuff to interrupt flow in order to achieve retrograde filling of the arterial part of the AVF (3). Analysis of DSA images acquired with this method can be difficult due to vessel overlap, especially at the level of the anastomoses. Due to the temporary flow interruption, the hemodynamic situation is altered, which potentially limits the value of DSA. Furthermore, because of incomplete retrograde filling, the feeding artery and arterial part of the AVF are not always depicted in their entirety $(4,5)$.

Another way of diagnosing the extent and severity of AVF stenoses is magnetic resonance angiography (MRA), which is not accompanied by radiation exposure and offers the ability to acquire three-dimensional (3D) data sets, which may potentially solve the problem of vessel overlap. Varying results have been published concerning the ability of MRA to depict and detect flowlimiting stenoses in AVF using time-of-flight (TOF) (5-9), phase contrast (PC), and contrast-enhanced (CE) MRA (5) techniques. In these studies, the use of TOF and PC-MRA resulted in stenosis overestimation because of flow voids caused by poststenotic intravoxel phase dispersion (5-9). With CE-MRA, better results have been obtained, but experience is limited (5).

The aim of this prospective study was to assess the accuracy of high-resolution multiphase 3D CE-MRA for detection and grading of stenoses in dysfunctioning $A V F$ in comparison to DSA, which was used as the standard of reference.

\section{Materials and methods}

\section{Patients}

Based on the Dialysis Outcomes Quality Initiative (DOQI) guidelines (2), monthly flow measurements were performed in every hemodialysis patient at the Department of Nephrology in our hospital using an ultrasound dilution technique 
(Transonic ${ }^{\circledR}$ Systems Inc., Ithaca, NY) (2). If absolute AVF flow at any time was $<600 \mathrm{~mL} /$ minute or if a patient exhibited a flow decline of $25 \%$ between two consecutive measurements in combination with an absolute flow of $<1000$ $\mathrm{mL} /$ minute, the fistula was considered at risk for thrombosis (2). Subsequently, these patients were referred for DSA and asked to participate in the study. A total of 15 consecutive hemodialysis patients ( 6 males, 9 females; mean age = 67 years; range $=60-79$ ) with failing AVF were included.

All patients had a forearm AVF (eight radiocephalic and seven graft). The mean number of previous failed AVF was 1.4 per patient (range $=1-3$ ), with a mean of 1.3 (range $=1-2$ ) AVF in the ipsilateral arm. The AVF were in position for 2-60 months (mean $=17.6$ ), with a mean intervention rate in the current AVF of 1.6 (range $=0-5$ ) per patient per year (radiocephalic $=0.8$, graft $=2.2$ ). The institutional review board had approved the protocol, and all patients signed informed consent before they were enrolled in the study.

\section{Multiphase contrast-enhanced magnetic resonance angiography}

For all acquisitions, we used a commercially available 1.5-T MR scanner (Intera, release 8.1; Philips Medical Systems, Best, The Netherlands) with a gradient strength of $23 \mathrm{mT} / \mathrm{m}$ and a slew rate of $115 \mathrm{mT} / \mathrm{m} / \mathrm{msec}$. For signal transmission, the built-in quadrature body coil was used; for signal reception, a rectangular $(400 \times 100 \mathrm{~mm})$ surface coil (Philips Medical Systems, Best, The Netherlands) was used in all acquisitions. Prior to imaging, venous access was established in an antecubital vein of the upper extremity contralateral to the AVF using an 18-gauge intravenous cannula (Venflon; Ohmeda, Helsingborg, Sweden). Patients were placed on the scanner table in the prone position with the forearm with the AVF elevated above their head and entered the scanner headfirst. A TOF scan was obtained of the forearm in order to localize the AVF and its afferent and efferent vessels. A gradient-recalled echo (GRE) (turbo field echo (TFE) pulse sequence with a flip angle sweep and a $180^{\circ}$ inversion prepulse for optimal background tissue suppression was used. Parameters for the TOF sequence were $\mathrm{TR} / \mathrm{TE}=12 / 2.5 \mathrm{msec}$, flip angle $=50^{\circ}$, field of view (FOV) $=$ $430 \times 300 \mathrm{~mm}$, matrix $\times 256 \times 128,60$ axial slices with 4.0-mm slice thickness, 3.0-mm interslice gap, and a concatenated saturation band superior to the imaged slice. Total scan time was 129 seconds. From the thus acquired TOF data 
set, orthogonal maximum intensity projection (MIP) images were generated and used to prescribe the 3D CE-MRA scans, covering the entire forearm with the AVF, including the cubital fossa. The 3D CE-MRA scan was planned so that all arteries as seen on the orthogonal MIP images obtained with the TOF scans were included in the volume. In addition, TOF source images were reviewed to make sure no arteries were incorrectly excluded from the $3 D$ scan due to in-plane saturation effects. A 3D GRE pulse sequence with partial echo sampling was used with the following imaging parameters: TR/TE $=5.1 / 1.5 \mathrm{msec}$, flip angle $=$ $40^{\circ}, \mathrm{FOV}=300 \times 90 \mathrm{~mm}$, matrix $=304 \times 58$, and 30-37 coronal partitions with 2.0-mm slice thickness. The acquisition voxel size was $3.1 \mathrm{~mm}^{3}$; after zero filling in the slice direction, the interpolated voxel size was $1.5 \mathrm{~mm}^{3}$. The $\mathrm{K}$-space filling was set to elliptical centric (CE timing robust angiography (CENTRA) per Philips terminology). In all patients 10 immediately consecutive 3D CE-MRA scans were acquired. Scan time was dependent on the exact number of partitions and varied between 92 and 110 seconds. At exactly the same time the 3D CE-MRA commenced, injection of contrast medium was started $(35 \mathrm{~mL}$ of gadoliniumDTPA; Magnevist, Schering, Berlin, Germany), followed by injection of $20 \mathrm{~mL}$ of saline using an MR-compatible remote-controlled power injector (Medrad Spectris; Medrad, Indianola, PA). The injection rate for both contrast medium and saline was set to $3.0 \mathrm{~mL} /$ second. Since a multiphase technique was used, image acquisition was started together with injection of contrast medium so that no additional timing of contrast medium arrival in the AVF was needed. To increase vessel-to-background contrast, the first (nonenhanced) $3 \mathrm{D}$ volume was later subtracted on partition basis from the subsequent identical scans obtained during the presence of contrast material. In all cases, CE-MRA was performed prior to DSA.

\section{Digital subtraction angiography}

All DSA examinations were performed by experienced interventional radiologists on Philips Integris V5000 (Philips Medical Systems, Best, The Netherlands) digital angiography equipment. Selective arteriograms were obtained by puncturing the AVF retrogradely with 19-gauge needle and subsequently advancing a 4-French ( $F r$ ) end-hole catheter (Cordis, Miami, FL) into the venous part of the AVF. Images of the feeding artery, anastomosis (in the case of radiocephalic $A V F$ ), and the arterial part of the AVF were obtained by retrograde 


\section{Chapter 8}

filling of the AVF with contrast material due to flow interruption, according to Staple (3). The total amount of contrast medium that was used varied between 60 and $80 \mathrm{~mL}$ of lohexol (Omnipaque; Nycomed-Amersham, Oslo, Norway) and was administered in a variable number of runs. Contrast medium was administered with a power injector (Medrad, Indianola, PA). The number of contrast injections and view angles varied per patient and depended on the complexity of the vascular anatomy. If a stenosis $>50 \%$ luminal reduction was found on DSA images, it was dilated. If no apparent stenosis was found at DSA, CE-MRA images were made available and compared with DSA images. Any lesions found on CE-MRA and not visualized at DSA were then, if possible, depicted with DSA and, when deemed necessary, dilated.

\section{Image analysis}

For image analysis purposes, all CE-MRA data sets were transferred to an offline workstation (Ultrasparc 30; Sun Microsystems, Sunnyvale, CA), with dedicated postprocessing software (EasyVision 4.0; Philips Medical Systems, Best, The Netherlands). Review was done on the dynamic series, which exhibited the best selective AVF enhancement. The reviewers used MIPs and multiplanar reformations (MPRs) and had the availability over source images when necessary. Stenosis measurements were carried out using an electronic caliper with an accuracy of $0.1 \mathrm{~mm}$. DSA images were filmed and analyzed on the hard copies with a loupe and caliper.

CE-MRA and DSA images were read in random order, in a prospective, blinded manner by two radiologists experienced in vascular imaging. Between the two reads of the same patient, a period of at least 2 weeks elapsed. Both radiologists were not involved in either the DSA or CE-MRA procedures and were unaware of each other's results and of results obtained with any other imaging modality (e.g., duplex ultrasonography). Upper extremity vessels and AVF were subdivided into segments for analysis, which are shown in Figure 1. 


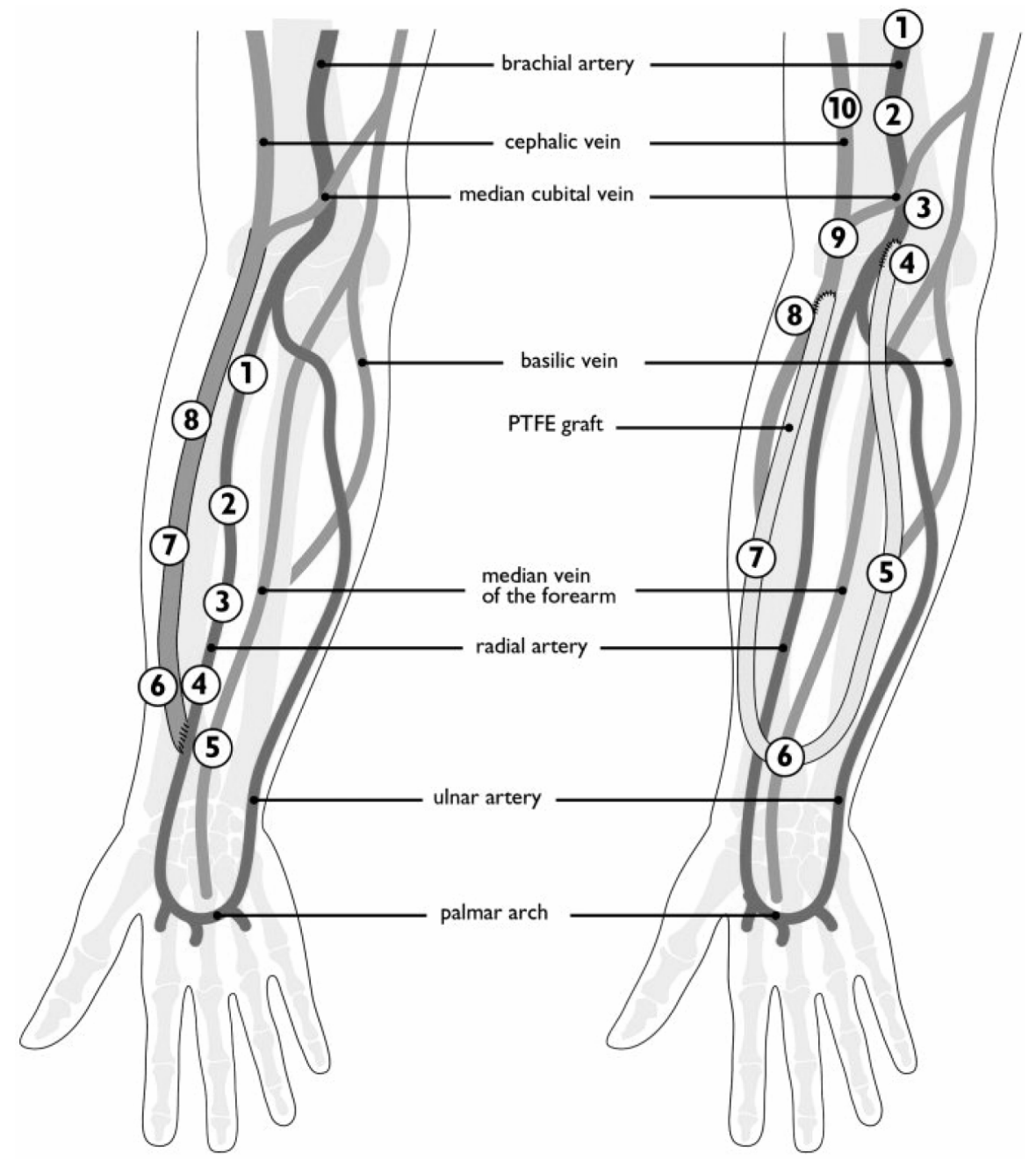

1 afferent artery $>10 \mathrm{~cm}$ proximal from anastomosis 2 afferent artery $5-10 \mathrm{~cm}$ proximal from anastomosis 3 afferent artery $2-5 \mathrm{~cm}$ proximal from anastomosis

4 afferent artery $<2 \mathrm{~cm}$ proximal from anastomosis 5 anastomosis

6 efferent vein $<2 \mathrm{~cm}$ proximal from anastomosis

7 efferent vein $2-5 \mathrm{~cm}$ proximal from anastomosis

8 efferent vein $>5 \mathrm{~cm}$ proximal from anastomosis

1 afferent artery $>5 \mathrm{~cm}$ proximal from arterial anastomosis

2 afferent artery $2-5 \mathrm{~cm}$ proximal from arterial anastomosis

3 afferent artery $<2 \mathrm{~cm}$ proximal from arterial anastomosis

4 arterial anastomosis

5 arterial part of the PTFE loop graft

6 middle part of the PTFE loop graft

7 venous part of the PTFE loop graft

8 venous anastomosis

9 efferent vein $<2 \mathrm{~cm}$ proximal from venous anastomosis

10 efferent vein $<5 \mathrm{~cm}$ proximal from venous anastomosis

Figure 1: Schematic overview of both AVF types with the different segmental subdivisions. On the left, the radiocephalic AVF is shown (divided into 8 segments), and on the right, the polytetraflourethylene (PTFE) loop graft AVF is shown (divided into 10 segments). 


\section{Chapter 8}

In every segment, all visible vessel diameter reductions were measured. Stenoses were measured by dividing the luminal diameter that exhibited the highest grade of stenosis by the luminal diameter of the closest adjacent normal part of the vessel and classified on a 5 -point scale $(1=0 \%-20 \%, 2=21 \%-49 \%, 3=50 \%$ $74 \%, 4=75 \%-99 \%, 5=$ occlusion). Diameter reductions $>50 \%$ were considered hemodynamically significant (2). For analysis purposes, only the most severe stenosis per vessel segment was taken into account. In addition to number and grade of stenoses, the evaluated CE-MRA and DSA images were also judged with respect to image quality on a 3 -point scale $(0=$ nondiagnostic, $1=$ mediocre diagnostic, 2 = excellent diagnostic).

\section{Statistical analysis}

Sensitivity, specificity, positive predictive value (PPV), and negative predictive value (NPV) were calculated for each observer using DSA as the standard of reference.

The diagnostic properties of CE-MRA were evaluated using ROC analysis, in which the sensitivity of CE-MRA was plotted against the complement of specificity for different grades of stenosis. To assess overall accuracy of CE-MRA compared to the standard of reference, areas under the curve were calculated for both observers (10). With the Wilcoxon signed ranks test (SPSS, version 10.0.5; SPSS Inc., Chicago, IL), any differences between CE-MRA and DSA in image quality were analyzed on patient level. To determine interobserver agreement with regard to stenosis detection between the two radiologists, the linear weighted kappa statistic was calculated for both DSA and CE-MRA (11). P values less than 0.05 were considered significant.

\section{Results}

\section{Imaging procedures}

All 15 patients underwent CE-MRA and DSA (radiocephalic $=8$, loop graft $=7$ ) successfully. In 13/15 patients, the apparent cause of the flow decline was found with DSA, and percutaneous transluminal angioplasty (PTA) treatment was successful in 10/13 patients, in terms of recovered flow as measured during the following dialysis sessions. In 2/15 patients, DSA was non-diagnostic and CEMRA images were analyzed for any additional lesions that were not apparent on 
DSA; those lesions were subsequently treated with PTA. A typical example for both modalities is shown in Figure 2.
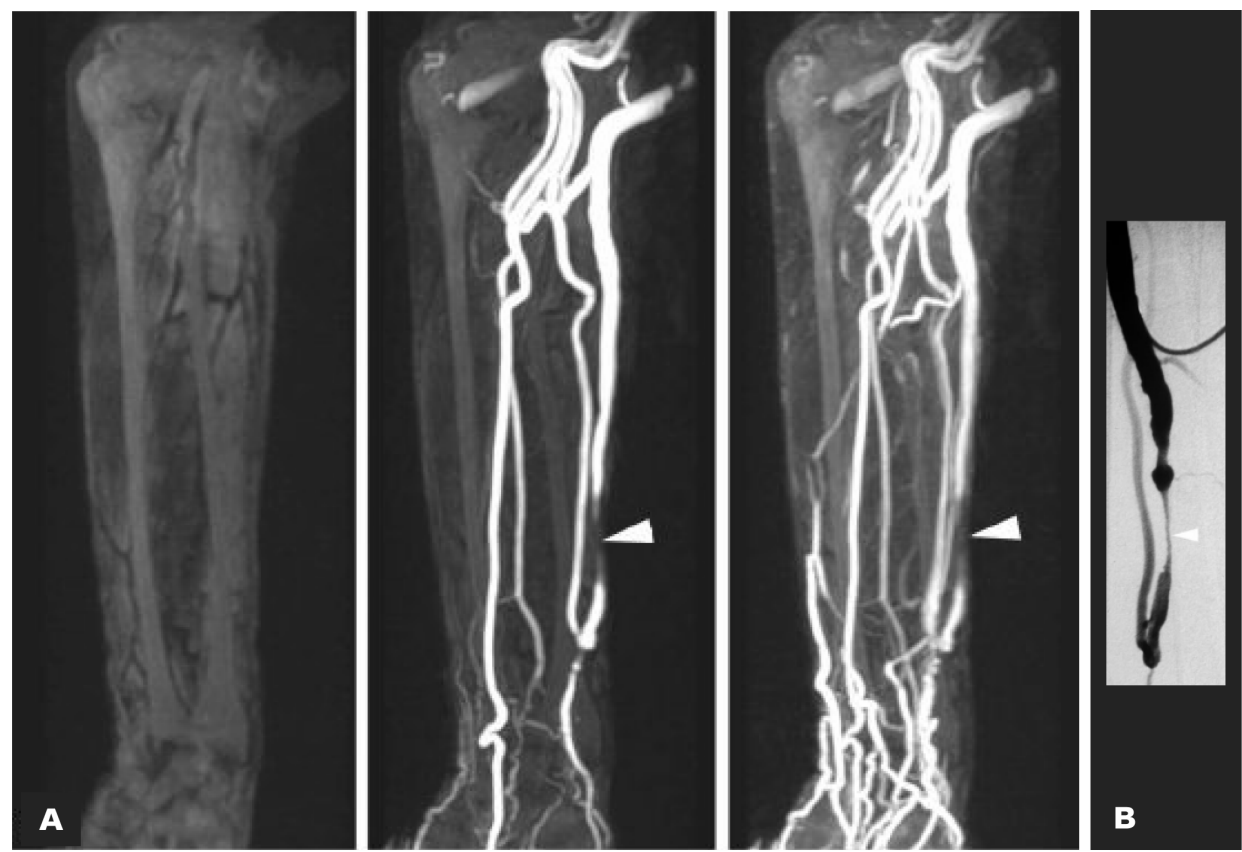

Figure 2: (A) Whole-volume MIPs of three sequential phases of a multiphase CE-MR angiogram of a radiocephalic AVF obtained in the left arm of a 61 -year-old male patient. The left panel (acquisition started together with administration of contrast material) shows a native MIP before the arrival of contrast material in the forearm; the middle panel shows the first arterial phase (10.3 seconds after the first phase) with a clearly visible highgrade stenosis in the cephalic vein draining the fistula (arrowhead). In the right panel, the second arterial phase (20.6 seconds after the first phase) is shown in which distal arm and hand veins are also visible in addition to the stenosis. (B) A detail is shown of the highgrade stenosis in a corresponding DSA image (right panel,).

\section{Stenosis detection}

In 15 patients there were potentially 134 vessel segments available for analysis with CE-MRA. All 134 vessel segments were considered diagnostic by both observers. Observer 1 considered 90 vessel segments evaluable, and observer 2 considered 98 vessel segments evaluable on DSA. Less segments could be evaluated with DSA because they were not depicted (both observers: $n=33$ ) or visible segments were considered of nondiagnostic image quality (observer $1: n=$ 11; observer 2: $n=3$ ). ROC analysis of both observers' interpretations yielded areas under the curve of 0.74 for observer 1 and 0.81 for observer 2 (Fig. 3). On patient level, sensitivity and specificity for identifying $>50 \%$ stenosis were 100\% 
(95\% confidence interval $(\mathrm{Cl})=69 \%-100 \%)$ and $0 \%(95 \% \mathrm{Cl}=0 \%-48 \%$ ), respectively, for observer 1 ; for observer 2 , these values were $100 \%(95 \% \mathrm{Cl}=$ $69 \%-100 \%)$ and $20 \%(95 \% \mathrm{Cl}=1 \%-71 \%)$. PPV was $69 \%(95 \% \mathrm{Cl}=38 \%-88 \%)$ and NPV was $100 \%(95 \% \mathrm{Cl}=50 \%-100 \%)$ for observer 1 ; for observer 2, PPV was $71 \%(95 \% \mathrm{Cl}=42 \%-92 \%)$ and NPV was $100 \%(95 \% \mathrm{Cl}=50 \%-100 \%)$. At the $>75 \%$ threshold level, sensitivity, specificity, PPV, and NPV were $75 \%(95 \%$ $\mathrm{Cl}=20 \%-99 \%), 73 \%(95 \% \mathrm{Cl}=39 \%-94 \%), 50 \%$ (95\% Cl = 12\%-88\%), and 89\% (95\% Cl $=52 \%-100 \%$ ), respectively, for observer 1 ; for observer 2, the corresponding values were $75 \%(95 \% \mathrm{Cl}=20 \%-99 \%), 82 \%(95 \% \mathrm{Cl}=48 \%$ $98 \%$ ), $60 \%$ (95\% Cl $=15 \%-96 \%)$, and $90 \%$ (95\% Cl = 56\%-100\%). Table 1 lists the numbers of true positive, true negative, false positive, and false negative vessel segments for both observers.

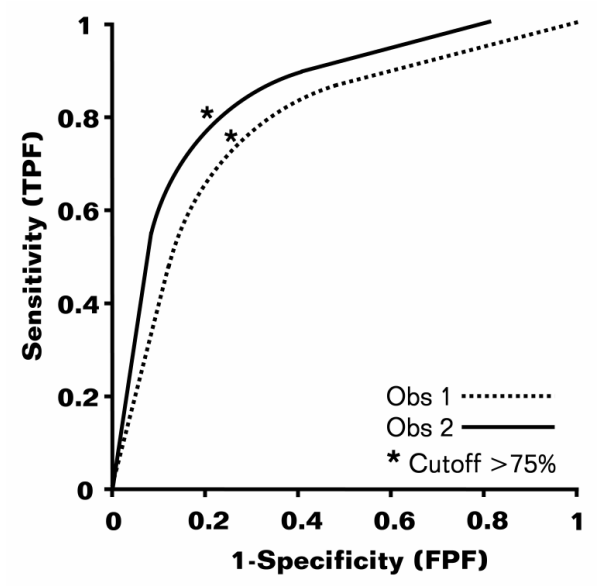

Figure 3: Obs, observer; TPF, true positive fraction; FPF, false positive fraction. 


\begin{tabular}{|c|c|c|c|c|c|c|c|c|c|c|c|c|c|}
\hline \multicolumn{9}{|c|}{$\begin{array}{c}\text { Observer } 1 \\
D S A\end{array}$} & \multicolumn{4}{|c|}{$\begin{array}{c}\text { Observer } 2 \\
\text { DSA }\end{array}$} & \\
\hline \multicolumn{2}{|c|}{ Stenosis grade } & 1 & 2 & 3 & 4 & 5 & \multicolumn{2}{|c|}{ Stenosis grade } & 1 & 2 & 3 & 4 & 5 \\
\hline & 1 & 0 & 0 & 0 & 0 & 0 & & 1 & 0 & 0 & 0 & 0 & 0 \\
\hline & 2 & 0 & 0 & 0 & 0 & 0 & & 2 & 1 & 0 & 0 & 0 & 0 \\
\hline \multirow[t]{3}{*}{$C E-M R A$} & 3 & 2 & 1 & 5 & 1 & 0 & CE-MRA & 3 & 1 & 3 & 4 & 1 & 0 \\
\hline & 4 & 0 & 2 & 1 & 3 & 0 & & 4 & 0 & 0 & 2 & 3 & 0 \\
\hline & 5 & 0 & 0 & 0 & 0 & 0 & & 5 & 0 & 0 & 0 & 0 & 0 \\
\hline
\end{tabular}

Table 1: Stenosis detection on CE-MRA compared to DSA for observer 1 and 2 (analysis was done on patient level; in every cell is the number of patients with the particular combination of test results on CE-MRA and DSA. See text for definition of stenosis categories).

On patient level, the discriminatory power for detection of stenoses was substantially better when $75 \%$ stenosis was used as a positive test result instead of $50 \%$. Both observers detected substantially more stenoses at CE-MRA than on the corresponding DSA images. Observer 1 detected 19 stenoses $\geq 50 \%$ on $\mathrm{CE}$ MRA and 11 on DSA. Observer 2 detected 22 stenoses on CEMRA and 11 on DSA. Reasons for the mismatch were overestimation on CE-MRA of borderline stenoses (observer 1: $n=2$; observer 2: $n=7$ ); false negative DSA (which became apparent during surgical shunt revision; $n=1$ ); false negative DSA due to dilation and filling of cephalic vein branches (an example of which is shown in Fig. 4), which, in retrospect, rendered vessel segments not interpretable (observer 1: $n=3$; observer 2: $n=2$ ); and false negative DSA due to incomplete filling of afferent arterial segments that were (erroneously) still considered interpretable (observer 1: $n=2$; observer $2: n=1$ ). In the vessel segments that were not depicted with DSA, observer 1 detected two additional stenoses on the CE-MRA images. In 3/13 patients, DSA was considered diagnostic, but initial PTA treatment was unsuccessful because access flow did not recover. In two of these three patients a lesion at the arterial anastomosis of the loop graft AVF was visible on CE-MRA, but missed on DSA because of incomplete depiction (Fig. 5). After both patients underwent PTA treatment of these lesions, flow recovered. In the third patient, kinking of the proximal radial artery was seen on CE-MRA and missed on DSA (Fig. 6). Surgical removal of the kink led to flow 


\section{Chapter 8}

recovery. All procedures with both modalities were performed without any complications.

Image quality

CE-MRA provided images of diagnostic image quality in 15/15 (100\%) patients, and DSA in 13/15 (86.7\%) patients (8 radiocephalic AVF, 7 graft AVF). CE-MRA image quality was scored significantly higher than that of DSA by both observers. The mean segment scores on patient level were as follows: for observer 1, CEMRA, 2.0; DSA, 1.3; $\mathrm{P}=0.001$; and for observer 2, CE-MRA, 2.0; DSA, 1.4; $\mathrm{P}=$ 0.002 . 

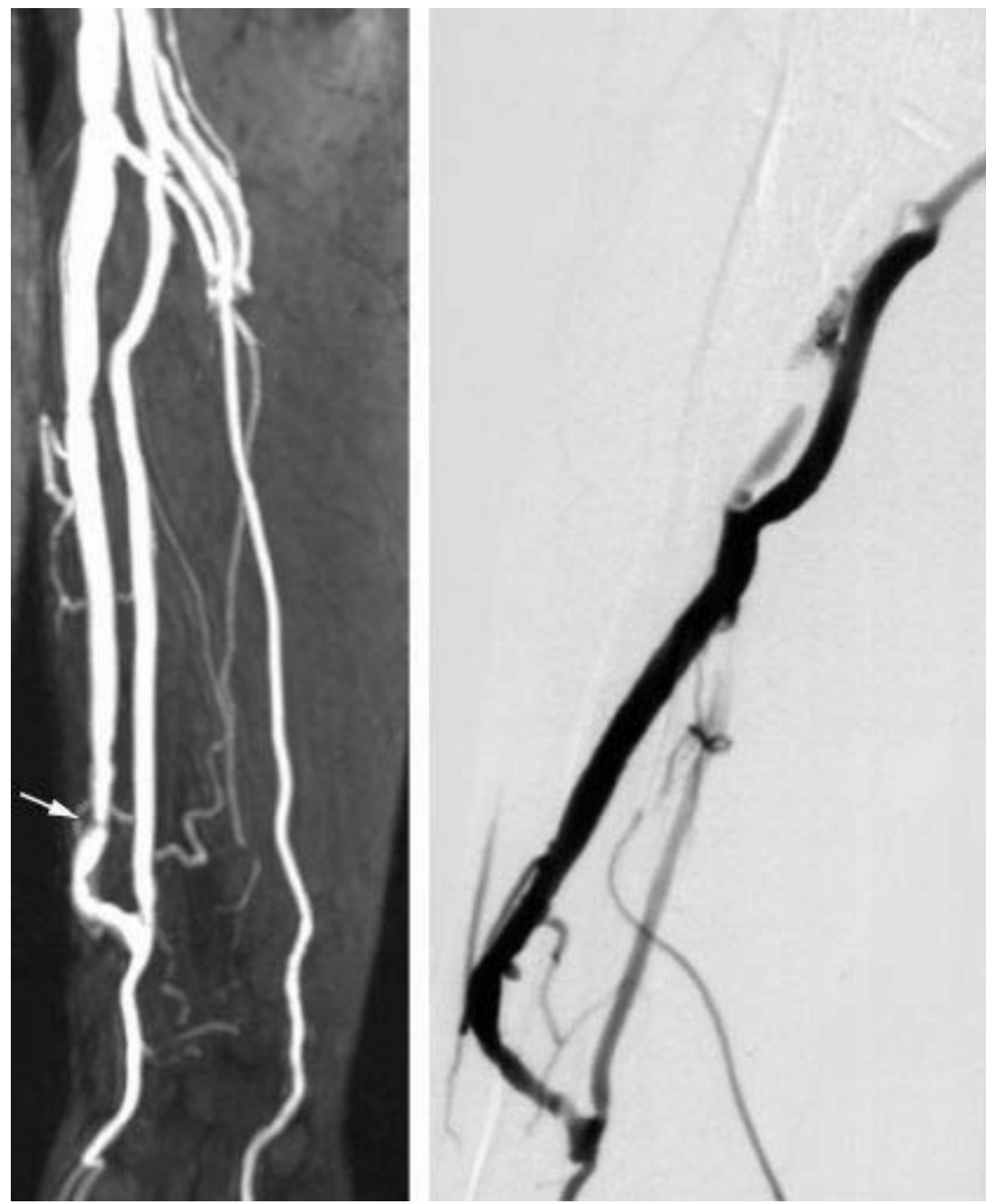

Figure 5: Whole-volume MIPs (left panel) of the second phase (10.1 seconds after start of injection of contrast medium) of a multiphase CE-MR angiogram of a radiocephalic AVF obtained in the left arm of a 69-year-old female patient. In the CE-MR angiogram, a stenosis $>50 \%$ is visible in the draining vein. This stenosis is not visible on the DSA, and as such, the CE-MRA is false positive. The reason that the DSA did not show the stenosis is because of dilation of veins due to the use of the Staple technique. 

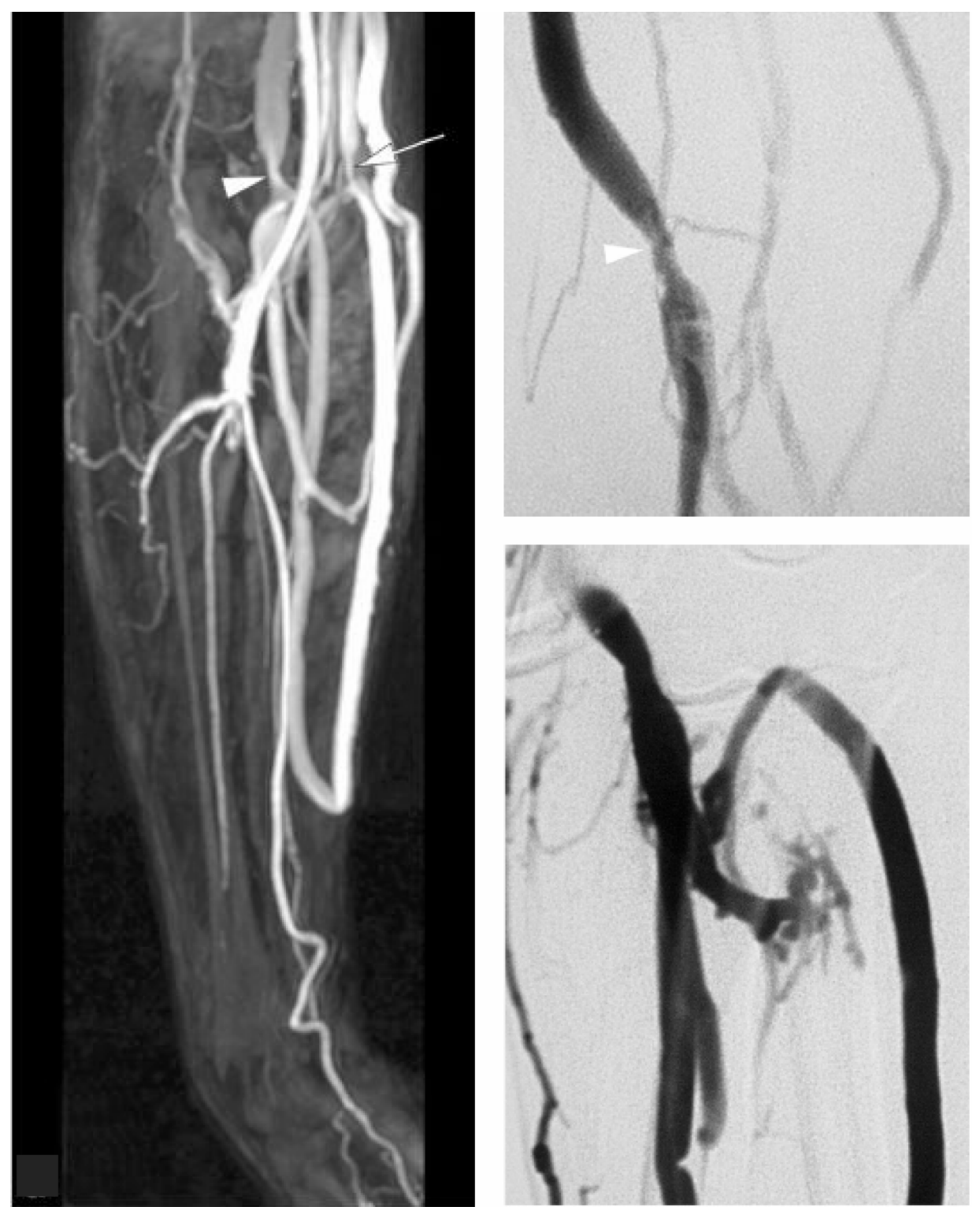

Figure 5: Whole-volume MIP of the second phase (11.0 seconds after start of injection of contrast medium) of a multiphase CE-MR angiogram of a PTFE loop graft AVF obtained in the left arm of a 57-year-old male patient (left). Stenoses are located at the venous (arrowhead) and arterial (arrow) anastomoses. Upper right: corresponding DSA of the venous anastomosis (arrowheads). Note that both techniques show similar degrees of stenosis. Lower right: corresponding DSA, showing retrograde filling of the arterial part of the loop graft AVF just distal to the arterial anastomosis. Note that the feeding artery is not opacified with DSA, and therefore the stenosis was missed. Primary PTA of the venous anastomosis did not induce flow recovery, and only after the arterial stenosis was dilated flow recovered. 

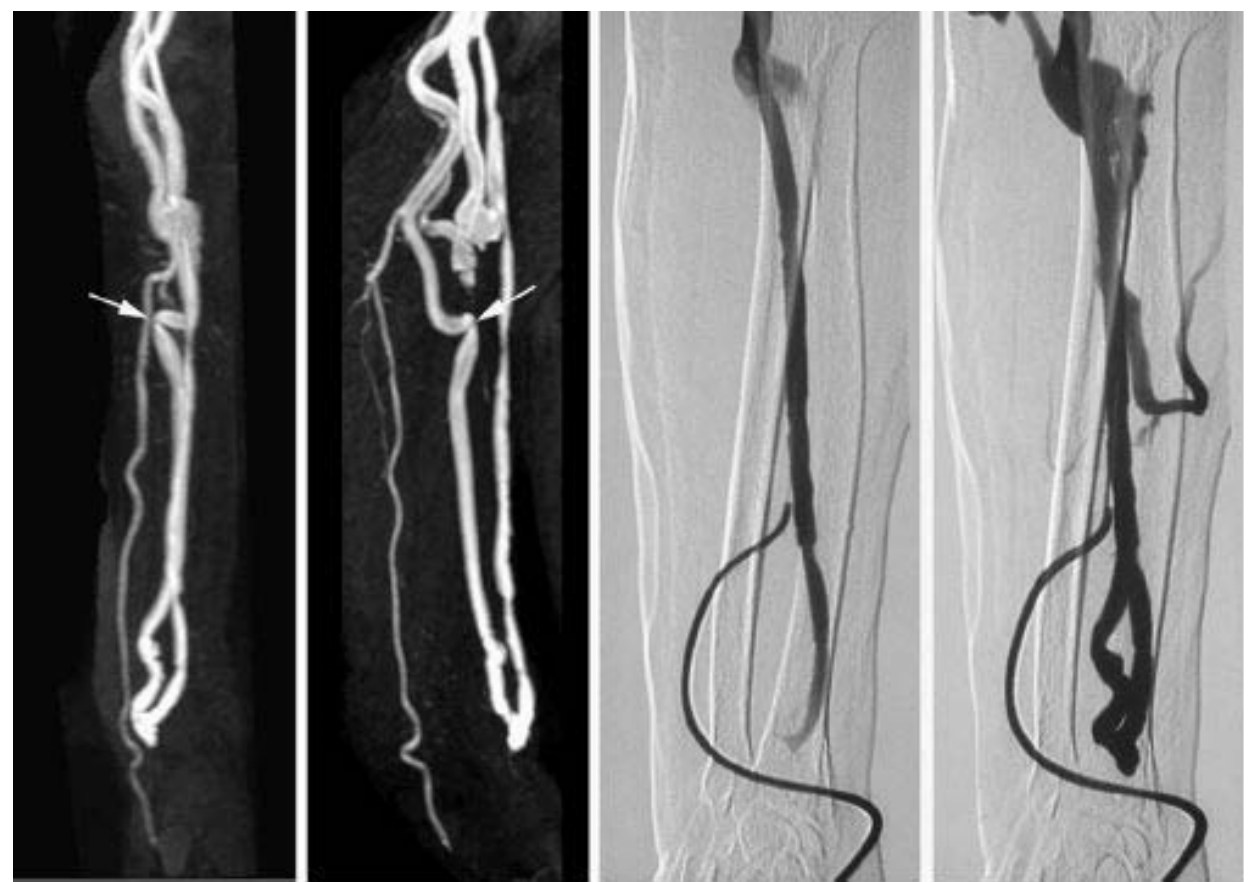

Figure 6: Whole-volume MIPs (left two panels) of the second phase (9.5 seconds after start of injection of contrast medium) of a multiphase CE-MR angiogram of a straight PTFE graft AVF obtained in the right arm of a 69-year-old female patient. The left panel is an oblique coronal view of the forearm arteries and the AVF and shows kinking and a high-grade stenosis in the feeding artery (arrow). The second panel shows a coronal view in which the kinking can be appreciated more clearly (arrow). The right two panels show the best-available DSA images of the AVF. Note that the kinking is not visible in these projections. 


\section{Chapter 8}

\section{Interobserver agreement}

The linear weighted kappa value regarding the differentiation between hemodynamically significant vs. nonsignificant stenoses was $0.81(95 \% \mathrm{Cl}=0.71-$ 0.92) for CE-MRA and $0.69(95 \% \mathrm{Cl}=0.55-0.84)$ for DSA, (Table 2) indicating almost perfect agreement on CE-MRA and substantial agreement on DSA (11).

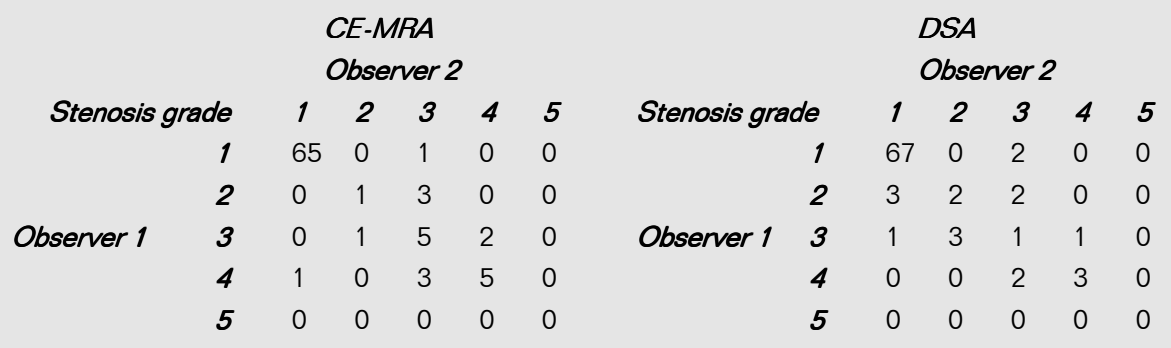

Table 2: Interobserer agreement for stenosis detection (analysis was done on segment level; in every cell is the number of vessel segments with the particular combination of test results on CE-MRA and DSA. Grade $1=0-20 \%$; 2 = 21-49\%; $3=50-74 \%$; $4=75-99 \%$; 5 = occlusion).

\section{Discussion}

With this preliminary study we have shown that high resolution multiphase CEMRA is a reliable and reproducible tool for the detection of flow-limiting stenoses in hemodialysis AVF. CE-MRA images of all AVF were considered by both observers to be of excellent diagnostic quality. Due to the use of a rectangular surface coil $(100 \times 400 \mathrm{~mm})$, the FOV was limited to this size. Therefore, no information was available about arteries and veins proximal to the cubital fossa, and this is the most important limitation of this study, because it is known that flowlimiting stenoses may be located in arteries and veins draining AVF (12). However, imaging with a larger FOV would have led to longer imaging times and would have made multiphase imaging more difficult. Another limitation of the current study is the relatively large number of false positive lesions detected on CE-MRA, which lowers specificity and PPV. Retrospective analysis of these lesions showed that most were borderline positive, whereas at DSA these lesions were borderline negative. The most likely reason for this finding is that with the resolution used in the current study, stenosis overestimation still cannot be avoided, as was also shown by Bos et al (5). False positive lesions were 
especially prevalent when $>50 \%$ was used as the threshold for classifying an AVF as diseased. Although no stenoses were missed at this threshold, observer 1 classified five and observer 2 classified four patients as having a stenosis $>50 \%$ on CE-MRA, whereas at DSA these patients did not have stenoses exceeding this threshold. At the $>75 \%$ threshold, these numbers were three and two patients for observers 1 and 2, respectively. In contrast to CE-MRA, not all DSA images of the separate vessel segments were scored of excellent diagnostic quality. In this respect, CE-MRA of AVF turned out to be a valuable technique. Due to filling of a distal cephalic vein branch, the anastomotic region was not well defined in two cases and two hemodynamic important stenoses were missed. In one patient, the stenosis was located in the feeding artery; in the other patient, the stenosis was located at the anastomosis. Analysis in the angiography suite of the available CE-MRA images prevented a second DSA session in these two patients. The PTA interventions based on the CE-MRA images turned out to be successful in terms of recovered flow in the first dialysis sessions afterwards. In three other patients, CE-MRA detected stenoses in vessel segments that were not depicted with DSA. Initially, other lesions were treated, but flow was recovered only after the lesions also seen on CE-MRA were treated (Fig. 5). An extreme example is a patient with a straight graft AVF (from distal radial to proximal cephalic vein), where multiple diagnostic and interventional DSA sessions prior to the current study did not induce flow recovery. Analysis of the CE-MRA images showed a severe kink in the proximal radial artery. This kink was missed by DSA due to vessel overlap (Fig. 6), and surgical graft interposition of the part of the artery with the kink based on the CE-MRA images led to flow recovery. The number of stenoses detected on CE-MRA and DSA images differed. Both observers detected no false negative stenoses, but 6 and 11 false positive stenoses. There are several explanations for this high number of false positive results. One explanation may be that DSA is less sensitive than CE-MRA due to the limited number of projections obtainable. Also, vein branches dilate because flow is interrupted with DSA, changing the anatomical situation (and thus possibly the grade of stensosis) compared to the non-flowinterrupted CEMRA images. Since all patients included in this study experienced a flow decline in their AVF, it can be assumed that there must be at least one flow-limiting stenosis in the AVF or in the vessels supplying or draining the AVF. What are the 
implications of the current study for clinical practice? Because failure to detect a significant stenosis may have far-reaching consequences for the patient (i.e., shunt thrombosis and having to operate the patient to make a new shunt (1)), sensitivity should be as high as possible, and the low specificity and NPV are of less concern in clinical practice. We feel that the current study has shown that multiphase CE-MRA of AVF is not so much a tool to determine the exact degree of a lesion as it is a particularly valuable technique to pinpoint the exact location of a stenosis, allowing for viewing from multiple angles and better able to visualize the arteries feeding the AVF. If a stenosis is found at DSA, it is often assumed that this is the culprit lesion, which is not always the case. In the current study, there were two cases in which additional stenoses, as seen on CE-MRA only, were the culprit lesions, as evidenced by increase in access flow rates after those stenoses were dilated. On the other hand, MRA techniques, including CE methods, are known to overestimate stenoses (5-9), and we cannot rule out that this was the case in the current study. However, we found DSA to be an imperfect standard of reference because of vessel overlap, inadequate retrograde filling of the AVF, and filling of vein branches, which complicate image analysis. From a practical and medical ethical perspective, DSA is the best standard of reference available at this moment. In general, CE-MRA has advantages over DSA. The patient and physicians are not exposed to ionizing radiation; the gadolinium containing contrast medium is not contraindicated for use in patients with impaired renal function and can be removed by hemodialysis (13-15). Another advantage of using CE-MRA for the evaluation of flow-limited AVF is that prior to an intervention, lesions can be identified also in the arteries supplying the AVF, making the DSA a more targeted procedure. Ideally, however, central vessels should also be depicted. The procedure presented in this study takes only 15-20 minutes and is less invasive than conventional DSA. In addition to functional anatomic images, flow rates can also be measured in the same session using PC velocity mapping (16). In the current study, this was not done because flow measurements were already obtained with ultrasound dilution technique.

In conclusion, CE-MRA can provide excellent visualization of forearm hemodialysis access AVF and is highly sensitive for the detection of flow-limiting stenoses. Using the current parameter settings, however, specificity is 
suboptimal, but this is clinically less relevant. CE-MRA is a useful diagnostic tool prior to conventional DSA to pinpoint culprit lesions in vessel segments not easily imaged with DSA. Further studies need to be conducted to find out if preinterventional CE-MRA can improve long-term AVF patency rates. 


\section{References}

1. Murphy GJ, White SA, Nicholson ML. Vascular access for haemodialysis. Br J Surg 2000; 87:1300-1315.

2. III. NKF-K/DOQI Clinical Practice Guidelines for Vascular Access: update 2000. Am J Kidney Dis $2001 ; 37:$ S137-181.

3. Staple TW. Retrograde venography of subcutaneous arteriovenous fistulas created surgically for hemodialysis. Radiology 1973; 106:223-224.

4. Waldman GJ, Pattynama PM, Chang PC, Verburgh C, Reiber JH, de Roos A. Magnetic resonance angiography of dialysis access shunts: initial results. Magn Reson Imaging 1996; 14:197-200.

5. Bos $\mathrm{C}$, Smits $\mathrm{JH}$, Zijlstra JJ, et al. MRA of hemodialysis access grafts and fistulae using selective contrast injection and flow interruption. Magn Reson Med 2001; 45:557-561.

6. Konermann M, Sanner B, Laufer U, Josephs W, Odenthal HJ, Horstmann E. Magnetic resonance angiography as a technique for the visualization of hemodialysis shunts. Nephron 1996; 73:73-78.

7. Gehl HB, Bohndorf K, Gladziwa U, Handt S, Gunther RW. Imaging of hemodialysis fistulas: limitations of MR angiography. J Comput Assist Tomogr 1991; 15:271-275.

8. Cavagna E, D'Andrea P, Schiavon F, Tarroni G. Failing hemodialysis arteriovenous fistula and percutaneous treatment: imaging with $\mathrm{CT}, \mathrm{MRI}$ and digital subtraction angiography. Cardiovasc Intervent Radiol 2000; 23:262-265.

9. Laissy JP, Menegazzo D, Debray MP, et al. Failing arteriovenous hemodialysis fistulas: assessment with magnetic resonance angiography. Invest Radiol 1999; 34:218-224.

10. van Erkel AR, Pattynama PM. Receiver operating characteristic (ROC) analysis: basic principles and applications in radiology. Eur J Radiol 1998; 27:88-94.

11. Cohen J. Weighted kappa: nominal scale agreement with provision for scale disagreement or partial credit. Psychol Bull 1968; 70:213-230.

12. Langeveld APM, Leunissen KML, Eikelboom BC, Kitslaar PJEHM, Tordoir JHM. Duplex ultrasound detection of stenoses in newly created hemodialysis AV fistula. Maastricht: Datawyse, 1990.

13. Runge VM. Safety of approved MR contrast media for intravenous injection. J Magn Reson Imaging 2000; 12:205-213.

14. Prince MR, Arnoldus C, Frisoli JK. Nephrotoxicity of high-dose gadolinium compared with iodinated contrast. J Magn Reson Imaging 1996; 6:162-166.

15. Choyke PL, Girton ME, Vaughan EM, Frank JA, Austin HA, 3rd. Clearance of gadolinium chelates by hemodialysis: an in vitro study. J Magn Reson Imaging 1995; 5:470-472.

16. Oudenhoven LF, Pattynama PM, de Roos A, Seeverens HJ, Rebergen SA, Chang PC. Magnetic resonance, a new method for measuring blood flow in hemodialysis fistulae. Kidney Int 1994; 45:884-889. 




\section{Chapter 9}

General discussion, future perspective and conclusion 


\section{General discussion}

End-stage renal disease (ESRD) leads to severe illness and death if left untreated. The number of patients requiring renal replacement therapy by hemodialysis is rising rapidly (1-3). A well functioning vascular access is a prerequisite for successful hemodialysis treatment. Achieving long-term hemodialysis vascular access is becoming a challenge because of an increase in elderly hemodialysis patients with various co-morbidities that are associated with poor and diseased vessels. This makes successful vascular access creation cumbersome (4-6). To reduce vascular access related complications, autogenous arteriovenous fistulas (AVF) are preferred over arteriovenous grafts (AVG) (4-6). However, the clinical success of AVF is jeopardized by high early failure and nonmaturation rates. Early failure and non-maturation necessitate salvage procedures and the use of indwelling central venous catheters (CVCs). These procedures are associated with increased morbidity and mortality (4-7). Guidelines recommend the use of diagnostic modalities, duplex ultrasound (DUS) in particular, to enable tailored vascular access creation for individual patients to avoid vascular access early failure and non-maturation (4-6). However, even with the use of current available diagnostic modalities in the workup of patients requiring a hemodialysis vascular access, reported early failure and nonmaturation rates are unacceptably high (8-17). The purpose of this thesis was to assess the clinical value of diagnostic modalities in the work-up prior to vascular access creation. Furthermore, the performance of contrast-enhanced magnetic resonance angiography (CE-MRA) was determined to detect stenoses in patients who are at risk for vascular access thrombosis.

\section{Preoperative assessment}

Chapter 2 reviewed currently available diagnostic modalities to assess the upper extremity vasculature prior to vascular access creation. For this purpose, DUS is the most widely used and recommended modality (4-6). However, developments in MRA technique and the availability of MR contrast media have increased interest in the clinical value of using CE-MRA prior to vascular access creation (18-24). Yet, thus far, the only data about the clinical value of CE-MRA to predict vascular access outcome are limited to those reported in chapter 6 and 7 of the present thesis. Furthermore, prior research has demonstrated that various 
preoperative parameters are associated with an increased risk of vascular access early failure and non-maturation. Patient characteristics such as age ( $>65$ years), peripheral arterial occlusive disease, coronary artery disease and race (dark skin color) are associated with an increased risk of vascular access early failure and non-maturation (25). In addition, preoperatively determined vessel characteristics such as large arterial (15, 26-30) and venous diameters (11, 15, 27-29, 31, 32), high arterial blood flow, low resistive indices, normal venous flow patterns and the absence of obstructions are associated with lower AVF early failure rates and lower AVF non-maturation rates $(26,31)$.

Vascular access guidelines have recommended the use of adequately sized vessels without obstructions to create a vascular access for hemodialysis to reduce the incidence of vascular access early failure and non-maturation (4-6). However, in these guidelines, no cut-off values of reported parameters (i.e., what is an adequate vessel diameter) have been established and no standardized DUS protocol has been suggested. To date, none of the preoperative parameters reported in literature enabled good prediction of successful AVF maturation at patient level. Furthermore, there are no data about measurement reproducibility of these parameters. This is important because the clinical value of these parameters is determined by their reproducibility.

\section{Forearm superficial venous diameter measurements}

Small forearm superficial venous diameters are associated with an increased risk of AVF early failure and non-maturation (4-6, 11, 15, 27-29, 31, 32). However, the exact cut-off value needs to be determined. Chapter 3 describes an experiment in which the reproducibility of forearm superficial venous diameter measurements was assessed in a group of 10 healthy male volunteers. This experiment sought to determine the effect of venous congestion on forearm superficial venous diameter and on cross sectional area size and shape. The relation between venous congestion pressure and diameter measurement reproducibility was also investigated. B-mode image analysis turned out to be largely observer independent. Reproducibility of repeated forearm superficial diameter measurements was best at venous congestion pressures $>40 \mathrm{mmHg}$. Furthermore, forearm superficial venous diameters substantially increased due to venous congestion pressure increase. The data also demonstrated that the cross 


\section{Chapter 9}

sectional area of forearm superficial veins was not circular but elliptical in shape, even at venous congestion pressures $>40 \mathrm{mmHg}$.

Chapter 4 describes another experiment to determine the reproducibility of forearm superficial venous diameter measurements, as assessed in a group of 21 end-stage renal disease patients. More specifically, the effect of observer, time (i.e. day to day variation) and venous congestion method on diameter measurements was investigated. Forearm superficial venous diameter measurements were independent of observer and of which congestion method was used. However, forearm superficial venous diameters showed substantial variation over time with variation coefficients of $26 \%$ and $27 \%$ when using a cuff or tourniquet, respectively. Forearm superficial venous diameter is thus affected by daily variation and the applied venous congestion pressure.

Other factors that have also been reported to affect venous diameters are temperature and patient positioning, as reported by van Bemmelen et al. (33). A single forearm superficial venous diameter seems therefore of limited, if any, value to predict vascular access function due to poor reproducibility. Furthermore, a comprehensive preoperative assessment should preferably also include assessment of arteries and cardiac function. The reproducibility of arterial measurements, however, was not a topic of this thesis and remains to be determined. Furthermore, serial measurements of multiple parameters of different vascular access components (e.g. heart, arteries, veins, capillaries) might result in better understanding of adaptations following vascular access creation. These changes and further improvements in preoperative assessment might result in better prediction and thereby anticipation of vascular access early failure and non-maturation.

\section{CE-MRA in the work-up prior to vascular access creation}

Chapter 5 describes a new CE-MRA protocol. The development of this protocol enabled selective depiction of arteries and veins of the entire upper extremity, thorax aperture and mediastinum with good to excellent image quality by scanning the upper extremity vasculature in two separate stations.

In chapter 6 a prospective study on the clinical value of this new CE-MRA protocol to predict vascular access maturation was compared to DUS. In this 
study the vascular access early failure and non-maturation rate was 30\%. Mostly, a pre-existing central venous obstruction detected by CE-MRA was the cause of early access failure. These central venous obstructions, however, were not detected by DUS. The incidence of non-maturation was highest among RC-AVFs (60\% of all RC-AVF) when compared to other vascular access types. RC-AVF non-maturation was associated with the presence of large caliber accessory veins or venous outflow stenoses.

As suggested by the above presented results, CE-MRA potentially better predictis vascular access outcome than DUS. CE-MRA correctly predicted early access failure or access non-maturation in 33\% (7/21) of the patients in whom a vascular access failed to mature. Because surgery for the patients included in this study was DUS guided, the CE-MRA treatment plans were hypothetical. Therefore the present results need to be confirmed in a prospective randomized clinical trial. The use of CE-MRA to detect arterial and venous stenosis as well as obstructions proved to be highly observer independent. An important additional value of CE-MRA in the work-up prior to vascular access creation lies in excellent assessment of central arterial and venous vessels, where DUS shows poor performance (34). However, CE-MRA has also some shortcomings. A substantial group of patients could not be assessed with MR imaging in our study because of pacemakers, claustrophobia or other MR imaging contra-indications. This shortcoming, might partially be resolved, though, as recent reports suggest that MR imaging is a safe procedure in patients with pacemakers or implantable cardioverter-defibrilators under controlled conditions by taking both MR- and device precautions (35-37).

Successful AVF maturation depends on both AVF diameter $(>4 \mathrm{~mm})$ and flow rate $(>500 \mathrm{~mL} / \mathrm{min})$ (38). A recent study from our group showed that large caliber accessory veins play an important role in AVF flow distribution, as determined by a lumped parameter model. An increase in accessory vein caliber results in an increase in accessory vein flow rate and thus in a subsequent decrease in AVF flow rate (39). In addition, Beathard et al. reported that ligation of accessory veins was a successful salvage procedure in a substantial group of patients in whom the AVF failed to mature (40). Thus accessory veins play an important role in AVF non-maturation. So far, however, there are no data about 
preoperatively detected large caliber accessory veins and their possible influence on clinical outcome.

In chapter 7, the preoperative presence of accessory veins and their relation to RC-AVF (non)-maturation was determined in a group of 15 consecutive patients. The data show that in these patients large caliber accessory veins as detected by CE-MRA or venous outflow stenoses were associated with RC-AVF nonmaturation. Furthermore, venous outflow stenoses were de novo stenosis in the group of patients receiving a RC-AVF because they were not detected preoperatively by either DUS or CE-MRA. Non-maturation due to 'de novo' venous outflow stenoses can not be anticipated, in contrast to non-maturation due to large caliber accessory veins. It remains elusive, however, if ligation of large caliber accessory veins during RC-AVF creation can avoid non-maturation in these patients.

\section{Detection of stenosis in dysfunctional vascular accesses}

After having achieved a functional vascular access, prevention of vascular access failure is essential. Interventions (e.g. surgical revision, percutaneous intervention) are important to prevent vacular access failure. Approximately 0.20.4 and 0.8-1.0 interventions are necessary per patient per year for AVFs and AVGs, respectively (41-43).

The main cause of vascular access failure is thrombosis caused by flow limiting stenoses. Patients at risk for access thrombosis are currently detected by taking monthly vascular access flow measurements during their hemodialysis treatment. A decrease in access flow or a change in intra-access pressure is indicative for stenosis development (4-6). Furthermore, a decrease in intra-access flow combined with intra-access pressure increase is indicative for a flow limiting venous outflow stenosis. In contrast, a decrease in both access flow and intraaccess pressure is indicative for a flow limiting arterial inflow stenosis (44).

Diagnostic imaging and pre-emptive interventions enable prevention of vascular access failure and thereby a decrease in vascular access related healthcare costs (45). Digital subtraction angiography (DSA) according to the method of Staple, the current standard of reference, is inconclusive in a substantial number of examinations because of insufficient retrograde arterial contrast media filling and 
vessel overlap. This leads to repeated DSAs and interventions that are inconvenient for patients and also costly $(46,47)$. The number of diagnostic DSA procedures should be kept to a minimum because they expose patients to a radiation dose and iodinated contrast media that may even further impair renal function. This may lead to an increase in morbidity and mortality (48-50). Taken together, additional diagnostics are needed in patients for whom DSA is inconclusive.

Chapter 8 describes a study in which the value of CE-MRA to detect flow limiting stenoses in AVFs and AVGs was assessed with DSA according to the method of Staple as standard of reference. The results demonstrate that CE-MRA was highly sensitive for detection of stenosis. CE-MRA, therefore, appears an attractive and valuable additional diagnostic modality to detect flow limiting stenoses in patients at risk of vascular access thrombosis. In contrast to CEMRA, arterial inflow obstructions were missed by DSA in a substantial number of the patients.

Since the results in chapter 8 have been published in 2003, several subsequent studies have been reported that compare DUS, CE-MRA and DSA to detect flow limiting stenoses in patients at risk for vascular access thrombosis (51-53). DUS proved to be a valuable diagnostic tool prior to DSA. It potentially prevents inconclusive DSAs. DUS enables better selection DSA puncture sites for contrast media injection (51). DUS also appears a valuable diagnostic modality to detect upper extremity venous outflow stenosis located from the wrist to the axilla (54). However to assess arterial inflow stenoses and stenosesin the anastomotic region as well as central arterial or central venous stenoses DUS is time consuming, observer dependent and shows poor performance $(34,44,54)$. Therefore, the application of DUS prior to DSA is a matter of discussion.

There are new DSA techniques such as retrograde catheterization of the arterial inflow that enable assessment of central arteries $(55,56)$. However, retrograde catheterization of the arterial inflow exposes patients to an increased risk of cerebral embolism and is frequently associated with silent ischaemic brain lesions (57). CE-MRA is the preferred technique to assess the central arteries because it is less invasive, does not require arterial catheterization nor injection of nephrotoxic contrast media and is furthermore not accompanied by radiation 
exposure (46). Another advantage of CE-MRA compared to DSA and DUS is the 3-D nature of the CE-MRA data and it enables better insight into the relation of the vascular structures with surrounding tissues $(46,51-53)$.

In summary, CE-MRA provides excellent visualization of hemodialysis accesses that are at risk of access thrombosis. It is also highly sensitive to detect flowlimiting stenoses. Furthermore, CE-MRA is a useful pre-interventional diagnostic tool, especially to pinpoint culprit lesions in vessel segments that are not easily imaged with DSA. However, further studies need to be conducted to determine whether pre-interventional CE-MRA can improve long-term AVF patency rates and to determine if pre-interventional CE-MRA is cost-effective.

\section{Future perspective}

\section{Preoperative assessment}

Vascular access related complications place a large burden on healthcare facilities, manpower and on costs. Therefore, tailored vascular access for individual patients is important to reduce the incidence of early failure, nonmaturation and long-term complications.

Recommendations for preoperative assessment in vascular access guidelines need to be completed in detail with special attention to assessment protocols. Furthermore, all parameters used that are to determine vascular access surgery strategies need to be further explored by assessing measurement reproducibility and their causal relation with access maturation. As concluded from chapter 3, 4 and 5, forearm superficial venous diameter is susceptible to changes in assessment protocol and shows variation over time. Therefore, a single diameter measurement does not serve the purpose of a parameter to successfully determine the treatment plan on patient level. Other research groups suggest the use of dynamic instead of static parameters to characterize a vessel $(4,31,58$, 59). However, because of the poor reproducibility of venous diameter measurements and especially of diameter measurements at low venous congestion pressure, measurement reproducibility of venous compliance or distensibility as a single parameter is inadequate and, therefore, not suitable for clinical use. To better predict vascular access outcome a combination of arterial, venous and cardiac parameters should be used instead of a single parameter. It is 
essential to optimize, validate and standardize pre-operative diagnostic modalities (i.e., DUS and CE-MRA) and parameters (i.e., diameter, compliance, distensibility, flow, resistance index etc.) that are used in the work-up prior to vascular access creation. This should be the challenge for scientists in vascular access related research in the near future, to avoid the use of specific preoperative parameters to determine the type of access needed for individual patients without the use of validated, standardazed protocols.

Early failure and non-maturation are different entities occurring in different types of vascular accesses due to different causes. Adaptations after vascular access creation and mechanisms responsible for AVF maturation are poorly understood. From chapter 6 and 7, it can be concluded that early failure is associated with pre-existing venous outflow stenoses and occlusions whereas non-maturation is associated with large calibre accessory veins and 'de novo' venous outflow stenosis that develop after access creation. Preoperative vessel assessment by CE-MRA enables better prediction of vascular access function at patient level than DUS because it enables excellent assessment of central arteries and veins as well as identification and location of accessory veins. Therefor, clinical application of CE-MRA enables treatment of culprit lesions associated with poor clinical outcome prior to vascular access creation and thereby prevention of vascular access early failure and non-maturation. Preoperative detection of large calibre accessory veins also enables ligation of these accessory veins and thereby prevention of RC-AVF non-maturation. Non-maturation due to 'de novo' stenosis, however, can not be anticipated and requires postoperative monitoring and treatment.

A better understanding of adaptations following vascular access creation is essential to better prediction of vascular access outcome on a patient level. More insight in preoperative and postoperative vascular geometry, topology and local vessel wall properties as well as their effect on local and systemic hemodynamics is needed to better understand and predict adaptations following vascular access creation. Computer simulations using a patient specific lumped parameter model enables simulation of vascular access creation $(39,60)$. Such a model consists of serially connected segments that can be made patient specific using preoperative patient measurements (CE-MRA and DUS) for all input data. Ultimately such a model can be used clinically to predict vascular access outcome on patient level 


\section{Chapter 9}

or to determine which parameter or combination of parameters is essential to successfully predict vascular access early failure and non-maturation.

\section{Detection of stenoses in dysfunctional vascular accesses}

Preinterventional CE-MRA is highly sensitive to detect culprit lesions and can reduce inconclusive DSA procedures. CE-MRA, when available, is the first choice imaging modality because of its high sensitivity and the avoidance of retrograde catherization during DSA. Future research, however, needs to determine if clinical application of preinterventional CE-MRA is cost effective as a first choice imaging modality for detection of stenoses in dysfunctional vascular accesses.

Further developments in intra-access pressure and flow measurement techniques for access surveillance purposes during hemodialysis treatment might enable better localization of developing stenoses and thereby help the interventional radiologist to avoid inconclusive DSA procedures. Pressure wave analysis, for example, potentially enables early detection and localization of developing stenoses during hemodialysis treatment by detection of changes in mean pressure and pressure wave amplitude. It would be interesting to determine the clinical value of intra-access pressure wave analysis in the near future. 


\section{Conclusions of this thesis}

1. Preoperative DUS assessment of vessel quality and diameter does not result in low vascular access early failure - and non-maturation rates.

2. The reproducibility of forearm superficial venous diameter measurements strongly depends on the applied venous congestion pressure. In addition there is substantial daily variation in forearm superficial venous diameter.

3. CE-MRA enables high fidelity depiction of the entire upper extremity vasculature prior to vascular access creation.

4. CE-MRA is a valuable diagnostic tool prior to vascular access creation that enables accurate identification and location of culprit lesions associated with vascular access early failure and non-maturation.

5. Preoperative identification of large caliber accessory veins will play an important role in future prevention of RC-AVF non-maturation.

6. CE-MRA is a highly accurate imaging modality for the preinterventional identification and location of culprit lesions in hemodialysis vascular accesses at risk of thrombosis. 


\section{References}

1. Grassmann A, Gioberge S, Moeller S, Brown G. ESRD patients in 2004: global overview of patient numbers, treatment modalities and associated trends. Nephrol Dial Transplant 2005; 20:2587-2593.

2. Meichelboeck W. ESRD 2005 - A Worldwide Overview - Facts, Figures and Trends. In: Mickley V, ed. 4th International Congress of the Vascular Access Society (VAS). Berlin, Germany: Blood Purification, Karger, 2005; 227-261.

3. Moeller S, Gioberge S, Brown G. ESRD patients in 2001: global overview of patients, treatment modalities and development trends. Nephrol Dial Transplant 2002; 17:2071-2076.

4. Tordoir JH, Mickley V. European guidelines for vascular access: clinical algorithms on vascular access for haemodialysis. Edtna Erca J 2003; 29:131-136.

5. III. NKF-K/DOQI Clinical Practice Guidelines for Vascular Access: update 2000. Am J Kidney Dis $2001 ; 37: \mathrm{S} 137-181$.

6. Clinical practice guidelines for vascular access. Am J Kidney Dis 2006; 48 Suppl 1:S176-247.

7. Rayner HC, Pisoni RL, Bommer J, et al. Mortality and hospitalization in haemodialysis patients in five European countries: results from the Dialysis Outcomes and Practice Patterns Study (DOPPS). Nephrol Dial Transplant 2004; 19:108-120.

8. Huber TS, Carter JW, Carter RL, Seeger JM. Patency of autogenous and polytetrafluoroethylene upper extremity arteriovenous hemodialysis accesses: a systematic review. J Vasc Surg 2003; 38:1005-1011.

9. Allon M, Robbin ML. Increasing arteriovenous fistulas in hemodialysis patients: problems and solutions. Kidney Int 2002; 62:1109-1124.

10. Allon M, Lockhart ME, Lilly RZ, et al. Effect of preoperative sonographic mapping on vascular access outcomes in hemodialysis patients. Kidney Int 2001; 60:2013-2020.

11. Ascher E, Gade P, Hingorani A, et al. Changes in the practice of angioaccess surgery: impact of dialysis outcome and quality initiative recommendations. J Vasc Surg 2000; 31:84-92.

12. Miller PE, Tolwani A, Luscy CP, et al. Predictors of adequacy of arteriovenous fistulas in hemodialysis patients. Kidney Int 1999; 56:275-280.

13. Mihmanli I, Besirli K, Kurugoglu S, et al. Cephalic vein and hemodialysis fistula: surgeon's observation versus color Doppler ultrasonographic findings. J Ultrasound Med 2001; 20:217222.

14. Patel ST, Hughes J, Mills JL, Sr. Failure of arteriovenous fistula maturation: an unintended consequence of exceeding dialysis outcome quality Initiative guidelines for hemodialysis access. J Vasc Surg 2003; 38:439-445; discussion 445.

15. Robbin ML, Gallichio MH, Deierhoi MH, Young CJ, Weber TM, Allon M. US vascular mapping before hemodialysis access placement. Radiology 2000; 217:83-88. 
16. Tordoir JH, Rooyens P, Dammers R, van der Sande FM, de Haan M, Yo TI. Prospective evaluation of failure modes in autogenous radiocephalic wrist access for haemodialysis. Nephrol Dial Transplant 2003; 18:378-383.

17. Rooijens PP, Tordoir JH, Stijnen T, Burgmans JP, Smet de AA, Yo TI. Radiocephalic wrist arteriovenous fistula for hemodialysis: meta-analysis indicates a high primary failure rate. Eur $\mathrm{J}$ Vasc Endovasc Surg 2004; 28:583-589.

18. Planken RN, Tordoir JHM, de Haan MW, Backes WH, van Engelshoven JMA, Leiner T. Contrast Enhanced-MR Angiography of Upper Extremity Arteries and Veins Prior to Vascular Access Surgery. In: Mickley V, ed. 4th International Congress of the Vascular Access Society (VAS). Berlin: Karger, 2005; 227-261.

19. Meaney JF. Magnetic resonance angiography of the peripheral arteries: current status. Eur Radiol 2003; 13:836-852.

20. Wentz KU, Frohlich JM, von Weymarn C, Patak MA, Jenelten R, Zollikofer CL. High-resolution magnetic resonance angiography of hands with timed arterial compression (tac-MRA). Lancet 2003; 361:49-50.

21. Maki JH, Chenevert TL, Prince MR. Three-dimensional contrast-enhanced MR angiography. Top Magn Reson Imaging 1996; 8:322-344.

22. Weiger M, Pruessmann KP, Kassner A, et al. Contrast-enhanced 3D MRA using SENSE. J Magn Reson Imaging 2000; 12:671-677.

23. Ruehm SG, Zimny K, Debatin JF. Direct contrast-enhanced 3D MR venography. Eur Radiol 2001; 11:102-112.

24. Ruehm SG. MR venography. Eur Radiol 2003; 13:229-230.

25. Lok CE, Allon M, Moist L, Oliver MJ, Shah H, Zimmerman D. Risk Equation Determining Unsuccessful Cannulation Events and Failure to Mature in Arteriovenous Fistulas (REDUCE FTM I). J Am Soc Nephrol 2006.

26. Malovrh M. Non-invasive evaluation of vessels by duplex sonography prior to construction of arteriovenous fistulas for haemodialysis. Nephrol Dial Transplant 1998; 13:125-129.

27. Wong V, Ward R, Taylor J, Selvakumar S, How TV, Bakran A. Factors associated with early failure of arteriovenous fistulae for haemodialysis access. Eur J Vasc Endovasc Surg 1996; 12:207-213.

28. Silva MB, Jr., Hobson RW, 2nd, Pappas PJ, et al. A strategy for increasing use of autogenous hemodialysis access procedures: impact of preoperative noninvasive evaluation. J Vasc Surg 1998; 27:302-307; discussion 307-308.

29. Mendes RR, Farber MA, Marston WA, Dinwiddie LC, Keagy BA, Burnham SJ. Prediction of wrist arteriovenous fistula maturation with preoperative vein mapping with ultrasonography. $J$ Vasc Surg 2002; 36:460-463.

30. Lemson MS, Leunissen KM, Tordoir JH. Does pre-operative duplex examination improve patency rates of Brescia-Cimino fistulas? Nephrol Dial Transplant 1998; 13:1360-1361. 


\section{Chapter 9}

31. Malovrh M. Native arteriovenous fistula: preoperative evaluation. Am J Kidney Dis 2002; 39:1218-1225.

32. Brimble KS, Rabbat Ch G, Treleaven DJ, Ingram AJ. Utility of ultrasonographic venous assessment prior to forearm arteriovenous fistula creation. Clin Nephrol 2002; 58:122-127.

33. van Bemmelen PS, Kelly P, Blebea J. Improvement in the visualization of superficial arm veins being evaluated for access and bypass. J Vasc Surg 2005; 42:957-962.

34. Nack TL, Needleman L. Comparison of Duplex Ultrasound and Contrast Venography for Evaluation of Upper Extremity Venous Disease. The Journal Of Vascular Technology 1992; 16:69-73.

35. Faris OP, Shein M. Food and Drug Administration perspective: Magnetic resonance imaging of pacemaker and implantable cardioverter-defibrillator patients. Circulation 2006; 114:12321233.

36. Nazarian S, Roguin A, Zviman MM, et al. Clinical utility and safety of a protocol for noncardiac and cardiac magnetic resonance imaging of patients with permanent pacemakers and implantable-cardioverter defibrillators at 1.5 tesla. Circulation 2006; 114:1277-1284.

37. Sommer T, Naehle CP, Yang A, et al. Strategy for safe performance of extrathoracic magnetic resonance imaging at 1.5 tesla in the presence of cardiac pacemakers in non-pacemakerdependent patients: a prospective study with 115 examinations. Circulation 2006; 114:12851292.

38. Robbin ML, Chamberlain NE, Lockhart ME, et al. Hemodialysis arteriovenous fistula maturity: US evaluation. Radiology 2002; 225:59-64.

39. Vosse van der FN, Planken RN, Huberts W, Bosboom EMH, Keuter XHA, Tordoir JHM. Accessory veins and native arteriovenous fistula non-maturation: to clip or not to clip that is the question. In. Munich: Elsevier Ltd., 2006.

40. Beathard GA, Arnold P, Jackson J, Litchfield T. Aggressive treatment of early fistula failure. Kidney Int 2003; 64:1487-1494.

41. Staramos DN, Lazarides MK, Tzilalis VD, Ekonomou CS, Simopoulos CE, Dayantas JN. Patency of autologous and prosthetic arteriovenous fistulas in elderly patients. Eur J Surg 2000; 166:777-781.

42. Kherlakian GM, Roedersheimer LR, Arbaugh JJ, Newmark KJ, King LR. Comparison of autogenous fistula versus expanded polytetrafluoroethylene graft fistula for angioaccess in hemodialysis. Am J Surg 1986; 152:238-243.

43. Leapman SB, Boyle M, Pescovitz MD, Milgrom ML, Jindal RM, Filo RS. The arteriovenous fistula for hemodialysis access: gold standard or archaic relic? Am Surg 1996; 62:652-656; discussion 656-657.

44. Surlan $\mathrm{M}$, Popovic $\mathrm{P}$. The role of interventional radiology in management of patients with endstage renal disease. Eur J Radiol 2003; 46:96-114.

45. Wijnen E, Planken N, Keuter X, et al. Impact of a quality improvement programme based on vascular access flow monitoring on costs, access occlusion and access failure. Nephrol Dial Transplant 2006;: 21(12): 3514-9. 
46. Planken RN, Tordoir JH, Dammers $\mathrm{R}$, et al. Stenosis detection in forearm hemodialysis arteriovenous fistulae by multiphase contrast-enhanced magnetic resonance angiography: preliminary experience. J Magn Reson Imaging 2003; 17:54-64.

47. Staple TW. Retrograde venography of subcutaneous arteriovenous fistulas created surgically for hemodialysis. Radiology 1973; 106:223-224.

48. Jansen MA, Hart AA, Korevaar JC, Dekker FW, Boeschoten EW, Krediet RT. Predictors of the rate of decline of residual renal function in incident dialysis patients. Kidney Int 2002; 62:10461053.

49. Geoffroy O, Tassart M, Le Blanche AF, et al. Upper extremity digital subtraction venography with gadoterate meglumine before fistula creation for hemodialysis. Kidney Int 2001; 59:14911497.

50. Merkus MP, Jager KJ, Dekker FW, de Haan RJ, Boeschoten EW, Krediet RT. Predictors of poor outcome in chronic dialysis patients: The Netherlands Cooperative Study on the Adequacy of Dialysis. The NECOSAD Study Group. Am J Kidney Dis 2000; 35:69-79.

51. Doelman C, Duijm LE, Liem YS, et al. Stenosis detection in failing hemodialysis access fistulas and grafts: Comparison of color Doppler ultrasonography, contrast-enhanced magnetic resonance angiography, and digital subtraction angiography. J Vasc Surg 2005; 42:739-746.

52. Froger CL, Duijm LE, Liem YS, et al. Stenosis detection with MR angiography and digital subtraction angiography in dysfunctional hemodialysis access fistulas and grafts. Radiology 2005; 234:284-291.

53. Han KM, Duijm LE, Thelissen GR, et al. Failing hemodialysis access grafts: evaluation of complete vascular tree with $3 \mathrm{D}$ contrast-enhanced MR angiography with high spatial resolution: initial results in 10 patients. Radiology 2003; 227:601-605.

54. Tordoir JH, de Bruin HG, Hoeneveld H, Eikelboom BC, Kitslaar PJ. Duplex ultrasound scanning in the assessment of arteriovenous fistulas created for hemodialysis access: comparison with digital subtraction angiography. J Vasc Surg 1989; 10:122-128.

55. Khan FA, Vesely TM. Arterial problems associated with dysfunctional hemodialysis grafts: evaluation of patients at high risk for arterial disease. J Vasc Interv Radiol 2002; 13:1109-1114.

56. Duijm LE, Liem YS, van der Rijt RH, et al. Inflow stenoses in dysfunctional hemodialysis access fistulae and grafts. Am J Kidney Dis 2006; 48:98-105.

57. Omran $H$, Schmidt $H$, Hackenbroch $M$, et al. Silent and apparent cerebral embolism after retrograde catheterisation of the aortic valve in valvular stenosis: a prospective, randomised study. Lancet 2003; 361:1241-1246.

58. van der Linden J, Lameris TW, van den Meiracker AH, de Smet AA, Blankestijn PJ, van den Dorpel MA. Forearm venous distensibility predicts successful arteriovenous fistula. Am J Kidney Dis 2006; 47:1013-1019.

59. van der Linden J, van den Meijracker AH, Vroegindeweij D, De Smet AEA, Lamersi TW, van den Dorpel MA. Forearm Blood Flow Reserve Predicts Successful Maturation of AV-Fistulae. J Am Soc Nephrol 2004; 14. 
Chapter 9

60. Westerhof N, Bosman F, De Vries CJ, Noordergraaf A. Analog studies of the human systemic arterial tree. J Biomech 1969; 2:121-143. 




\section{Chapter 10}

Samenvatting 


\section{Samenvatting}

Nierfunctieverlies kan leiden tot terminaal nierfalen. Het noodzakelijke gevolg is meestal niertransplantatie of nierfunctie vervangende therapie, zoals buikvliesspoeling of hemodialyse. Door een tekort aan donororganen is de meerderheid van deze patiëntengroep afhankelijk van nierfunctie vervangende therapie, met name hemodialyse.

Voor hemodialyse is een adequate vaattoegang vereist om het lichaam aan te sluiten op de kunstnier. Helaas veroorzaken vaattoegang gerelateerde complicaties zoals bijvoorbeeld trombose, infecties en ischemie van de hand een hoge morbiditeit, mortaliteit en hoge kosten voor de gezondheidszorg.

Doorgaans worden drie typen vaattoegang gebruikt voor hemodialyse. Deze zijn de autogene, met eigen vaten gemaakte, arterioveneuze fistel (AVF), de arterioveneuze graft (AVG) en de centraal veneuze catheter (CVC). De AVF wordt beschouwd als meest geschikte vaattoegang omdat deze langer bruikbaar is met minder complicaties in vergelijking met de AVG en CVC. Een AVF geeft aanleiding tot locale en systemische hemodynamische veranderingen. Het is namelijk een rechtstreekse verbinding tussen arterie en vene. Het capillaire vaatbed wordt gepasseerd wat een kortsluiting tussen het arteriële (hoge druk) en veneuze (lage druk) systeem veroorzaakt. Deze kortsluiting zorgt voor een toename in hartminuutvolume en bloedstroom toename door de AVF. Doordat de bloedstroom en intra-veneuze druk in de AVF toenemen, neemt ook de veneuze diameter toe. Toename in bloedstroom en diameter zijn van groot belang voor het successvol rijpen van de AVF. Het verwijden van de venen zorgt er namelijk voor dat deze beter aanprikbaar worden. De toename in bloedstroom is verder belangrijk om voldoende bloed door de kunstnier te laten stromen.

Naast de voordelen van de AVF ten opzichte van de andere typen vaattoegang, heeft de $A V F$ een aantal belangrijke beperkingen op korte termijn. Een substantieel aantal AVF's is na aanleg ongeschikt voor hemodialyse door het uitblijven van diameter- en/of bloedstroomtoename. Dit wordt non-maturatie genoemd. Uit eerder onderzoek is gebleken dat de kans op maturatie afhankelijk is van patiëntkarakteristieken zoals hart- en vaatziekten, leeftijd, geslacht en de kwaliteit van de te gebruiken arteriën en venen. Kleine, vernauwde of geobstrueerde vaten evenals de aanwezigheid van grote veneuze vertakkingen 
zijn geassocieerd met een verhoogde kans op non-maturatie.Ondanks het gebruik van ogenschijnlijk goede bloedvaten blijft maturatie toch uit in $20-50 \%$ van de gevallen.

In dit proefschrift is de klinische waarde van diagnostische modaliteiten en parameters ter voorkoming van non-maturatie van AVF's door middel van preoperatieve vaatonderzoeken onderzocht en beschreven. Dit is gedaan aan de hand van preoperatieve vaatonderzoeken met behulp van duplex ultrageluid (DUS) en contrast-versterkte magnetische resonantie angiografie (CV-MRA). Tevens is de klinische waarde bepaald van diagnostische modaliteiten om vernauwingen in hemodialyse vaattoegangen op te sporen.

In hoofdstuk 2 wordt een overzicht van de literatuur gepresenteerd over de status van diverse diagnostische modaliteiten voor het onderzoek van de armvaten voor de aanleg van een hemodialyse vaattoegang. Amerikaanse (NKF K-DOQI) en Europese (European vascular access society) richtlijnen bevelen het gebruik van DUS voor het preoperatieve onderzoek van de vaten aan. Met behulp van DUS kunnen diverse parameters worden gemeten om te bepalen voor welk type vaattoegang een patiënt in aanmerking komt. Tot op heden blijkt het echter onmogelijk om AVF non-maturatie te voorkomen. Het gebruik van preoperatieve diagnostiek zoals DUS met specifieke selectie criteria blijkt dus onvoldoende toereikend om non-maturatie te verminderen. Het is nog onduidelijk wat de reproduceerbaarheid van diverse parameter bepalingen is en of verbetering en standaardisering van onderzoeksprotocollen een daling kan bewerkstelligen in het aantal AVF non-maturaties.

In hoofdstuk 3 wordt in een groep van 10 gezonde mannelijke proefpersonen de reproduceerbaarheid van de diameter bepaling met DUS van oppervlakkige venen in de onderarm onderzocht. Daarnaast werd het effect van onderzoeker en de mate van veneuze stuwing op de diameter bepaald. De interpretatie van Bmode DUS beelden bleek onderzoeker onafhankelijk. De reproduceerbaarheid van de veneuze diameter bepaling was het beste bij een veneuze stuwingsdruk $>40 \mathrm{mmHg}$. Verder nam de diameter van oppervlakkige venen in de onderarm toe bij toename in veneuze stuwingsdruk. In tegenstelling tot eerder onderzoek bleek de transversale oppervlakte van de onderarmsvenen niet cirkelvormig maar ellipsvormig te zijn, ook bij stuwingsdrukken $>40 \mathrm{mmHg}$. Ten einde de 
onderarmsvenen beter te karakteriseren is bepaling van zowel de maximale als de minimale diameter bij een vastgestelde stuwingsdruk noodzakelijk.

In hoofdstuk 4 wordt in een groep van 21 patiënten met een terminale nierfunctiestoornis de reproduceerbaarheid van de diameterbepaling van oppervlakkige venen in de onderarm onderzocht met behulp van DUS. Deze studie onderzocht het effect van factoren als onderzoeker, variatie tussen twee verschillende onderzoekdagen en de methode van veneuze stuwing (drukmanchet of stuwband) op de reproduceerbaarheid van de diameter bepalingen. De diameterbepaling van oppervlakkige venen in de onderarm bleek niet afhankelijk van de beoordelaar of de gebruikte veneuze stuwingstechniek. De diameter bleek echter wel te variëren tussen de twee onderzoeksdagen met variantiecoefficiënten van $26 \%$ en $27 \%$ wanneer respectievelijk een manchet of stuwband werden gebruikt. De dagelijkse variantie van de veneuze diameter heeft dus belangrijke gevolgen voor het gebruik van veneuze diameters bij het maken van een keuze voor een type vaattoegang per patiënt.

In hoofstuk 5 wordt het nieuwe CV-MRA protocol onderzocht voor de afbeelding van arm-vaten. Met dit scan-protocol, waarmee de bloedvaten van de arm in twee stations worden afgebeeld, bleek het mogelijk om arteriën en venen van de gehele arm in één sessie af te beelden met zeer goede beeldkwaliteit.

In hoofdstuk 6 wordt in een prospectieve klinische studie de klinische waarde van CV-MRA voor het voorspellen van de vaattoegangfunctie vergeleken met het standaard DUS onderzoek. Het detecteren van arteriële en veneuze vernauwingen met behulp van CV-MRA bleek onderzoeker onafhankelijk. CVMRA bleek ook een waardevolle modaliteit en een betere voorspeller van de vaattoegang functie dan DUS.

In eerdere onderzoeken wordt beschreven dat grote veneuze zijtakken een rol zouden kunnen spelen in het al dan niet succesvol matureren van AVF's. Een mogelijke verklaring voor deze associatie is dat een grote veneuze zijtak de bloedtoevoer naar de AVF en de intraveneuze druk in de AVF doet afnemen waardoor maturatie uitblijft. In hoofdstuk 7 wordt de voorspellende waarde bepaald van de preoperatieve aanwezigheid van grote veneuze zijtakken op maturatie en non-maturatie. Preoperatief aangetoonde grote veneuze zijtakken bleken te zijn geassocieerd met non-maturatie van pols AVF's. Onderbinden van 
deze zijtakken resulteerde alsnog in maturatie. Het direct onderbinden van grote veneuze zijtakken zou daarom de incidentie van non-maturatie kunnen reduceren.

In hoofdstuk 8 wordt de klinische waarde van CV-MRA voor het detecteren van vernauwingen in reeds aangelegde AVF's en AVG's onderzocht. Deze techniek wordt vergeleken met digitale subtractie angiografie (DSA) als gouden standaard. Detectie van vernauwingen met CV-MRA resulteerde in een zeer hoge sensitiviteit en lage specificiteit. De lage specificiteit viel deels te wijten aan vernauwingen die werden gemist door DSA. Het merendeel van deze vernauwingen bleek achteraf klinisch relevant te zijn. Op basis van deze bevindingen wordt geconcludeerd dat CV-MRA een aantrekkelijke en waardevolle additionele diagnostische modaliteit is om vaattoegang vernauwingen te detecteren.

In hoofstuk 9 worden de bevindingen in dit proefschrift in perspectief geplaatst van de huidige literatuur. Ook worden de belangrijkste punten uit het proefschrift besproken. Het hoofdstuk wordt afgesloten met een discussie over mogelijke toekomstige ontwikkelingen. 


\section{Conclusies uit dit proefschrift}

1. Het gebruik van duplex ultrageluid onderzoek om preoperatief de kwaliteit en het kaliber van armvaten te bepalen om het juiste type vaattoegang voor de individuele patiënt te selecteren resulteert niet in verlaging van het percentage falende vaattoegangen tot een acceptabel niveau.

2. De reproduceerbaarheid van diameterbepalingen van oppervlakkige armvenen is afhankelijk van de daarbij toegepaste veneuze stuwingsdruk. Daarnaast vertoont de diameter van oppervlakkige armvenen aanzienlijke natuurlijke variatie in de tijd.

3. Met preoperatieve CV-MRA kunnen de arteriën en venen van de gehele bovenste extremiteit met grote precisie worden afgebeeld ten behoeve van de aanleg van een hemodialyse vaattoegang.

4. CV-MRA is een waardevolle techniek waarmee afwijkingen die de functie van de toekomstige hemodialyse vaattoegang beperken goed kunnen worden opgespoord.

5. Preoperatieve identificatie van grote accessoire venen zal een belangrijke rol spelen in de preventie van RC-AVF non-maturatie.

6. CV-MRA is een waardevolle onderzoekmethode voor het detecteren en localiseren van vernauwingen voorafgaand aan interventies in patiënten met een verhoogd risico op trombosering van hun hemodialyse vaattoegang. 


Curriculum vitae 


\section{Curriculum vitae}

Robrecht Nils Planken, was born on February 17, 1978 in The Hague in The Netherlands. He attended the 'Stichtse Vrije School' in Zeist and he finished his secondary school at 'Katholieke Scholengemeenschap De Breul' also in Zeist. Immediately thereafter, he started medical school at Maastricht University. During his studies he was president of the $4^{\text {th }}$ international Maastricht Medical Students Research Conference (1999/2000). Furthermore, he participated in research alongside his medical studies at the departments of gynacology \& obstetrics (1999/2000), surgery (2000/2003) and radiology (2002/2003). He started his medical internschips after obtaining his MSc degree in 2001 and received his MD degree in 2003. Thereafter, he started his $\mathrm{PhD}$ research project entitled "Hemodialysis vascular access imaging: duplex ultrasound and contrastenhanced magnetic resonance angiography" at the department of surgery at the Maastricht University Hopsital. He has presented original research and invited papers at many international meetings and has been awarded with the best poster presentation prize at the 100 year anniversary conference of the Dutch Society of Surgery and has been awarded with the first prize during the Pfizer Young Cardiovascular Grant Contest 2006 in 'Kasteel Oud Poelgeest' in Oegstgeest. Currently, he has an appointment as a resident at the department of surgery at St. Antonius Hospital Nieuwegein. 